\title{
CRITICAL MEASURES FOR VECTOR ENERGY: ASYMPTOTICS OF NON-DIAGONAL MULTIPLE ORTHOGONAL POLYNOMIALS FOR A CUBIC WEIGHT
}

\author{
ANDREI MARTÍNEZ-FINKELSHTEIN AND GUILHERME L. F. SILVA
}

AbStract. We consider the type I multiple orthogonal polynomials (MOPs) $\left(A_{n, m}, B_{n, m}\right), \operatorname{deg} A_{n, m} \leq n-1, \operatorname{deg} B_{n, m} \leq m-1$, and type II MOPs $P_{n, m}$, $\operatorname{deg} P_{n, m}=n+m$, satisfying non-hermitian orthogonality with respect to the weight $e^{-z^{3}}$ on two unbounded contours $\gamma_{1}$ and $\gamma_{2}$ on $\mathbb{C}$, with (in the case of type II MOPs) $n$ conditions on $\gamma_{1}$ and $m$ on $\gamma_{2}$. Under the assumption that

$$
n, m \rightarrow \infty, \quad \frac{n}{n+m} \rightarrow \alpha \in(0,1)
$$

we find the detailed (rescaled) asymptotics of $A_{n, m}, B_{n, m}$ and $P_{n, m}$ on $\mathbb{C}$, and describe the phase transitions of this limit behavior as a function of $\alpha$. This description is given in terms of the vector critical measure $\vec{\mu}_{\alpha}=\left(\mu_{1}, \mu_{2}, \mu_{3}\right)$, the saddle point of an energy functional comprising both attracting and repelling forces. This critical measure is characterized by a cubic equation (spectral curve), and its components $\mu_{j}$ live on trajectories of a canonical quadratic differential $\varpi$ on the Riemann surface of this equation. The structure of these trajectories and their deformations as functions of $\alpha$ was object of study in our previous paper [Adv. Math. 302 (2016), 1137-1232], and some of the present results strongly rely on the analysis carried out there.

We conclude that the asymptotic zero distribution of the polynomials $A_{n, m}$ and $P_{n, m}$ are given by appropriate combinations of the components $\mu_{j}$ of the vector critical measure $\vec{\mu}_{\alpha}$. However, in the case of the zeros of $B_{n, m}$ the behavior is totally different, and can be described in terms of the balayage of the signed measure $\mu_{2}-\mu_{3}$ onto certain curves on the plane. These curves are constructed with the aid of the canonical quadratic differential $\varpi$, and their topology has three very distinct characters, depending on the value of $\alpha$, and are obtained from the critical graph of $\varpi$.

Once the trajectories and vector critical measures are studied, the main asymptotic technical tool is the Deift-Zhou nonlinear steepest descent analysis of a $3 \times 3$ matrix-valued Riemann-Hilbert problem characterizing both $\left(A_{n, m}, B_{n, m}\right)$ and $P_{n, m}$, which allows us to obtain the limit behavior of both types of MOPs simultaneously.

We illustrate our findings with results of several numerical experiments, explain the computational methodology, and formulate some conjectures and empirical observations based on these experiments.

\section{Contents}

\section{Introduction}

2010 Mathematics Subject Classification. Primary: 33C45; Secondary: 30C70, 30E10, 30F30, $31 \mathrm{~A} 15$.

Key words and phrases. Logarithmic potential theory, vector energy, equilibrium on the complex plane, critical measures, $S$-property, quadratic differentials trajectories, multiple orthogonal polynomials, asymptotics, Riemann-Hilbert problems. 
2. Statement of main results

3. Spectral curve and auxiliary functions

4. The canonical quadratic differential 18

5. The Riemann-Hilbert formulation

6. Steepest Descent Analysis 228

6.1. Preliminary transformation $\quad 29$

6.2. First transformation

6.3. Opening of lenses in the precritical case 33

6.4. Opening of lenses in the supercritical case 35]

6.5. Construction of the global parametrix 38

6.6. Parametrices near branch points 40

6.7. Final transformation 42

7. Steepest descent analysis for general $\alpha$

8. Asymptotics 45

9. Numerical experiments and empirical observations 49

Acknowledgements 51

References $\quad$ 59

\section{INTRODUCTION}

The theory of Padé approximants, which provide a locally best rational approximation to a power series, was developed in the 19th-20th centuries intertwined with the theory of continued fractions, see 4 for some historical remarks. In his 1895 paper [34] Markov (or Markoff) proved that denominators of these approximants to what we now call Cauchy transform of a positive function on the real line are orthogonal with respect to this function, linking convergence analysis of such approximants to the analytic theory of orthogonal polynomials.

Since Padé approximants allow us, at least potentially, to study the global properties of an analytic function given locally by a power series, they became a very popular tool in applications, which stimulated the rapid development of their theory in the 1970s-80s. During these years the extension to the essentially non-real situation began, first in the works of Gonchar and Nuttall, and later, of Rakhmanov and Stahl. They, together with Mhaskar and Saff, unraveled also the power of logarithmic potential theory tools.

The main challenge in this extension was that the denominators of Padé approximants for holomorphic germs of algebraic (multivalued) functions satisfy nonHermitian orthogonality conditions: they do not correspond to a scalar product and the contour of integration can be freely deformed within its homotopy class. It was Stahl [45,46] who first explained how to select the right contour (and in consequence, to establish weak or $n$-th root asymptotics for these orthogonal polynomials), showing that these contours solve a max-min problem for logarithmic energy. He also characterized them by means of a certain symmetry property (now called $S$-property), which turned out to be a very powerful tool, linking the problem to geometric function theory. In this context, finding such contours with the $S$-property then becomes equivalent to finding quadratic differentials with given critical points and desired topology of trajectories. 
Stahl's methods were improved and enhanced in the work of Gonchar and Rakhmanov 24, allowing for varying measures (those depending on the degree of the polynomial), giving rise to the Gonchar-Rakhmanov-Stahl (or GRS) theory. This is one of a few general results in the modern analytic theory of Padé approximants and non-hermitian orthogonal polynomials. A crucial ingredient of this method is the existence of contours on $\mathbb{C}$ exhibiting a weighted $S$-property: assuming the existence of such contours, it is possible to obtain asymptotics for non-hermitian orthogonal polynomials with varying weights. Stahl was able to prove existence in the nonweighted case, but it took more than 20 years for the existence of the weighted $S$-contour to be established even for polynomial external fields [32], following some ideas of Rakhmanov 42. The proof in 32 relied on the analysis of the so-called critical measures (saddle points of the weighted logarithmic energy), introduced in [35], which naturally extend the notion of equilibrium measures. Critical measures turned out to be useful also in the study of polynomial solutions of some linear ODE 35, 36, 38.

A construction generalizing continued fractions but for several functions simultaneously was put forward by Hermite 25 as the crucial step in his proof of transcendence of the number $e$; it is known today as Hermite-Padé or simultaneous approximation to a system of functions. There are two types of Hermite-Padé approximants, and as in the case of their one-dimensional ancestors, the corresponding polynomials satisfy a set of orthogonality conditions, but now involving more than one measure (or weight function). These are the Hermite-Padé (or multiple) orthogonal polynomials (shortly MOPs), see their definitions below.

Although born from the needs of approximation theory and number theory, recently MOPs attracted increasing interest due to their appearance in the analysis of several random matrix and non-intersecting random paths models, among others,

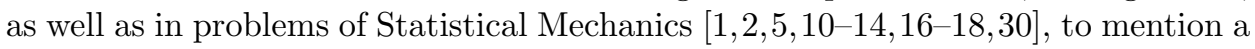
few. All these applications stimulated also the development of the analytic theory of MOPs, starting with the pioneering works of the Russian school (Aptekarev, Gonchar, Kalyagin, Nikishin, Rakhmanov, and Suetin), which were later on joined by López-Lagomasino, Nuttall, Stahl, Van Assche, among many others. In the initial stage, the study circumscribed to two somewhat extreme cases on the real line, associated with the names of Angelesco and Nikishin, when existence of all polynomials of their maximal degree is guaranteed (the so-called normality of these approximants, see [19]). Gonchar and Rakhmanov showed 22,23] that the large degree limit of the zeros of MOPs for Angelesco systems of measures is described by a vector equilibrium problem involving mutually repelling and disjointly supported measures on the real line, while for the Nikishin systems we need to consider vector equilibrium where attractive interactions are present.

However, extending this methodology to the complex case and for multivalued functions without real symmetry turned out to be a formidable task. The asymptotic analysis of the corresponding Hermite-Padé polynomials satisfying nonHermitian orthogonality conditions requires considering new non-standard vector equilibria (involving additional measures) with new types of constraints and a new notion of $S$-property. In such settings, the mere existence of solution of the corresponding vector max-min problems is not guaranteed. Hence, despite many efforts and recent contributions $3,6,9,26,27,29,33,41,43$, it looks like a multidimensional extension of the GRS theory, valid for MOPs with arbitrary number of weights and 
no underlying symmetries, is nowhere in sight. This is why the analysis of even simplest non-trivial cases that do not exhibit any particular symmetry can shed a new light onto the problem.

In an effort to extend the GRS theory to a general context of multiple nonHermitian orthogonality, we carried out in our previous work 37 a systematic study of vector critical measures, and found a characterization valid for systems comprising both "Angelesco" and "Nikishin" type interactions on the plane. We showed that for our choice of interaction matrices, the condition that a vector of measures is critical is equivalent to their Cauchy transforms being the solutions (in a sense specified in [37]) of an algebraic equation, also known as the spectral curve. Furthermore, we demonstrated that the critical measures live on trajectories of a canonical quadratic differential on the compact Riemann surface associated to the spectral curve. This way, we can embed the support of the critical measures on this Riemann surface, in such a way that different pairwise interactions, such as Angelesco and Nikishin, can be put on equal foot. For a cubic potential, we illustrated the power of the theory of quadratic differentials in this setup, by explicitly constructing a one-parametric family of vector critical measures through the precise dynamical description of the trajectories of the underlying quadratic differential.

The interaction matrix considered in 37 appears also in the analysis of random matrix models with external source. For the general quartic potential with even symmetry, and about at the same time, for the general even degree potential with even symmetry, Aptekarev, Lysov and Tulyakov [7,8] and Bleher, Delvaux and Kuijlaars [11], respectively, addressed such matrix model using the MOPs approach. The key object that allowed them to obtain several asymptotic results was a vector equilibrium problem. The vector equilibrium problems considered in 7,8 and [1] are essentially different, but in both situations their derivation relies on the strong symmetry of the problem. Under this perspective, our choice of interaction matrix in 37 generalizes the explicit construction of Aptekarev, Lysov and Tulyakov to potentials without symmetries, and (as an ongoing research shows) can be reduced to the constrained vector equilibrium problem considered by Bleher, Delvaux and Kuijlaars.

The present work is a natural continuation of [37]: we analyze a family of nonHermitian MOPs (both of types I and II) with respect to a cubic weight and find their asymptotic description in terms of the vector critical measures constructed in [37. It should be pointed out that the large degree zero distribution of these polynomials is highly non-trivial, exhibiting several phase transitions, as Figures 1 and 2 illustrate. Some previous contributions 3, 3] addressed the large degree asymptotics on the plane in the non-symmetric case and for weights on bounded sets having only finite branch points; see also [26 27] for nice numerical experiments and empirical discussion related to the so-called Nutall's conjecture. To our knowledge, the present work is the first systematic study of zero distribution of MOPs with complex zeros that exhibit non-hermitian orthogonality on unbounded sets (leading to consideration of extremal problems with an external field) with no real symmetry. As we hope it will become clear to the reader, the novelty fact that explains such break of symmetry is the canonical quadratic differential on the spectral curve and its trajectories, that were rigorously described in our previous work [37. Once these trajectories are well understood, the dynamics of the limiting zero distribution can 


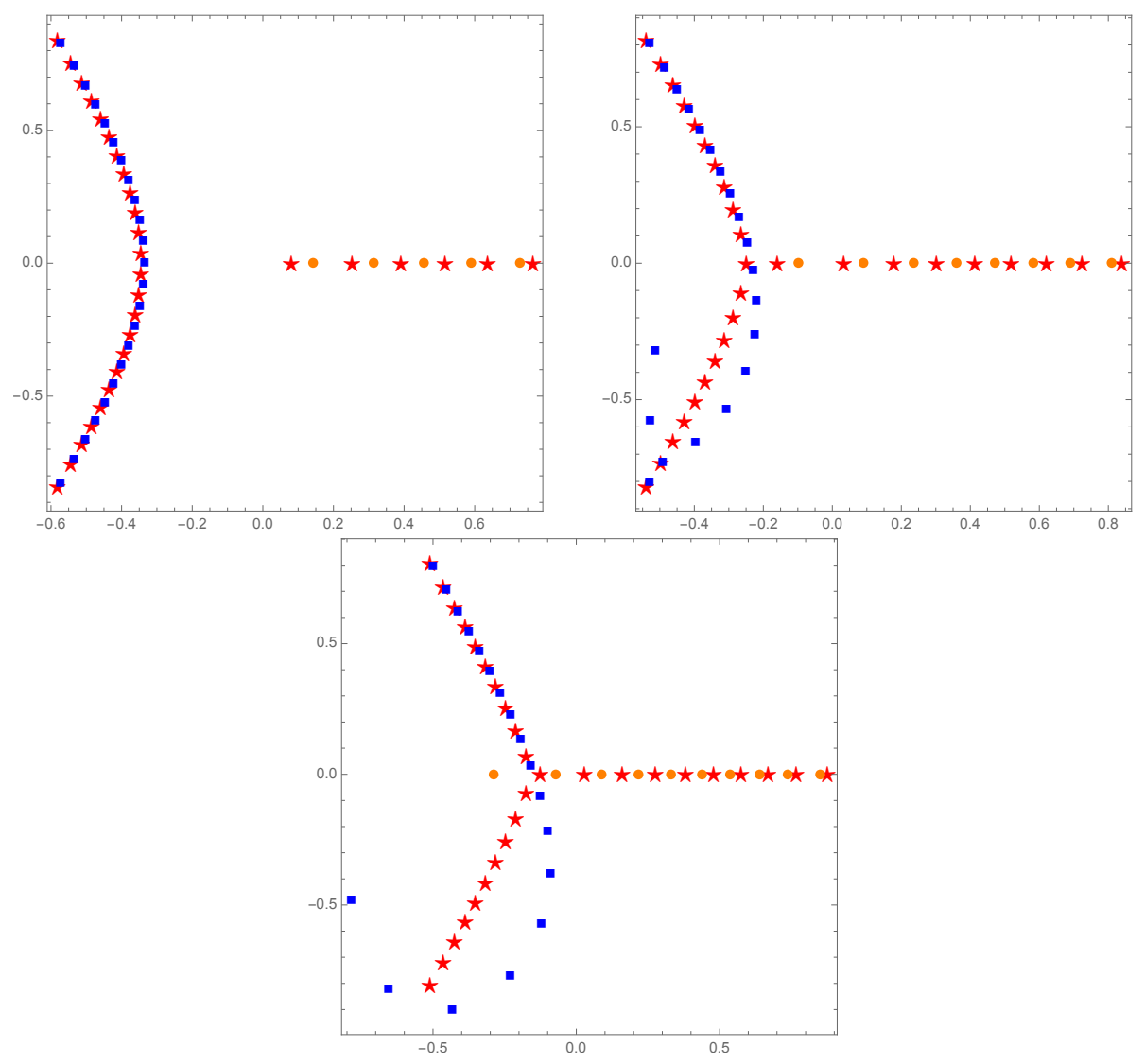

Figure 1. Zeros of $P_{n, m}$ (stars), $A_{n, m}$ (dots) and $B_{n, m}$ (squares), for $n+m=30$ fixed and the choices $(n, m)=(6,24)$ (top left), $(n, m)=(9,21)$ (top right) and $(n, m)=(11,19)$ (bottom); see Definitions 2.1 and 2.2 .

be read off from these trajectories, and the very distinct behaviors portrayed in Figures Figures 1 and 2 are easily understood under the very same framework.

Besides the aforementioned quadratic differentials that were previously studied in 37, our main asymptotic tool is the matrix Riemann-Hilbert problem characterization of MOPs 21] with cubic weights and the development of the Deift-Zhou nonlinear steepest descent method that yields not only the limiting zero distribution but a detailed uniform asymptotics of the polynomial on the whole complex plane. In order to be able to perform the necessary steps of this approach we had to introduce a preliminary transformation that although looked purely technical in the first stage, turned out to be crucially related to the core of the matter. Moreover, the quadratic differential studied in 37. played a fundamental role in the construction of the leading term of the asymptotics, and its critical graph (with all its phase transitions) was an essential guide during the deformations of contours required to complete the steepest descent analysis.

The structure of the paper is as follows. In the next section we state our main findings that explain in particular the numerical outcomes illustrated in Figures 1 


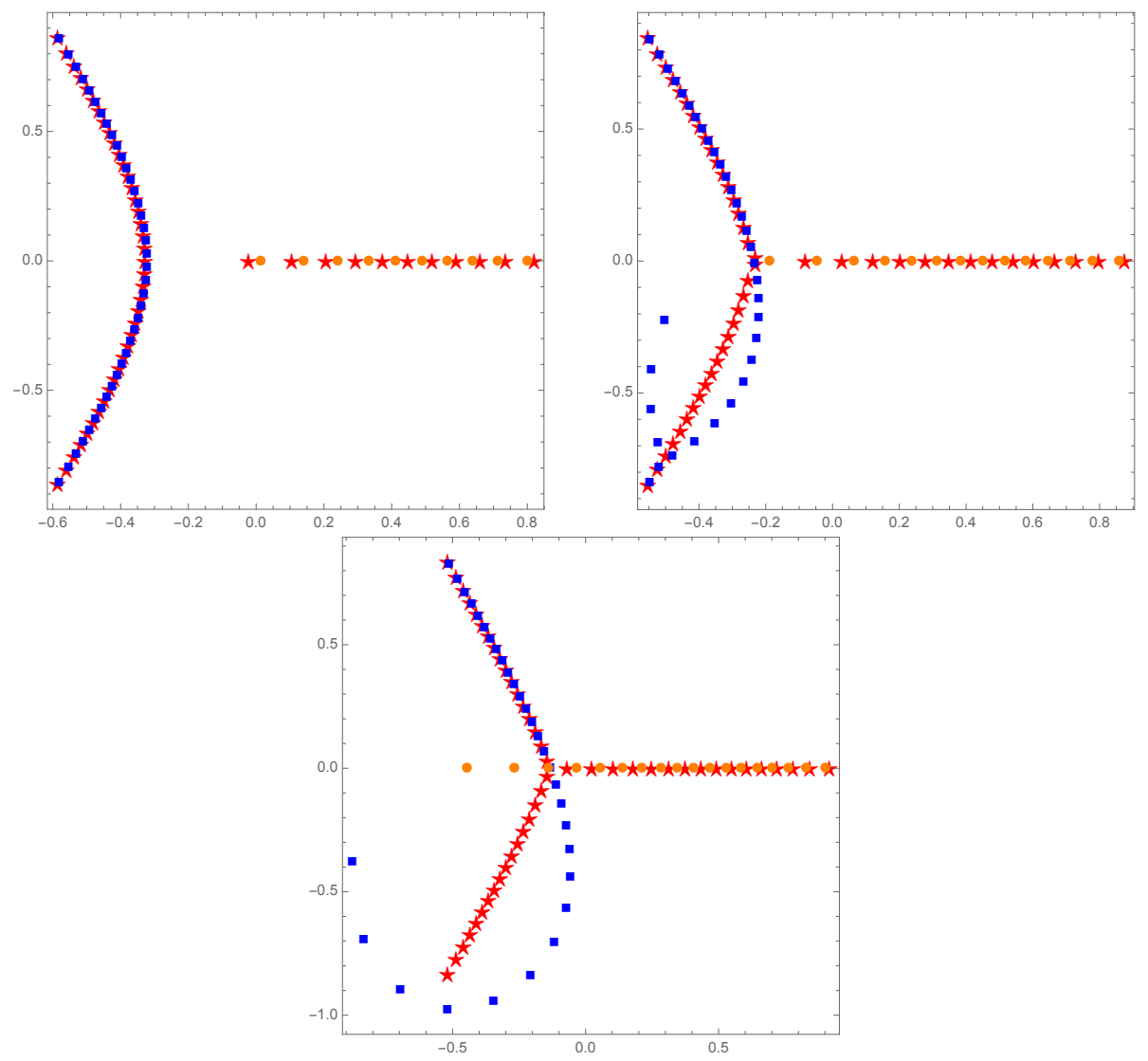

Figure 2. Zeros of $P_{n, m}$ (stars), $A_{n, m}$ (dots) and $B_{n, m}$ (squares), for $n+m=50$ fixed and the choices $(n, m)=(11,39)$ (top left), $(n, m)=(15,35)$ (top right) and $(n, m)=(19,31)$ (bottom); see Definitions 2.1 and 2.2 .

and 2. for which we need to provide a minimum background from 37. However, the proofs require the use of several technical results from 37] that for convenience of the reader we gather in Sections 3 and 4 . As it was mentioned, our approach to asymptotics is based on the Riemann-Hilbert characterization of the MOPs (Section 5. and on the corresponding Deift-Zhou steepest descent analysis. It consists of several transformations, described in Section 6. We can use the result of this analysis to derive the strong (locally uniform) asymptotics of both type I and type II MOPs, and as a consequence, describe their limit zero behavior (Section 8), proving in such a way our main assertions made in Section 2 Finally, in Section 9 we explain the computational methodology used for our numerical experiments and formulate some conjectures and empirical observations.

\section{Statement of main Results}

Consider two contours $\gamma_{1}$ and $\gamma_{2}$ on the complex plane, extending to $\infty$ on their two ends along the directions determined by the angles $-\pi / 3$ and 0 , and $-\pi / 3$ and 
$\pi / 3$, respectively (see Figure 12 , left). Then expressions

$$
\begin{aligned}
& \mathfrak{f}_{1}(z)=\int_{\gamma_{1}} \frac{e^{-t^{3}}}{z-t} d t, \quad z \in \mathbb{C} \backslash \gamma_{1}, \\
& \mathfrak{f}_{2}(z)=\int_{\gamma_{2}} \frac{e^{-t^{3}}}{z-t} d t, \quad z \in \mathbb{C} \backslash \gamma_{2},
\end{aligned}
$$

define holomorphic functions in the respective domains. Their corresponding asymptotic expansions at infinity are the formal power series (that we again denote by $\left.\mathfrak{f}_{i}\right)$,

with

$$
\mathfrak{f}_{i}(z)=\sum_{k=0}^{\infty} \frac{\mathfrak{f}_{i}^{(k)}}{z^{k+1}}, \quad \mathfrak{f}_{i}^{(k)} \stackrel{\text { def }}{=} \int_{\gamma_{i}} t^{k} e^{-t^{3}} d t, \quad i=1,2 .
$$

$$
\begin{aligned}
\mathfrak{f}_{1}^{(k)} \stackrel{\text { def }}{=} \int_{\gamma_{1}} t^{k} e^{-t^{3}} d t=\frac{1}{3} \Gamma\left(\frac{k+1}{3}\right)\left(e^{2 \pi i(k+1) / 3}-1\right), \\
\mathfrak{f}_{2}^{(k)} \stackrel{\text { def }}{=} \int_{\gamma_{2}} t^{k} e^{-t^{3}} d t=\frac{2 i}{3} \Gamma\left(\frac{k+1}{3}\right) \sin (2 \pi i(k+1) / 3) .
\end{aligned}
$$

Given integer values $n, m \geq 0$, denote $N \stackrel{\text { def }}{=} n+m$. Type I Hermite-Padé (or simultaneous) approximants to the pair of functions $\left(\mathfrak{f}_{1}, \mathfrak{f}_{2}\right)$ are constructed by finding polynomials $a_{n, m}, b_{n, m}$, with $\operatorname{deg} a_{n, m} \leq n-1$, $\operatorname{deg} b_{n, m} \leq m-1$, not simultaneously identically zero, such that for some polynomial $d_{n, m}$,

$$
a_{n, m}(z) \mathfrak{f}_{1}(z)+b_{n, m}(z) \mathfrak{f}_{2}(z)-d_{n, m}(z)=\mathcal{O}\left(\frac{1}{z^{N}}\right), \quad z \rightarrow \infty
$$

Type II Hermite-Padé (or simultaneous) approximants to $\left(\mathfrak{f}_{1}, \mathfrak{f}_{2}\right)$ is the pair of rational functions $\left(q_{n, m}^{(1)} / p_{n, m}, q_{n, m}^{(2)} / p_{n, m}\right)$, where $p_{n, m} \not \equiv 0$ is of degree $\leq N$, and $q_{n, m}^{(j)}$ are polynomials such that

$$
\begin{aligned}
& p_{n, m}(z) \mathfrak{f}_{1}(z)-q_{n, m}^{(1)}(z)=\mathcal{O}\left(\frac{1}{z^{n}}\right), \quad z \rightarrow \infty, \\
& p_{n, m}(z) \mathfrak{f}_{2}(z)-q_{n, m}^{(2)}(z)=\mathcal{O}\left(\frac{1}{z^{m}}\right), \quad z \rightarrow \infty,
\end{aligned}
$$

see e.g. [40, Chapter 4]. Standard arguments using Cauchy formula allow to show that $a_{n, m}, b_{n, m}$ and $p_{n, m}$ satisfy non-hermitian orthogonality conditions that we formulate next, after appropriate rescaling.

Definition 2.1. Given $n, m \in \mathbb{N}$ and $N=n+m$, the type I multiple orthogonal polynomials $A_{n, m}$ and $B_{n, m}$, if they exist, are uniquely defined by the following conditions:

$$
\operatorname{deg} A_{n, m} \leq n-1, \quad \operatorname{deg} B_{n, m} \leq m-1,
$$

and

$$
\begin{aligned}
& \int_{\gamma_{1}} z^{k} A_{n, m}(z) e^{-N z^{3}} d z+\int_{\gamma_{2}} z^{k} B_{n, m}(z) e^{-N z^{3}} d z=0, \quad k=0, \ldots, N-2, \\
& \int_{\gamma_{1}} z^{k} A_{n, m}(z) e^{-N z^{3}} d z+\int_{\gamma_{2}} z^{k} B_{n, m}(z) e^{-N z^{3}} d z=1, \quad k=N-1 .
\end{aligned}
$$

The dual notion is 
Definition 2.2. Given $n, m \in \mathbb{N}$ and $N=n+m$, we define the type II multiple orthogonal polynomial $P_{n, m}$, if it exists, to be the unique monic polynomial of degree $N\left(\operatorname{deg} P_{n, m}=N\right)$ that fulfills the conditions

$$
\begin{aligned}
& \int_{\gamma_{1}} z^{k} P_{n, m}(z) e^{-N z^{3}} d z=0, \quad k=0, \ldots, n-1, \\
& \int_{\gamma_{2}} z^{k} P_{n, m}(z) e^{-N z^{3}} d z=0, \quad k=0, \ldots, m-1 .
\end{aligned}
$$

Both (2.2) and (2.3) give rise to a non-homogeneous linear system of $n+m=N$ equations on the $N$ (unknown) coefficients of $P_{n, m}$ (in the case of $(2.3)$ ) or of the two polynomials $A_{n, m}, B_{n, m}$ otherwise. Hence if the solution exists, it is unique. However, in contrast to the standard orthogonality on the real line, the existence of $P_{n, m}$ or of $A_{n, m}, B_{n, m}$ is a non-trivial matter (and actually not true for general systems of weights).

The main object of our study is the asymptotic analysis of the type I and type II polynomials defined above, in the limit when $N=n+m \rightarrow \infty$ in such a way that

$$
\frac{n}{N} \longrightarrow \alpha \in[0,1]
$$

It should be noted that for (2.3) the extremal cases $n=0$ and $n=m$ (for type II polynomials) have been studied previously by Deaño, Huybrechs and Kuijlaars 15 and Filipuk, Van Assche and Zhang [20], respectively; in fact, these studies were the original motivation for [37] and the present work.

We can restrict our analysis to the case

$$
\frac{n}{N} \longrightarrow \alpha \in(0,1 / 2)
$$

since we can easily derive conclusions for $\alpha \in(1 / 2,1)$ by mapping $\alpha \mapsto 1-\alpha$, swapping the role of $\mathfrak{f}_{1}$ and $\mathfrak{f}_{2}$ and rotating the complex plane by $2 \pi / 3$.

As it was mentioned, we make use of the Riemann-Hilbert formulation of the multiple orthogonality that characterizes both $P_{n, m}$ and $A_{n, m}, B_{n, m}$, see [21] and Section 5 below. The advantage of this approach is that it gives the uniform asymptotics of these polynomials on every subdomain of the complex plane and that it allows to perform the asymptotic analysis valid simultaneously for both families of polynomials. The limiting behavior is described in terms of the solution of a vector equilibrium problem and of some abelian integrals on the associated Riemann surface, whose construction relies on the analysis carried out in 37.

For a compactly supported Borel measure $\mu$, its logarithmic potential and Cauchy transform are defined by

$$
U^{\mu}(z)=\int \log \frac{1}{|s-z|} d \mu(s), \quad C^{\mu}(z)=\lim _{\varepsilon \rightarrow 0} \int_{|s-z|>\varepsilon} \frac{d \mu(s)}{s-z}, \quad z \in \mathbb{C},
$$

respectively. $U^{\mu}$ is subharmonic on $\mathbb{C}$ and harmonic on $\mathbb{C} \backslash \operatorname{supp} \mu$, whereas $C^{\mu}$ is well defined and finite a.e. with respect to planar Lebesgue measure, and it is analytic on $\mathbb{C} \backslash \operatorname{supp} \mu$. For

$$
\partial_{z}=\frac{1}{2}\left(\frac{\partial}{\partial x}-i \frac{\partial}{\partial y}\right)
$$

these functions are related through the equality

$$
2 \partial_{z} U^{\mu}=C^{\mu}
$$


which should be understood in the strong sense for $z \in \mathbb{C} \backslash \operatorname{supp} \mu$ and in the distributional sense on $\operatorname{supp} \mu$.

For a given vector of three non-negative measures $\vec{\mu}=\left(\mu_{1}, \mu_{2}, \mu_{3}\right)$, the nonnegative definite interaction matrix

$$
A=\left(a_{j k}\right)=\left(\begin{array}{ccc}
1 & \frac{1}{2} & \frac{1}{2} \\
\frac{1}{2} & 1 & -\frac{1}{2} \\
\frac{1}{2} & -\frac{1}{2} & 1
\end{array}\right)
$$

and the external field $\phi(z)=\operatorname{Re}\left(z^{3}\right)$, we consider the energy functional

$$
E(\vec{\mu})=\sum_{j, k=1}^{3} a_{j k} I\left(\mu_{j}, \mu_{k}\right)+\int \phi(z) d \mu_{1}(z)+\int \phi(z) d \mu_{2}(z),
$$

where

$$
I(\mu, \nu)=\iint \log \frac{1}{|x-y|} d \mu(x) d \nu(y)
$$

is the logarithmic interaction between the two measures $\mu, \nu$.

For a fixed number $\alpha \in[0,1 / 2)$, we restrict the energy $E(\cdot)$ on the class $\mathcal{M}_{\alpha}$ of vectors of measures $\vec{\mu}$ satisfying the following assumptions.

- The components $\mu_{1}, \mu_{2}$ and $\mu_{3}$ of $\vec{\mu} \in \mathcal{M}_{\alpha}$ are compactly supported nonnegative Borel measures on $\mathbb{C}$, their supports have zero planar Lebesgue measure and furthermore the energy $E(\vec{\mu})$ is finite.

- The total masses of $\mu_{1}, \mu_{2}$ and $\mu_{3}$ are related through

$$
\left|\mu_{1}\right|+\left|\mu_{2}\right|=1, \quad\left|\mu_{1}\right|+\left|\mu_{3}\right|=\alpha, \quad\left|\mu_{2}\right|-\left|\mu_{3}\right|=1-\alpha .
$$

- The set

is finite.

$$
\bigcup_{1 \leq j<k \leq 3}\left(\operatorname{supp} \mu_{j} \cap \operatorname{supp} \mu_{k}\right)
$$

In 37. we extended the notion of critical measures introduced by the first author and Rakhmanov to the vector energy setting. In the present context, this extension reads as follows. For $t \in \mathbb{C}$ and $h \in C^{2}(\mathbb{C})$, denote by $\mu^{t}$ the pushforward of the scalar measure $\mu$ induced by the transformation $z \mapsto z+\operatorname{th}(z), z \in \mathbb{C}$. For $\mu \in \mathcal{M}_{\alpha}$, we denote $\vec{\mu}^{t}=\left(\mu_{1}^{t}, \mu_{2}^{t}, \mu_{3}^{t}\right)$ and say that $\vec{\mu}$ is critical if

$$
\lim _{t \rightarrow 0} \frac{E\left(\vec{\mu}^{t}\right)-E(\mu)}{t}=0,
$$

for every $h \in C^{2}(\mathbb{C})$.

A summary of the results proven in 37] that we need here is given by the next theorem.

Theorem 2.3 ( 37 , Theorems 1.12 and 1.14]). For each $\alpha \in(0,1 / 2)$ there exists a vector critical measure $\vec{\mu}_{\alpha} \in \mathcal{M}_{\alpha}$ for the energy $E(\cdot)$ defined in 2.4. Furthermore, the combinations of Cauchy transforms

$$
\begin{aligned}
& \xi_{1}(z)=2 z^{2}+C^{\mu_{1}}(z)+C^{\mu_{2}}(z), \\
& \xi_{2}(z)=-z^{2}-C^{\mu_{1}}(z)-C^{\mu_{3}}(z), \\
& \xi_{3}(z)=-z^{2}-C^{\mu_{2}}(z)+C^{\mu_{3}}(z)
\end{aligned}
$$

satisfy a.e. the algebraic equation (also called spectral curve)

$$
\xi^{3}-R(z) \xi+D(z)=0
$$



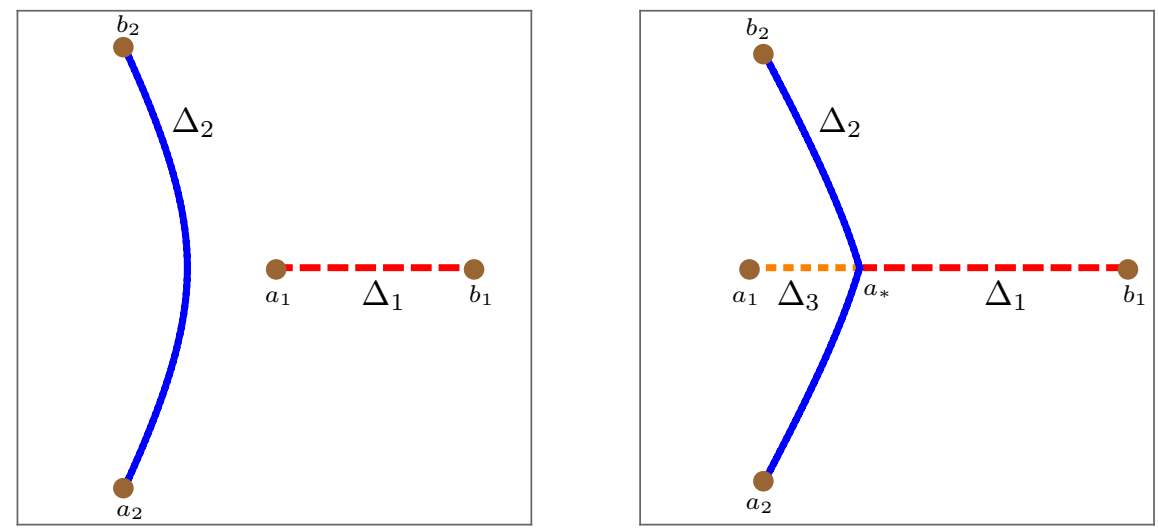

Figure 3. (Extracted from 37) For $\alpha \approx 0.14786<\alpha_{c} \approx$ 0.2578357 (left panel) and for $\alpha \approx 0.348342>\alpha_{c}$ (right panel), the output of the numerical evaluation of $\Delta_{1} \stackrel{\text { def }}{=} \operatorname{supp} \mu_{1}$ (long dashed line), $\Delta_{2} \stackrel{\text { def }}{=} \operatorname{supp} \mu_{2}$ (continuous line) and $\Delta_{3} \stackrel{\text { def }}{=} \operatorname{supp} \mu_{3}$ (short dashed line - only on the right panel).

where

$$
R(z)=3 z^{4}-3 z-c, \quad D(z)=-2 z^{6}+3 z^{3}+c z^{2}-3 \alpha(1-\alpha),
$$

with $c=c(\alpha)$ the real parameter given by

$$
c=-\left(\frac{243}{64}(1-4 \alpha(1-\alpha))^{2}\right)^{\frac{1}{3}} .
$$

The support of each of the measures $\mu_{1}$ and $\mu_{3}$ is a single interval of the real line, whereas supp $\mu_{2}$ is a simple piece-wise analytic arc, symmetric with respect to complex conjugation, that connects two complex conjugate points.

Finally, there exists a critical value $\alpha_{c} \approx 0.2578357$ for which

(i) (subcritical regime) if $\alpha<\alpha_{c}$, then $\operatorname{supp} \mu_{3}=\emptyset$ and $\mu_{1}$ and $\mu_{2}$ are disjointly supported non-trivial measures;

(ii) (supercritical regime) if $\alpha>\alpha_{c}$, then the three measures $\mu_{1}, \mu_{2}$ and $\mu_{3}$ are non-trivial and their supports have one common intersection point, $a_{*}$.

The measures $\mu_{1}, \mu_{2}$ and $\mu_{3}$ obviously depend on $\alpha$, so whenever necessary we write $\mu_{j}=\mu_{\alpha, j}$ to emphasize this dependency.

We denote the endpoints of $\operatorname{supp} \mu_{2}$ by $a_{2}$ and $b_{2}$, with the convention that $\operatorname{Im} a_{2}<0$. Furthermore, in the subcritical case, $\operatorname{supp} \mu_{1}=\left[a_{1}, b_{1}\right]$ and in the supercritical case, $\operatorname{supp} \mu_{1}=\left[a_{*}, b_{1}\right]$ and $\operatorname{supp} \mu_{3}=\left[a_{1}, a_{*}\right]$. In either cases, the points $a_{1}, a_{2}, b_{1}$ and $b_{2}$ are branch points of the spectral curve (2.7), and $a_{*}$ is also the unique intersection point of $\operatorname{supp} \mu_{2}$ with $\mathbb{R}$.

The structure of $\Delta_{j}=\operatorname{supp} \mu_{j}, j=1,2,3$ (based on actual numerical evaluation), is displayed in Figure 3 ,

The existence of the vector critical measure $\vec{\mu}$ claimed by Theorem 2.3 is highly non-trivial, and a great portion of the work 37 is dedicated to this proof. In 37] the measures $\mu_{1}, \mu_{2}$ and $\mu_{3}$ are described rather explicitly in terms of critical trajectories of a quadratic differential defined on the Riemann surface associated to 

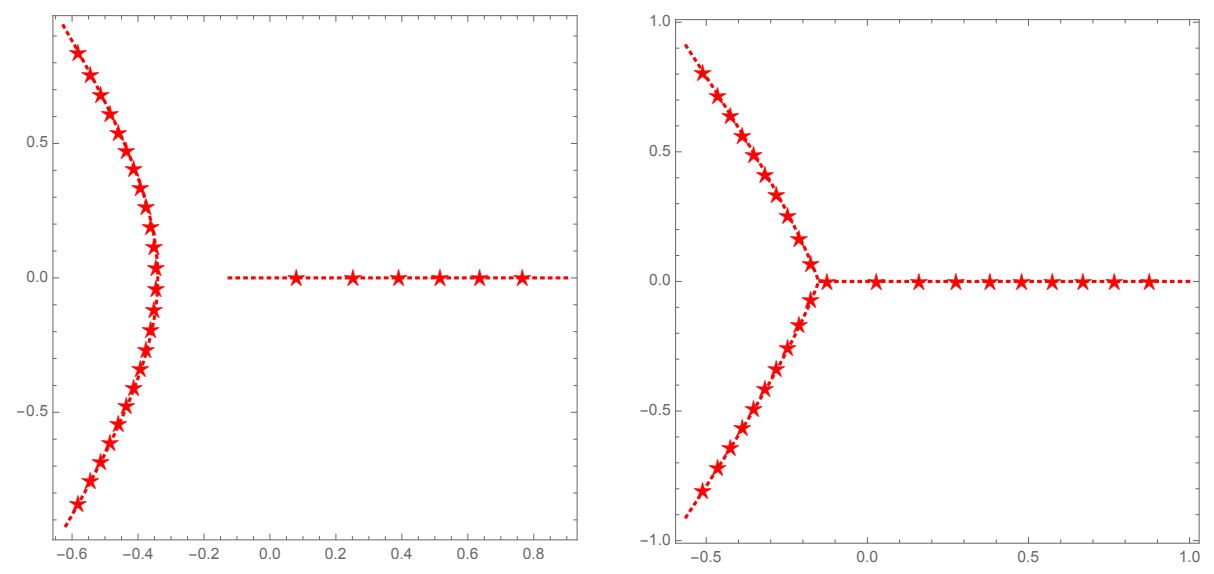

Figure 4. Zeros of $P_{6,24}\left(\alpha=0.2<\alpha_{c}\right.$, left $)$ and $P_{11,19}(\alpha \approx$ $0.366>\alpha_{c}$, right), along with the support of $\mu_{1}+\mu_{2}$.

the spectral curve 2.7). This quadratic differential also plays a fundamental role in the analysis of the $g$-functions carried out in Section 6 below.

Denote by

$$
\nu(Q) \stackrel{\text { def }}{=} \frac{1}{N} \sum_{Q(w)=0} \delta_{w},
$$

where $Q$ is one of the polynomials $P_{n, m}, A_{n, m}$ and $B_{n, m}$, if it exist. Notice that each zero is counted in the sum above according to its multiplicity.

As a first result, we relate the weak limit, which we denote by $\stackrel{*}{\rightarrow}$, of the zero counting measures for the type I $\left(A_{n, m}\right)$ and type II $\left(P_{n, m}\right)$ polynomials with the vector critical measure given by Theorem 2.3

Theorem 2.4. Let $\alpha \in(0,1 / 2) \backslash\left\{\alpha_{c}\right\}$. If $\vec{\mu}_{\alpha}=\left(\mu_{1}, \mu_{2}, \mu_{3}\right)$ is the corresponding vector critical measure given by Theorem 2.3. then for all sufficiently large $n$ and $m$ such that

$$
\frac{n}{N} \longrightarrow \alpha
$$

polynomials $P_{n, m}$ and $A_{n, m}$ exist, and

$$
\nu\left(P_{n, m}\right) \stackrel{*}{\rightarrow} \mu_{1}+\mu_{2}, \quad \nu\left(A_{n, m}\right) \stackrel{*}{\rightarrow} \mu_{1}+\mu_{3} .
$$

For illustration, the support of $\mu_{1}+\mu_{2}$ together with the zeros of $P_{n, m}$ for various choices of $n, m$ are shown in Figure 4 , whereas the zeros of $A_{n, m}$ are shown in Figures 5 and 6 .

Now we switch to the description of the weak limit of the zero-counting measure for polynomials $B_{n, m}$.

Recall [44, Ch. II] that if $\Gamma$ is a closed set of positive capacity on $\mathbb{C}$ and $\mu$ is a positive measure on $\mathbb{C}$, the balayage of $\mu$ onto $\Gamma$ gives us a unique measure, denoted by $\nu=\operatorname{Bal}(\mu ; \Gamma)$, such that $\operatorname{supp} \nu \subset \Gamma$,

$$
|\nu|=|\mu|, \quad U^{\nu}(z)=U^{\mu}(z)+\kappa, \quad \text { quasi-everywhere on } \Gamma,
$$

for some constant $\kappa$. For a signed measure $\mu=\mu_{+}-\mu_{-}$we define its balayage by $\operatorname{Bal}(\mu ; \Gamma) \stackrel{\text { def }}{=} \operatorname{Bal}\left(\mu_{+} ; \Gamma\right)-\operatorname{Bal}\left(\mu_{-} ; \Gamma\right)$. 
The following theorem shows that for $\alpha \in(0,1 / 2)$, among all connected sets containing $b_{2}$ and at least one of the two points $a_{1}$ and $a_{2}$ there is a unique set $E_{\alpha}$, made of analytic arcs, such that $\operatorname{Bal}\left(\mu_{2}-\mu_{3} ; E_{\alpha}\right)$ is a positive measure, which will be precisely the asymptotic zero distribution of $B_{n, m}$ when $n / N \rightarrow \alpha$.

We will denote

$$
\mathbb{H}_{+} \stackrel{\text { def }}{=}\{z \in \mathbb{C} \mid \operatorname{Im} z>0\}, \quad \mathbb{H}_{-} \stackrel{\text { def }}{=}\{z \in \mathbb{C} \mid \operatorname{Im} z<0\} .
$$

Theorem 2.5. Let $\alpha \in(0,1 / 2) \backslash\left\{\alpha_{c}\right\}$. If $\vec{\mu}_{\alpha}=\left(\mu_{1}, \mu_{2}, \mu_{3}\right)$ is the corresponding vector critical measure given by Theorem 2.3, then for all sufficiently large $n$ and $m$ such that

$$
\frac{n}{N} \longrightarrow \alpha
$$

the polynomials $B_{n, m}$ exist and there exists the weak limit

$$
\nu\left(B_{n, m}\right) \stackrel{*}{\rightarrow} \mu_{B}
$$

The measure $\mu_{B}$ is the balayage of $\mu_{2}-\mu_{3}$ onto a set $E_{\alpha}$, a finite union of analytic arcs with the following properties:

(i) (subcritical regime) if $0<\alpha<\alpha_{c} \approx 0.2578357$, then $E_{\alpha}=\Delta_{2}=\operatorname{supp} \mu_{2}$ and $\mu_{B}=\mu_{2}$.

(ii) (intermediate regime) if $\alpha_{c}<\alpha<\alpha_{2} \approx 0.354933$, then $E_{\alpha}$ is the union of three analytic arcs starting from $a_{1}, a_{2}$ and $b_{2}$, and ending at one common point $a_{B} \in \Delta_{2}$ on the lower half plane $\mathbb{H}_{-}$. Furthermore,

$$
E_{\alpha} \cap \mathbb{H}_{+}=\Delta_{2} \cap \mathbb{H}_{+} \text {. }
$$

(iii) (supercritical regime) for $\alpha>\alpha_{2}$, the set $E_{\alpha}$ is a single analytic arc from $b_{2}$ to $a_{1}$, and 2.11) still holds.

For illustration, the support of $\mu_{B}$ together with the zeros of $B_{n, m}$ for various choices of $\alpha$ are shown in Figures 5 and 6 .

Remark 2.6. The restrictions $\alpha \notin\{0,1 / 2\}$ in Theorems 2.4 2.4 are made only to be consistent with our previous work 37. Nevertheless, the case $n=0$ (corresponding to $\alpha=0$ ) was previously studied by Deaño, Huybrechs and Kuijlaars [15]. In this case, $P_{0, m}=B_{0, m}, A_{0, m}=0, \mu_{1}=\mu_{3}=0$ and $\left|\mu_{2}\right|=1$. Filipuk, Van Assche and Zhang [20] studied the polynomial $P_{n, m}$ for arbitrary, but finite, $n$ and $m$. When $n=m$ (which corresponds to $\alpha=1 / 2$ ), the polynomial $P_{n, n}$ is invariant under the rotation $z \mapsto e^{2 \pi i / 3} z$, and using this symmetry they could also obtain asymptotics of $P_{n, n}$ as $n \rightarrow \infty$. In our language, their results say that

$$
\left.d \mu_{2}(z)\right|_{\arg z= \pm \frac{2 \pi}{3}}=d \mu_{1}\left(e^{\mp 2 \pi i / 3} z\right)
$$

and the convergence $\nu\left(P_{n, n}\right) \stackrel{*}{\rightarrow} \mu_{1}+\mu_{2}$ still takes place. However, in this situation $\operatorname{supp} \mu_{3}=(-\infty, 0]$, so the vector $\left(\mu_{1}, \mu_{2}, \mu_{3}\right)$ is not critical in the strict sense of the definition used for Theorem 2.3, where unbounded components of the support were not allowed. Polynomials of type I $A_{n, n}$ and $B_{n, n}$ were not considered in [20]; however, their limiting zero distribution still exist and satisfy Theorems 2.4 and 2.5 , with the feature of the symmetry 2.12 and that

and

$$
\left.\mu_{B}\right|_{\mathbb{H}_{+}}=\left.\mu_{2}\right|_{\mathbb{H}_{+}}
$$

$$
\operatorname{supp} \mu_{B} \cap \mathbb{H}_{-}=\left[0, e^{-\pi i / 3} \infty\right),\left.\quad d \mu_{B}(z)\right|_{\mathbb{H}_{-}}=d \mu_{3}\left(e^{-2 \pi i / 3} z\right)
$$




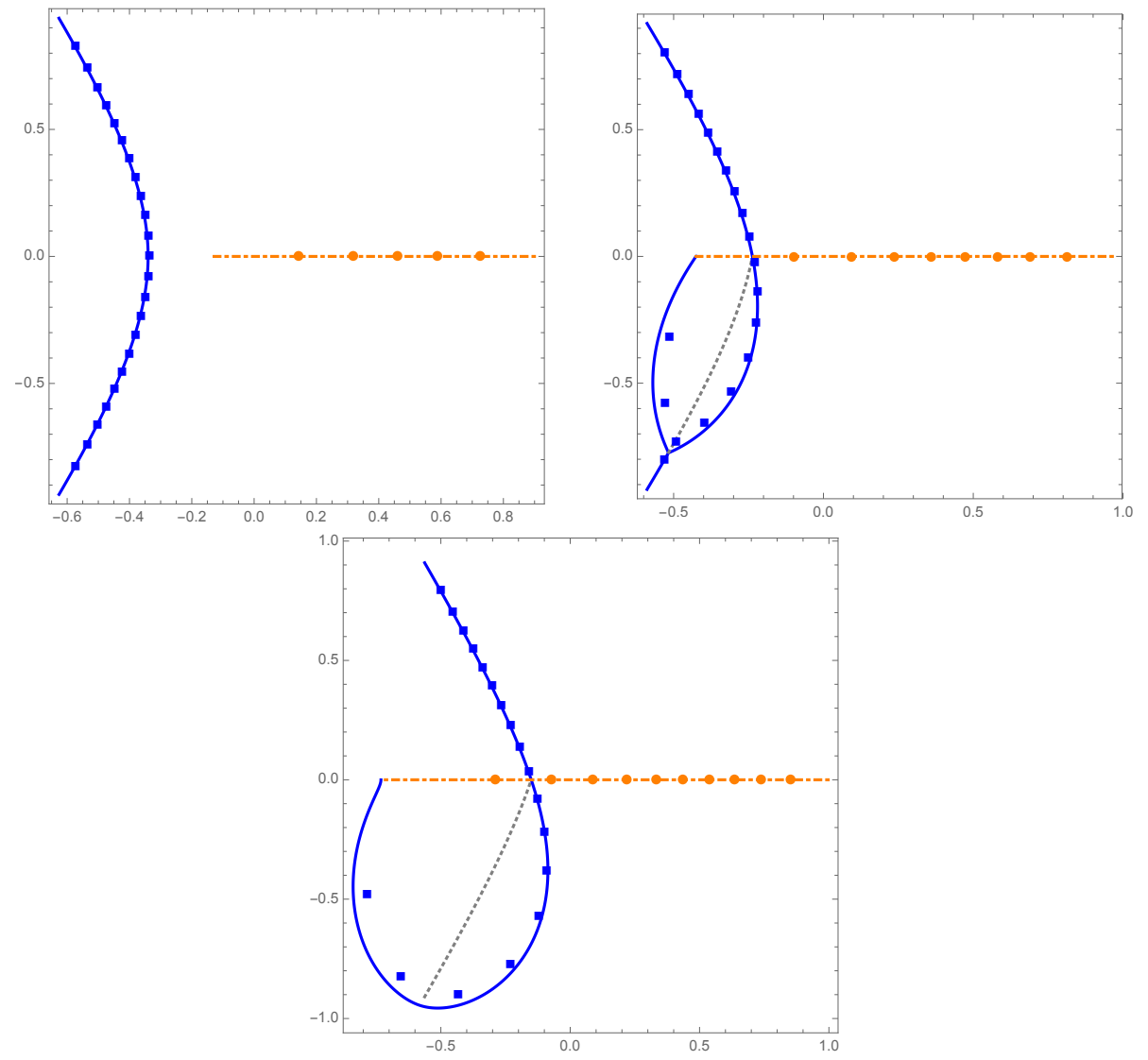

FiguRe 5 . For $n+m=30$, the zeros of $A_{n, m}$ (dots) and $B_{n, m}$ (squares), along with the supports of $\mu_{1}+\mu_{3}$ (dot-dashed lines) and $\mu_{B}$ (solid lines). They are numerical outcomes for the values $(n, m)=(6,24)\left(\alpha=0.2<\alpha_{c}\right.$, top left $),(n, m)=(9,21)(\alpha=$ $0.3 \in\left(\alpha_{c}, \alpha_{2}\right)$, top right $)$ and $(n, m)=(11,19)\left(\alpha \approx 0.366>\alpha_{2}\right.$, bottom). In the latter two, the dashed curve on the lower half plane is the part of $\operatorname{supp} \mu_{2}$ that does not coincide with $\operatorname{supp} \mu_{B}$.

We also strongly believe that Theorems 2.4 and 2.5 still hold true for $\alpha=\alpha_{c}$. For a rigorous proof in the exceptional cases just described we need to modify the Riemann-Hilbert analysis that we carry out below. Such modifications have no additional difficulty, but are lengthy, which motivated our decision to omit them for the sake of brevity.

We need to introduce further notation in order to formulate the strong asymptotic results, from which Theorems 2.4 and 2.5 readily follow.

For $\xi_{j}$ as in (2.6), we denote by $g_{j}$ their primitive functions,

$$
g_{j}(z)=\int^{z} \xi_{j}(s) d s, \quad j=1,2,3 .
$$



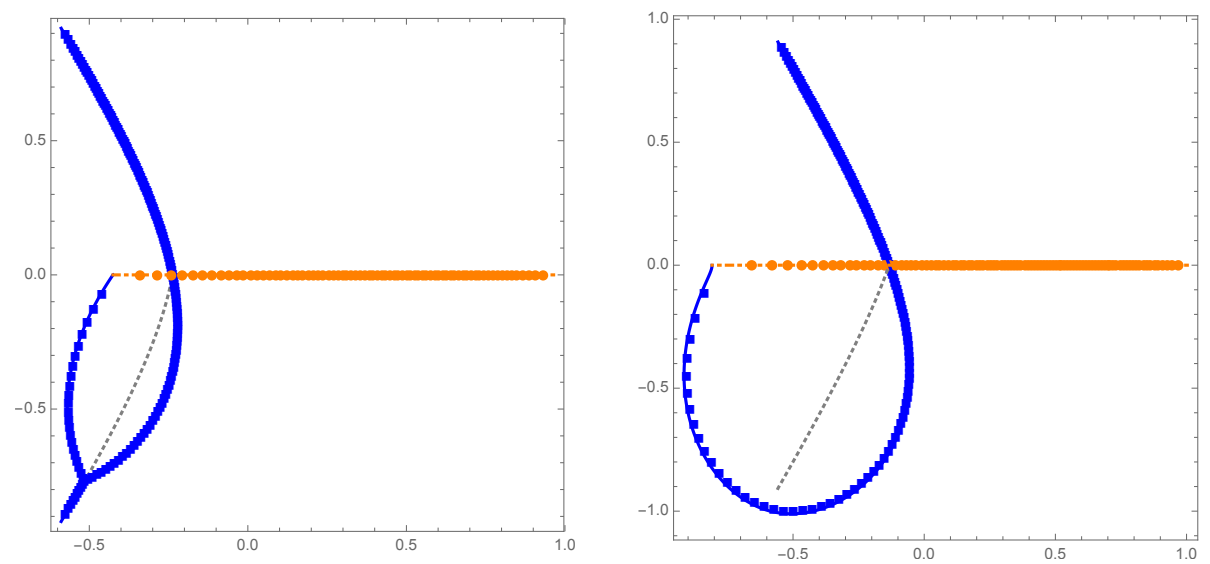

Figure 6 . For $n+m=250$, the zeros of $A_{n, m}$ (dots) and $B_{n, m}$ (squares), along with the supports of $\mu_{1}+\mu_{3}$ (dot-dashed lines), $\mu_{B}$ (solid lines) and the part of supp $\mu_{2}$ on the lower half plane that does not coincide with $\operatorname{supp} \mu_{B}$. These figures are numerical outcomes for the values $(n, m)=(75,175)\left(\alpha=0.3 \in\left(\alpha_{c}, \alpha_{2}\right)\right.$, left $)$ and $(n, m)=(95,155)\left(\alpha=0.38>\alpha_{2}\right.$, right $)$.

They have different domains of definition, $g_{1}$ is defined in $\mathbb{C} \backslash\left(\operatorname{supp} \mu_{1} \cup \operatorname{supp} \mu_{2}\right)$, $g_{2}$ in $\mathbb{C} \backslash\left(\operatorname{supp} \mu_{1} \cup \operatorname{supp} \mu_{3}\right)$, and $g_{3}$, in $\mathbb{C} \backslash\left(\operatorname{supp} \mu_{2} \cup \operatorname{supp} \mu_{3}\right)$. Furthermore, $g_{1}$ and $g_{2}$ are well defined modulo $2 \pi i$, so that functions $\exp \left(g_{1}\right)$ and $\exp \left(g_{2}\right)$ are single-valued in their domain of definition. These $g$-functions do depend on $\alpha$, so when necessary we will emphasize it by $g_{j}=g_{\alpha, j}$.

Moreover, let $f_{1}=\left(f_{11}, f_{12}, f_{13}\right)$ be a vector of holomorphic non-vanishing functions, where $f_{1 j}$ has the same domain of definition than $g_{j}, j=1,2,3$. These functions will be uniquely characterized by a Riemann-Hilbert (boundary value) problem and constructed explicitly in terms of certain abelian integral on the Riemann surface of the spectral curve 2.8 , see Section 6.5 for details. Furthermore,

$$
f_{11}(z)=1+\mathcal{O}(1 / z), \quad f_{12}(z)=\mathcal{O}(1 / z), \quad f_{13}(z)=\mathcal{O}(1 / z), \quad z \rightarrow \infty .
$$

Theorem 2.7. For $n, m$ large enough such that $\alpha_{N} \stackrel{\text { def }}{=} n / N \rightarrow \alpha \in(0,1 / 2) \backslash\left\{\alpha_{c}\right\}$, $N=n+m$, the polynomial $P_{n, m}$ exists, and for an appropriate constant $r_{1}=r_{\alpha_{N}, 1}$,

$$
P_{n, m}(z)=f_{11}(z) e^{-N\left(g_{\alpha_{N}, 1}(z)-\frac{2 z^{3}}{3}-r_{1}\right)}\left(1+\mathcal{O}\left(N^{-1}\right)\right), \quad N \rightarrow \infty,
$$

holds locally uniformly in $\mathbb{C} \backslash\left(\operatorname{supp} \mu_{\alpha, 1} \cup \operatorname{supp} \mu_{\alpha, 2}\right)$.

Since we take $P_{n, m}$ monic, constant $r_{1}$ can be deduced from the normalization

$$
\lim _{z \rightarrow \infty}\left(g_{\alpha_{N}, 1}(z)-\frac{2 z^{3}}{3}+\log z\right)=r_{1} .
$$

Theorem 2.8. For $n, m$ large enough such that $\alpha_{N} \stackrel{\text { def }}{=} n / N \rightarrow \alpha \in(0,1 / 2) \backslash\left\{\alpha_{c}\right\}$, $N=n+m$, the polynomial $A_{n, m}$ exists, and for an appropriate constant $r_{2}=r_{\alpha_{N}, 2}$,

$$
2 \pi i A_{n, m}(z)=f_{12}(z) e^{N\left(g_{\alpha_{N}, 2}(z)+\frac{z^{3}}{3}-r_{2}\right)}\left(1+\mathcal{O}\left(N^{-1}\right)\right), \quad N \rightarrow \infty,
$$

locally uniformly in $\mathbb{C} \backslash\left(\operatorname{supp} \mu_{\alpha, 1} \cup \operatorname{supp} \mu_{\alpha, 3}\right)$. 
As we can infer from Theorem 2.5 the asymptotic formula for $B_{n, m}$ is a little bit more involved, and requires introducing a sub-domain of the plane, that we denote by $\Omega_{\alpha}$ :

(i) (subcritical regime) if $0<\alpha<\alpha_{c}$, then $\Omega_{\alpha}=\emptyset$;

(ii) (supercritical regime) for $\alpha>\alpha_{c}$, then $\Omega_{\alpha}$ is the bounded connected component of $\mathbb{H}_{-} \backslash E_{\alpha}$.

Theorem 2.9. For $n, m$ large enough such that $\alpha_{N} \stackrel{\text { def }}{=} n / N \rightarrow \alpha \in(0,1 / 2) \backslash\left\{\alpha_{c}\right\}$, $N=n+m$, the polynomial $B_{n, m}$ exists, and for an appropriate constant $r_{3}=r_{\alpha_{N}, 3}$,

$$
\begin{aligned}
& 2 \pi i B_{n, m}(z)= \\
& \begin{cases}f_{13}(z) e^{N\left(g_{\alpha_{N}, 3}(z)+\frac{1}{3} z^{3}-r_{3}\right)}(1+\mathcal{O}(1 / N)), & \text { loc. uniformly in } \mathbb{C} \backslash\left(\Omega_{\alpha} \cup \Delta_{2}\right), \\
-f_{12}(z) e^{N\left(g_{\alpha_{N}, 2}(z)+\frac{1}{3} z^{3}-r_{3}\right)}(1+\mathcal{O}(1 / N)), & \text { loc. uniformly in } \Omega_{\alpha} .\end{cases}
\end{aligned}
$$

Remark 2.10. In Theorems 2.7 2.9 the functions $f_{1 j}$ are defined on the Riemann surface $\mathcal{R}$ of the spectral curve (2.7) for the limiting value $\alpha$, so they do not depend on $\alpha_{N}$. As it was mentioned earlier, they can be constructed in terms of certain abelian integral on $\mathcal{R}$. Taking advantage of the fact that $\mathcal{R}$ is of genus 0 , we could in theory use the rational parametrization of $\mathcal{R}$ to give a more explicit representation of $f_{1 j}$ 's. However attractive, a practical implementation of this program requires finding this rational parametrization, and in virtue of the relatively high total degree of (2.7) this turned out to be a formidable task that we were not able to complete.

\section{SpeCtral CURVE AND AUXiLiary FUnCTIONS}

In this section, for convenience of the reader, we summarize some properties of the solutions of the spectral curve (2.7)-2.9) for $\alpha \in[0,1 / 2]$, or equivalently, for

$$
\tau=\alpha(1-\alpha) \in[0,1 / 4],
$$

and introduce some related auxiliary functions. It was shown in 37 that the algebraic equation (2.7) with coefficients given by $(2.8)$ and $(2.9)$, has four branch points and a double point. Two of the branch points, $a_{1}<b_{1}$, are real and the other two form a complex conjugate pair: $b_{2}=\overline{a_{2}}, \operatorname{Im} a_{2}<0$. These are the same $a_{j}$ 's and $b_{j}$ 's used in the previous section to denote the end points of the support of the components $\mu_{k}$ of the vector critical measure $\vec{\mu}_{\alpha}$. For $\tau \neq 1 / 12$ all these points are distinct, while when $\tau=1 / 12$, the double point and one of the real branch points of 2.7) coalesce.

The three solutions $\xi_{1}, \xi_{2}, \xi_{3}$ of (2.7) are determined by their asymptotic expansion at infinity,

$$
\begin{aligned}
& \xi_{1}(z)=2 z^{2}-\frac{1}{z}+\mathcal{O}\left(z^{-2}\right), \\
& \xi_{2}(z)=-z^{2}+\frac{\alpha}{z}+\mathcal{O}\left(z^{-2}\right), \\
& \xi_{3}(z)=-z^{2}+\frac{1-\alpha}{z}+\mathcal{O}\left(z^{-2}\right),
\end{aligned}
$$

and by their domain of definition: $\xi_{1}$ is defined on $\mathbb{C} \backslash\left(\Delta_{1} \cup \Delta_{2}\right), \xi_{2}$ on $\mathbb{C} \backslash$ $\left(\Delta_{1} \cup \Delta_{3}\right)$, and $\xi_{3}$ on $\mathbb{C} \backslash\left(\Delta_{2} \cup \Delta_{3}\right)$, images of the respective sheets $\mathcal{R}_{j}$ onto $\mathbb{C}$ by canonical projection, see Figure 7 Each $\xi_{j}$ is a meromorphic function in its domain of definition, with the only pole at infinity. 


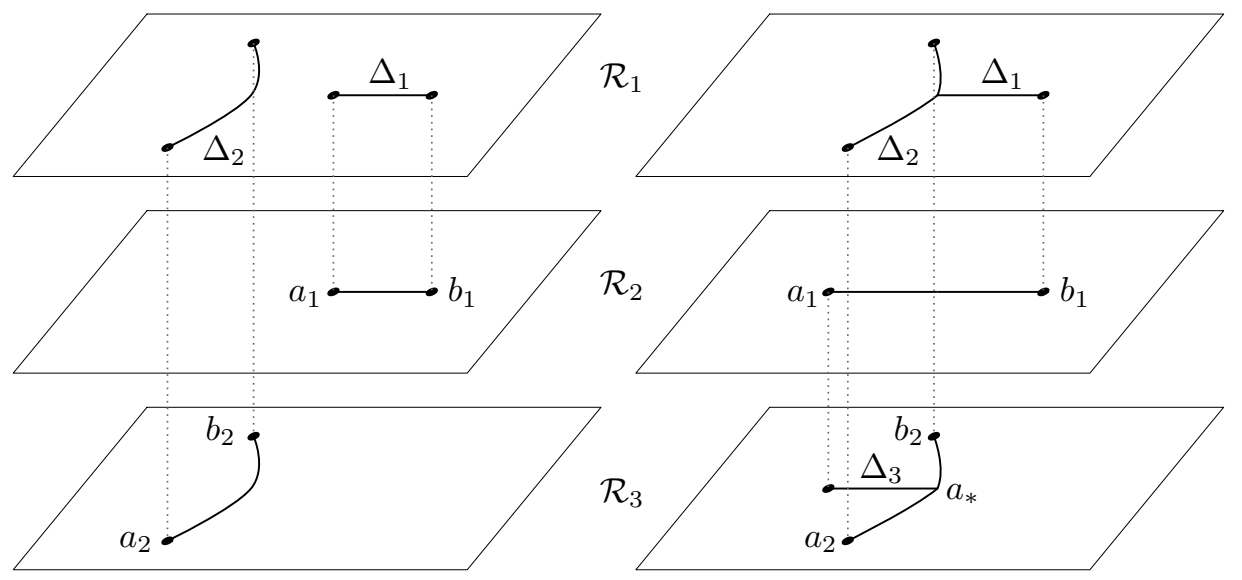

FiguRE 7. Sheet structure in the subcritical $0<\tau<\tau_{c}$ (left) and supercritical $\tau_{c}<\tau<1 / 4$ (right) regimes (reproduced from 37$]$ ).

Let us discuss some notation that we will use frequently. According to a standard convention, any orientation of a piece-wise smooth arc on $\mathbb{C}$ induces the left (denoted by the subscript "+") and right (with the subscript "-") boundary values of functions defined in a neighborhood of such an arc.

For what comes next, recall the definition of the upper and lower half planes $\mathbb{H}_{+}$ and $\mathbb{H}_{-}$in 2.10 . In addition, for $\varepsilon>0$ and $a \in \mathbb{C}$, set $B(a, \varepsilon) \stackrel{\text { def }}{=}\{z \in \mathbb{C}:|z-a|<$ $\varepsilon\}$. If $\mathcal{U}$ is a domain on $\mathbb{C}$, then $\partial \mathcal{U}$ stands for its boundary.

Moreover, if $\Gamma$ is a finite union of smooth arcs, then $\stackrel{\circ}{\Gamma}$ the union of all $a \in \Gamma$ for which there exists a small $\varepsilon>0$ such that $B(a, \varepsilon) \cap \Gamma$ is a smooth Jordan arc. Loosely speaking, $\stackrel{\circ}{\Gamma}$ is $\Gamma$ with all its end points, points of self-intersection and discontinuities of the derivative removed.

Now we can formulate the result that appears as Proposition 4.3 in [37], which will be the source of the needed identities and properties of $\xi_{j}$ 's:

Proposition 3.1. Let $\tau \in(0,1 / 4), \tau \neq \tau_{c} \stackrel{\text { def }}{=} \alpha_{c}\left(1-\alpha_{c}\right) \approx 0.1913565$. Then

(i) for $x \in \mathbb{R} \backslash\left(\Delta_{1} \cup \Delta_{2} \cup \Delta_{3}\right)$,

$$
\begin{aligned}
& \xi_{3}(x)<\xi_{2}(x)<\xi_{1}(x), \quad x<\min \left(a_{*}, a_{1}\right), \\
& \xi_{1}(x)<\xi_{2}(x)<\xi_{3}(x), \quad a_{*}<x<a_{1}, \quad \text { if } 0<\tau<\tau_{c}, \\
& \xi_{2}(x)<\xi_{3}(x)<\xi_{1}(x), \quad x>b_{*}, \\
& \xi_{2}(x)<\xi_{1}(x)<\xi_{3}(x), \quad b_{1}<x<b_{*}, \quad \text { if } \quad 0<\tau<1 / 12 \text {, } \\
& \xi_{3}(x)<\xi_{2}(x)<\xi_{1}(x), \quad b_{1}<x<b_{*}, \quad \text { if } 1 / 12<\tau<1 / 4 \text {. }
\end{aligned}
$$

Additionally,

$$
\begin{aligned}
& \xi_{2}\left(b_{*}\right)<\xi_{3}\left(b_{*}\right)=\xi_{1}\left(b_{*}\right), \quad \text { for } \quad 0<\tau<1 / 12, \\
& \xi_{2}\left(b_{*}\right)=\xi_{3}\left(b_{*}\right)<\xi_{1}\left(b_{*}\right), \quad \text { for } \quad 1 / 12<\tau<1 / 4 .
\end{aligned}
$$

(ii) on $\Delta_{1} \cup \Delta_{2} \cup \Delta_{3}$, 
- for $x \in \stackrel{\circ}{\Delta}_{1}:=\Delta_{1} \backslash\left\{\max \left(a_{1}, a_{*}\right), b_{1}\right\}$,

$$
\xi_{2}(x)=\overline{\xi_{1}(x)} \in \mathbb{C} \backslash \mathbb{R}, \quad \xi_{3}(x) \in \mathbb{R},
$$

and

$$
\xi_{1 \pm}(x)=\xi_{2 \mp}(x), \quad \xi_{3+}(x)=\xi_{3-}(x) .
$$

- for $z \in \stackrel{\circ}{\Delta}_{2}:=\Delta_{2} \backslash\left\{a_{2}, b_{2}, \max \left(a_{1}, a_{*}\right)\right\}$,

$$
\xi_{1 \pm}(z)=\xi_{3 \mp}(z), \quad \xi_{2+}(z)=\xi_{2-}(z) .
$$

- for $x \in \stackrel{\circ}{\Delta}_{3}:=\Delta_{3} \backslash\left\{a_{1}, a_{*}\right\}$ (when $\tau>\tau_{c}$ ),

$$
\xi_{2}(x)=\overline{\xi_{3}(x)} \in \mathbb{C} \backslash \mathbb{R}, \quad \xi_{1}(x) \in \mathbb{R},
$$

and

$$
\xi_{2 \pm}(z)=\xi_{3 \mp}(z), \quad \xi_{1+}(z)=\xi_{1-}(z) .
$$

Moreover,

$$
\begin{aligned}
& \xi_{1}\left(a_{1}\right)=\xi_{2}\left(a_{1}\right), \quad \text { if } \tau<\tau_{c}, \\
& \xi_{3}\left(a_{1}\right)=\xi_{2}\left(a_{1}\right), \quad \text { if } \tau>\tau_{c}, \\
& \xi_{1}\left(b_{1}\right)=\xi_{2}\left(b_{1}\right), \\
& \xi_{1}\left(a_{2}\right)=\xi_{3}\left(a_{2}\right) \quad \text { and } \quad \xi_{1}\left(b_{2}\right)=\xi_{3}\left(b_{2}\right) .
\end{aligned}
$$

Also, it follows from (3.1) and the structure of cuts $\Delta_{j}$ that $\xi_{j}$ have the following periods:

$$
\oint_{\Delta_{1} \cup \Delta_{2}} \xi_{1}=-2 \pi i, \quad \oint_{\Delta_{1} \cup \Delta_{3}} \xi_{2}=2 \pi i \alpha, \quad \oint_{\Delta_{2} \cup \Delta_{3}} \xi_{3}=2 \pi i(1-\alpha) .
$$

We define measures $\mu_{1}, \mu_{2}, \mu_{3}$ on $\Delta_{1}, \Delta_{2}, \Delta_{3}$, respectively, through the formulas

$$
\begin{aligned}
& d \mu_{1}(s) \stackrel{\text { def }}{=} \frac{1}{2 \pi i}\left(\xi_{1+}(s)-\xi_{2+}(s)\right) d s, \quad s \in \Delta_{1}, \\
& d \mu_{2}(s) \stackrel{\text { def }}{=} \frac{1}{2 \pi i}\left(\xi_{1+}(s)-\xi_{3+}(s)\right) d s, \quad s \in \Delta_{2},
\end{aligned}
$$

and

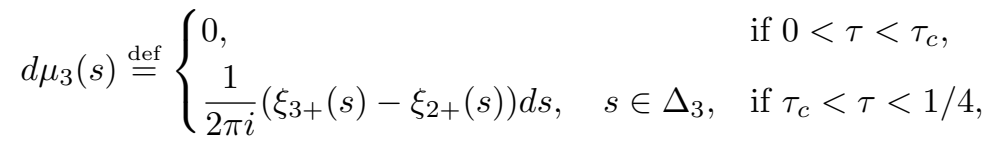

where $d s$ denotes the complex line element on the respective arc. As it was shown in [37. Proposition 3.4], these are bona fide positive measures, whose total masses satisfy the constraints 2.5 .

Furthermore, we define

$$
\begin{aligned}
& g_{1}(z) \stackrel{\text { def }}{=} \int_{b_{1}}^{z} \xi_{1}(s) d s+c_{1}, \quad z \in \mathbb{C} \backslash\left(\left(-\infty, b_{1}\right) \cup \Delta_{2}\right), \\
& g_{2}(z) \stackrel{\text { def }}{=} \int_{b_{1}}^{z} \xi_{2}(s) d s+c_{2}, \quad z \in \mathbb{C} \backslash\left(-\infty, b_{1}\right), \\
& g_{3}(z) \stackrel{\text { def }}{=} \int_{a_{*}}^{z} \xi_{3}(s) d s+c_{3}, \quad z \in \mathbb{C} \backslash\left(\left(-\infty, a_{*}\right) \cup \Delta_{2}\right),
\end{aligned}
$$


where for $g_{3}$ the path of integration emanates from $a_{*}$ in the direction $+\infty$. The constants are

$$
c_{1}=c_{2} \stackrel{\text { def }}{=} \int_{a_{*}}^{a_{2}}\left(\xi_{3-}(s)-\xi_{3+}(s)\right) d s, \quad c_{3} \stackrel{\text { def }}{=}-\int_{a_{*}}^{b_{1}} \xi_{1-}(s) d s .
$$

Notice that $c_{1}, c_{2} \in i \mathbb{R}_{-}$, which follows from an alternative representation

$$
c_{1}=-\pi i\left|\mu_{2}\right|=\int_{b_{2}}^{a_{*}}\left(\xi_{1+}(s)-\xi_{3+}(s)\right) d s,
$$

where $\mu_{2}$ is second component of the critical measure $\vec{\mu}$ (see (3.18)).

In their domains of definition, $\xi_{i}(\bar{z})=\overline{\xi_{i}(z)}, i=1,2,3$, and thus

$$
g_{i}(\bar{z})-c_{i}=\overline{g_{i}(z)-c_{i}}, \quad i=1,2,3
$$

were we again consider $z$ in the appropriate domain of definitions of the function.

Notice that from the asymptotic expansions in (3.1), we get the existence of constants $r_{j} \in \mathbb{C}$ such that

$$
\begin{aligned}
& g_{1}(z)=\frac{2}{3} z^{3}+r_{1}-\log z+\mathcal{O}\left(z^{-1}\right), \\
& g_{2}(z)=-\frac{1}{3} z^{3}+r_{2}+\alpha \log z+\mathcal{O}\left(z^{-1}\right), \quad z \rightarrow \infty . \\
& g_{3}(z)=-\frac{1}{3} z^{3}+r_{3}+(1-\alpha) \log z+\mathcal{O}\left(z^{-1}\right) .
\end{aligned}
$$

Because the functions $\xi_{1}$ and $\xi_{2}$ are real on the interval $\left(b_{1}, \infty\right)$ and $\xi_{3}$ is real on $\left(a_{*}, \infty\right)$, we surely have $r_{1}, r_{2}, r_{3} \in \mathbb{R}$.

Finally it will be convenient for the future to introduce additional functions, $\Phi_{j}$, closely related to $g_{j}$ 's. Namely, for $z \in \mathbb{C} \backslash\left(\left(-\infty, b_{1}\right] \cup \Delta_{2}\right)$, let

$$
\begin{aligned}
& \Phi_{1}(z) \stackrel{\text { def }}{=} \int_{b_{1}}^{z}\left(\xi_{1}(s)-\xi_{2}(s)\right) d s, \\
& \Phi_{2}(z) \stackrel{\text { def }}{=} \int_{b_{2}}^{z}\left(\xi_{1}(s)-\xi_{3}(s)\right) d s, \\
& \Phi_{3}(z) \stackrel{\text { def }}{=} \int_{\min \left(a_{1}, a_{*}\right)}^{z}\left(\xi_{3}(s)-\xi_{2}(s)\right) d s .
\end{aligned}
$$

The path of integration in the definition of $\Phi_{3}$ starts in $\mathbb{H}_{-}$, and if $a_{*}<a_{1}$ (i.e., if $\left.0<\tau<\tau_{c}\right)$, in the sector determined by $\left(a_{*},+\infty\right)$ and $\Delta_{2}$.

Using (3.17) and 3.20, we can see that for $z \in \mathbb{C} \backslash\left(\left(-\infty, b_{1}\right] \cup \Delta_{2}\right)$,

$$
\begin{aligned}
& \Phi_{1}(z)=g_{1}(z)-g_{2}(z), \\
& \Phi_{2}(z)=g_{1}(z)-g_{3}(z)+2 \pi i, \\
& \Phi_{3}(z)=g_{3}(z)-g_{2}(z)-2 \pi i \alpha+ \begin{cases}\int_{a_{*}}^{a_{1}}\left(\xi_{1}-\xi_{2}\right) d s+c_{2}, & 0<\tau<\tau_{c}, \\
0, & \tau_{c}<\tau<1 / 4 .\end{cases}
\end{aligned}
$$

\section{The Canonical quadratic Differential}

The study of the behavior of functions $\Phi_{j}$ 's, needed in what follows, relies heavily on the results and detailed analysis performed in our previous paper [37] of the trajectories of a canonical quadratic differential that we describe next. 
As it was established there (see Theorem 1.9), if

$$
Q(z) \stackrel{\text { def }}{=} \begin{cases}\xi_{2}(z)-\xi_{3}(z), & \text { on } \mathcal{R}_{1}, \\ \xi_{1}(z)-\xi_{3}(z), & \text { on } \mathcal{R}_{2}, \\ \xi_{1}(z)-\xi_{2}(z), & \text { on } \mathcal{R}_{3},\end{cases}
$$

then $Q^{2}$ extends to a meromorphic function on the Riemann surface $\mathcal{R}$ of the algebraic equation (2.7), and

$$
\varpi=-Q^{2}(z) d z^{2}
$$

is a meromorphic quadratic differential on $\mathcal{R}$ with poles only at the points at $\infty$. Finally, for $j=1,2,3$, each $\Delta_{j}=\operatorname{supp} \mu_{j}$ is an arc of trajectory of $\varpi$, satisfying

$$
\operatorname{Re} \int^{z} \sqrt{-\varpi}=\operatorname{Re} \int_{p}^{z} Q(s) d s=\text { const . }
$$

A trajectory $\gamma$ extending to a zero of $Q$ along at least one of its directions is called critical; in the case when it happens in both directions, we call this trajectory bounded (also finite or short), and unbounded (or infinite) otherwise. The union of finite and infinite critical trajectories is the critical graph of $\varpi$.

The parameter $\tau=\alpha(1-\alpha) \in[0,1 / 4]$ has transition values $\tau_{0}=1 / 12, \tau_{1}, \tau_{c}$ and $\tau_{2}$, which satisfy

$$
0<\tau_{0}<\tau_{1}<\tau_{c}<\tau_{2}<\frac{1}{4}
$$

and determine the global structure of trajectories of the quadratic differential $\varpi$ defined in (4.1). According to 37, at $\tau=\tau_{0}$ the double point (node) of $\mathcal{R}$ collides with the branch point $b_{1}$ transitioning from $\mathcal{R}_{2}$ to $\mathcal{R}_{1}$; at $\tau=\tau_{1}$, a critical trajectory connecting $a_{2}^{(1)}$ and $b_{2}^{(1)}$ on $\mathcal{R}_{1}$ hits the branch point $a_{1}^{(1)}$, while at $\tau=\tau_{c}$ the same happens on $\mathcal{R}_{2}$, now for $a_{2}^{(2)}, b_{2}^{(2)}$ and $a_{1}^{(2)}$. Finally, for $\tau=\tau_{2}$, the point $a_{*}$ collides with the critical trajectory connecting $a_{2}^{(1)}$ and $b_{2}^{(1)}$ on $\mathcal{R}_{1}$; at this moment, there are short trajectories connecting $a_{1}^{(1)}$ with $a_{2}^{(1)}$ and $b_{2}^{(1)}$.

As described in [37, the critical graph of $\varpi$ is topologically the same for $\tau$ in each of the intervals determined by consecutive transition values $0, \tau_{0}, \tau_{1}, \tau_{c}, \tau_{2}, 1 / 4$, and it undergoes a phase transition when $\tau$ moves from one of these intervals to the next one.

Of particular relevance for us here are the trajectories of $\varpi$ on $\mathcal{R}_{1}$, so in Figures 8 9 we reproduce these relevant illustrations as provided in 37 , Figures 10,12,15,19,21]

We need to describe some domains of positivity of functions $\operatorname{Re} \Phi_{j}$. Notice that by (3.23)-(3.25), the level curves of $\operatorname{Re} \Phi_{j}$ coincide with trajectories of the quadratic differential $\varpi$ on the sheet $\mathcal{R}_{4-j}, j=1,2,3$, which establishes the connection with the topology of the critical graph of $\varpi$.

As it follows from the detailed analysis of the global structure of the trajectories performed in 37, Section 4.5], for $1 / 12<\tau<1 / 4$ there exists an arc of trajectory $\gamma_{0}$ emanating from $b_{1}^{(1)}$ on the lower half-plane (on the sheet $\mathcal{R}_{1}$ ), and crossing the real line at a point $x_{0} \in \Delta_{1}$. Let us define

$$
x_{*} \stackrel{\text { def }}{=} \begin{cases}b_{1}, & 0<\tau \leq 1 / 12, \\ x_{0}, & 1 / 12<\tau<1 / 4 .\end{cases}
$$

The next lemma is complementary to Proposition 3.1 

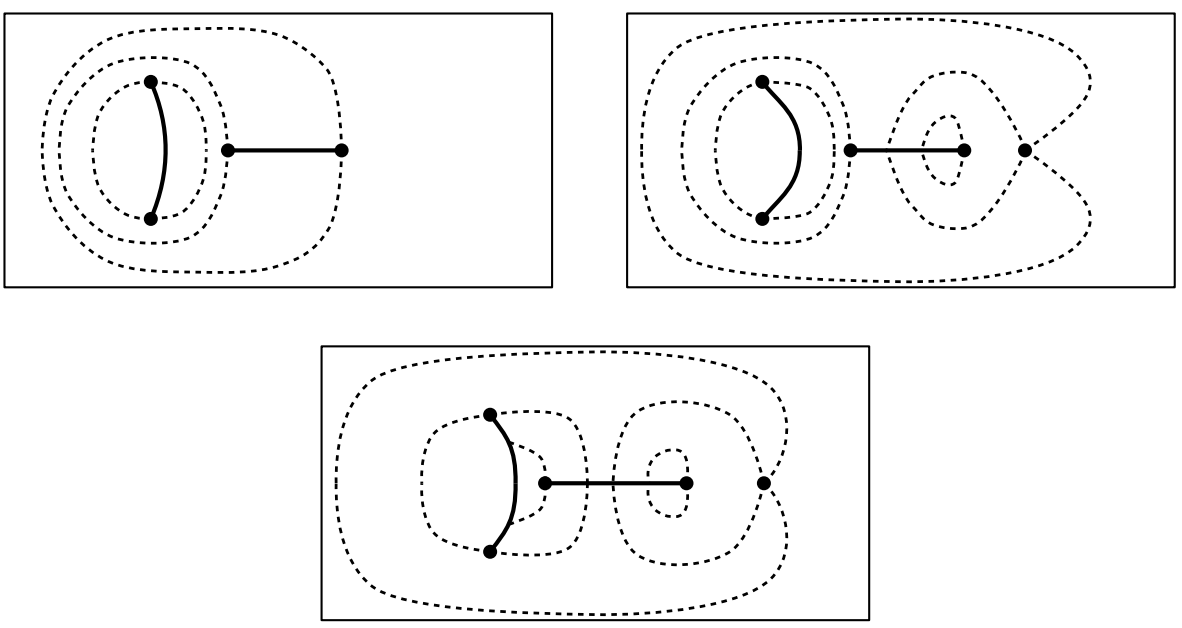

Figure 8. Critical graph of $\varpi$ on the first sheet $\mathcal{R}_{1}$, corresponding to the intervals $0<\tau<\tau_{0}$ (top left frame), $\tau_{0}<\tau<\tau_{1}$ (top right frame) and $\tau_{1}<\tau<\tau_{c}$ (bottom frame). The dashed lines represent the critical trajectories, whereas the solid lines are the branch cuts connecting $\mathcal{R}_{1}$ to the remaining sheets, which are not displayed.
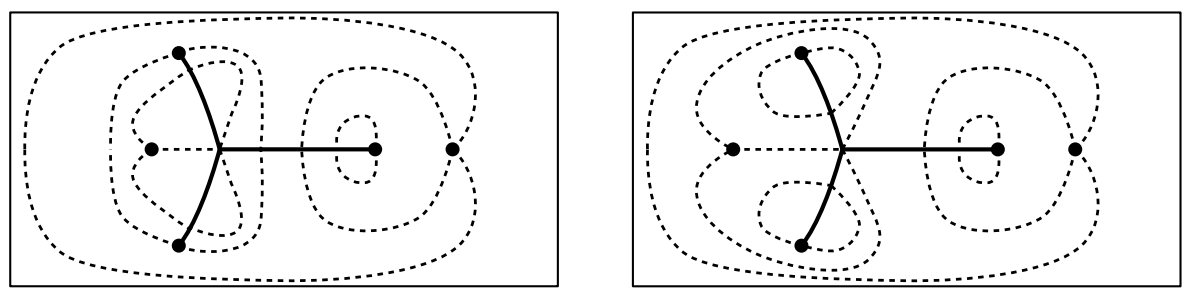

Figure 9. Critical graph of $\varpi$ on the first sheet $\mathcal{R}_{1}$, corresponding to the intervals $\tau_{c}<\tau<\tau_{2}$ (left frame) and $\tau_{2}<\tau<1 / 4$ (right frame). The dashed lines represent the critical trajectories, whereas the solid lines are the branch cuts connecting $\mathcal{R}_{1}$ to the remaining sheets, which are not displayed.

Lemma 4.1. If $\tau<1 / 12$, then

$$
\operatorname{Re} \xi_{2 \pm}(x)<\xi_{3}(x), \quad x>a_{*} .
$$

If $\tau>1 / 12$, then there exists a value $\zeta_{*} \in\left(x_{*}, b_{1}\right)$ such that

$$
\operatorname{Re} \xi_{2 \pm}(x)<\xi_{3}(x), \quad x \in\left(a_{*}, \zeta_{*}\right) \cup\left(b_{*},+\infty\right)
$$

and

$$
\operatorname{Re} \xi_{2 \pm}(x)>\xi_{3}(x), \quad x \in\left(\zeta_{*}, b_{*}\right) .
$$

Proof. By (3.3)-(3.6), we only need to establish the inequalities on $\Delta_{1}$.

Recall that $\xi_{1}, \xi_{2}$ and $\xi_{3}$ are the solutions of the cubic equation $\xi^{3}-R(z) \xi+$ $D(z)=0$ in 2.7) and that $\xi_{2 \pm}=\xi_{1 \mp}$ on $\Delta_{1}$, whereas $\xi_{3}$ is analytic across $\Delta_{1}$. Consequently, by (3.8)-3.9), if $\xi_{3}\left(\zeta_{*}\right)=\operatorname{Re} \xi_{2 \pm}\left(\zeta_{*}\right)$ for some value $\zeta_{*} \in \Delta_{1}$, then

$$
0=\xi_{1+}\left(\zeta_{*}\right)+\xi_{2+}\left(\zeta_{*}\right)+\xi_{3}\left(\zeta_{*}\right)=3 \xi_{3}\left(\zeta_{*}\right),
$$


and consequently $D\left(\zeta_{*}\right)=\xi_{1+}\left(\zeta_{*}\right) \xi_{2+}\left(\zeta_{*}\right) \xi_{3}\left(\zeta_{*}\right)=0$. From [37, Proposition 4.2] we know that $D$ has no zeros on $\Delta_{1}$ if $\tau<1 / 12$, and has exactly one zero on $\Delta_{1}$ otherwise, which thus has to be $\zeta_{*}$. In other words, $\xi_{2 \pm}-\xi_{3}$ does not change sign on $\Delta_{1}$ (if $\tau<1 / 12$ ) or otherwise, it can change sign at most once, and it has to be at the zero $\zeta_{*}$ of $D$.

Case $\tau<1 / 12$ then trivially follows from 3.3 and 3.13 ; so, let us consider $1 / 12<\tau<\tau_{c}$.

By the definition of the trajectory $\gamma_{0}$ emanating from $b_{1}$ on the lower half plane in the first sheet,

$$
0=\operatorname{Re} \int_{\gamma_{0}} \sqrt{-\varpi}=\int_{b_{1}}^{x_{*}}\left(\operatorname{Re} \xi_{2-}(x)-\xi_{3}(x)\right) d x .
$$

Hence, $\operatorname{Re} \xi_{2-}(x)-\xi_{3}(x)$ has to change sign on $\left(x_{*}, b_{1}\right)$, thus $\zeta_{*}>x_{*}$, so consequently $\operatorname{Re} \xi_{2-}(x)-\xi_{3}(x)$ has constant sign on $\Delta_{1} \backslash\left[x_{*}, b_{1}\right]$.

It remains to use (3.3) and (3.13): when combined these equations give us that for $\tau<\tau_{c}$ we have $\xi_{2}<\xi_{3}$ immediately to the right of $a_{1}$, so that by continuity of the $\xi_{j}$ 's in both $z$ and $\tau$ we conclude the proof.

As a preparatory step for the study of positivity of $\operatorname{Re} \Phi_{3}$, let us examine the boundary values of $\Phi_{3}$ on subsets of $\mathbb{R}$ from the lower half plane.

Assume first $\tau<\tau_{c}$. Combining 3.10, 3.20 and 3.25), for $x<a_{*}$,

$$
\begin{aligned}
\Phi_{3-}(x) & =\int_{a_{*}}^{a_{2}}\left(\xi_{3-}(s)-\xi_{3+}(s)\right) d s+\int_{a_{*}}^{x}\left(\xi_{3}(s)-\xi_{2}(s)\right) d s \\
& =c_{2}+\int_{a_{*}}^{x}\left(\xi_{3}(s)-\xi_{2}(s)\right) d s,
\end{aligned}
$$

so that

$$
\operatorname{Re} \Phi_{3-}(x)=\int_{a_{*}}^{x}\left(\xi_{3}(s)-\xi_{2}(s)\right) d s>0, \quad x<a_{*},
$$

where we have used 3.2 . .

On the other hand, by $4.3-4.4$,

$$
\operatorname{Re} \Phi_{3-}(x)=\int_{a_{*}}^{x}\left(\xi_{3}(s)-\xi_{2-}(s)\right) d s>0, \quad a_{*}<x \leq \zeta_{*},
$$

where as we have seen, $a_{1}<x_{*}<\zeta_{*} \leq b_{1}$.

Let now $\tau>\tau_{c}$, so that

$$
\Phi_{3}(z)=\int_{a_{1}}^{z}\left(\xi_{3}(s)-\xi_{2}(s)\right) d s,
$$

with the path of integration starting in $\mathbb{H}_{-}$. In particular, using 3.2

$$
\Phi_{3-}(x)=\int_{a_{1}}^{x}\left(\xi_{3}(s)-\xi_{2}(s)\right) d s>0, \quad x<a_{1} .
$$

Furthermore, for $x \in\left(a_{1}, a_{*}\right)=\stackrel{\circ}{\Delta}_{3}$, we now use 3.6 and get

$$
\Phi_{3-}(x)=\int_{a_{1}}^{x}\left(\xi_{3-}(s)-\xi_{2-}(s)\right) d s=\int_{a_{1}}^{x}\left(\xi_{3-}(s)-\overline{\xi_{3-}(s)}\right) d s,
$$

so that $\operatorname{Re} \Phi_{3-}(x)=0$ for $x \in\left[a_{1}, a_{*}\right]$. 
On the other hand, for $x \in\left(a_{*}, \zeta_{*}\right]$,

$$
\begin{array}{r}
\Phi_{3-}(x)=\int_{a_{1}}^{a_{*}}\left(\xi_{3-}(s)-\overline{\xi_{3-}(s)}\right) d s+\int_{a_{*}}^{a_{2}}\left(\xi_{3+}(s)-\xi_{3-}(s)\right) d s \\
+\int_{a_{*}}^{x}\left(\xi_{3}(s)-\xi_{2-}(s)\right) d s \\
=\int_{a_{1}}^{a_{*}}\left(\xi_{3-}(s)-\overline{\xi_{3-}(s)}\right) d s-c_{2}+\int_{a_{*}}^{x}\left(\xi_{3}(s)-\xi_{2-}(s)\right) d s,
\end{array}
$$

so that 4.6 holds also in this case.

Our findings are summarized as

$$
\operatorname{Re} \Phi_{3-}(x) \begin{cases}>0, & x<\min \left(a_{1}, a_{*}\right) \text { or } a_{*}<x \leq \zeta_{*}, \\ =0, & a_{1}<x<a_{*} \text { and } \tau>\tau_{c} .\end{cases}
$$

Now we are ready to prove a statement that will be important for our asymptotic analysis. We will be interested in what happens in the lower half plane $\mathbb{H}_{-}$, so we introduce

$$
\Omega_{-} \stackrel{\text { def }}{=}\left\{z \in \mathbb{H}_{-} \backslash \Delta_{2}: \operatorname{Re} \Phi_{3}(z)<0\right\}, \quad \Omega_{+} \stackrel{\text { def }}{=}\left\{z \in \mathbb{H}_{-} \backslash \Delta_{2}: \operatorname{Re} \Phi_{3}(z)>0\right\} .
$$

Proposition 4.2. The structure of $\Omega_{-}$is as follows:

(i) For $0<\tau<\tau_{c}, \Omega_{-}=\emptyset$.

(ii) For $\tau_{c}<\tau<\tau_{2}, \Omega_{-}$is an open set with two connected components, whose boundaries intersect at the same subarc $\left(a_{*}, z_{B}\right)$ of $\Delta_{2}$, where $z_{B} \in \Delta_{2}$, $z_{B} \neq a_{2}$. One of these two components contains also the interval $\left(a_{1}, a_{*}\right)$ on its boundary, and the boundary of the other component intersects the real axis only at $a_{*}$.

(iii) For $\tau_{2}<\tau<1 / 4, \Omega_{-}$is an open connected set whose boundary contains both the arc $\Delta_{2} \cap \mathbb{H}_{-}$and the interval $\left(a_{1}, a_{*}\right)$.

Furthermore, there exists an unbounded domain $\mathcal{V} \subset \mathbb{H}_{-}$, with $a_{2}$ on its boundary and extending to $\infty$ along the direction determined by the angle $-2 \pi / 3$, such that

$$
\operatorname{Re} \Phi_{1}(z)>0, \quad \operatorname{Re} \Phi_{2}(z)>0, \quad z \in \mathcal{V} .
$$

Proof. We start with some basic considerations. Because Re $\Phi_{3}$ is harmonic on $\mathbb{H}_{-} \backslash \Delta_{2}$, the boundary of any connected component of $\Omega_{ \pm}$can only emanate from $\mathbb{R}, \Delta_{2}$ or $\infty$. Furthermore, any level line of $\operatorname{Re} \Phi_{3}$ is an arc of trajectory of the quadratic differential 4.1) on $\mathcal{R}_{1}$. A common feature of such trajectories is that they are closed arcs near $\infty$ (see Figures 8 9), so combined with the inequality (4.8) as $x \rightarrow-\infty$, we conclude that a neighborhood of $\infty$ belongs to $\Omega_{+}$or, in other words, $\Omega_{-}$is bounded.

Also, observe that the boundary of $\Omega_{-}$does not contain points on the interval $\left(-\infty, \min \left\{a_{1}, a_{*}\right\}\right)$. This is true because if $\tilde{b}$ is the smallest such point, then $\operatorname{Re} \Phi_{3}$ is harmonic immediately below to $\tilde{b}$, so we must have $\tilde{b} \in \partial \Omega_{-} \cap \partial \Omega_{+}$and thus $\operatorname{Re} \Phi_{3_{-}}(\tilde{b})=0$, which is in contradiction with 4.8 .

We claim that the boundary of $\Omega_{-}$does not contain points on $\left(a_{*}, \infty\right)$ either. Indeed, to the contrary, suppose that $b$ is such a point. We can assume $b$ to be the right-most of such points. Consequently $\Phi_{3-}(b)=0$ and any $x>b$ belongs to $\bar{\Omega}_{+}$. Take the smallest possible $a \geq a_{*}$ such that $[a, b] \subset \partial \Omega_{-}$. If $a=a_{*}$ then $\operatorname{Re} \Phi_{3-}(a)=0$ (the value understood when we approach $a_{*}$ from the sector determined by $\Delta_{2} \cap \mathbb{H}_{-}$and $\left.\left(a_{*},+\infty\right)\right)$, whereas if $a>a_{*}$ then again $\operatorname{Re} \Phi_{3-}\left(a_{*}\right)=$ 
0 , but now because $\operatorname{Re} \Phi_{3}$ is harmonic in a neighborhood immediately below $a$. In either case, we can certainly write

$$
0=\operatorname{Re} \Phi_{3-}(b)-\operatorname{Re} \Phi_{3-}(a)=\operatorname{Re} \int_{a}^{b}\left(\xi_{3-}(s)-\xi_{2-}(s)\right) d s,
$$

so $\xi_{3-}-\xi_{2-}$ must change sign on $(a, b)$. In virtue of 4.3 -4.5 , we must have at least one of the points $b_{*}$ and $\zeta_{*}$ in $(a, b)$, so certainly $b \geq \zeta_{*}$. Now, by construction, $\bar{\Omega}_{+}$is to the right-hand side of $b$, so $\xi_{3-}-\xi_{2-}$ must be positive for values slightly larger than $b$. Again using (4.4)-4.5), we thus get that actually $b>b_{*}$.

Let $\gamma$ be the arc of $\partial \Omega_{-}$that emanates from $b$, oriented outwards of $b$. With such orientation, $\Omega_{ \pm}$is on the \pm -side of $\gamma$. We follow the large behavior of $\gamma$, having in mind that $\gamma$ must be an arc of trajectory of $\varpi$ emanating from $\left(b_{*},+\infty\right)$. A quick inspection of the critical graphs in Figures 89 then shows that $\gamma$ must end up at a point $\tilde{b}<\min \left\{a_{*}, a_{1}\right\}$. Thus, keeping track of the orientation of $\gamma$, we then see that $\tilde{b} \in \partial \Omega_{-} \cap\left(-\infty, \min \left\{a_{*}, a_{1}\right\}\right)$, but we already know that this last intersection is empty.

As a conclusion of the observations above, the only possible real points on $\partial \Omega_{-}$ are on the interval $\left[\min \left\{a_{1}, a_{*}\right\}, a_{*}\right]$. 89

To verify (i)-(iii), we again use the structure of trajectories shown in Figures

For (i), notice first that $\left[\min \left\{a_{1}, a_{*}\right\}, a_{*}\right]=\left\{a_{*}\right\}$. Observing the structure displayed in Figure 8, we get that $\operatorname{Re} \Phi_{3}(z)$ does not change sign on the immediate vicinity of $a_{*}$, so $a_{*} \notin \partial \Omega_{-}$, and also that any possible boundary component of $\Omega_{-}$ in $\mathbb{H}_{-}$must intersect the real axis, which we showed that cannot occur away from $a_{*}$.

For (ii) and (iii), we observe that $\left[\min \left\{a_{1}, a_{*}\right\}, a_{*}\right]$ and the only regions on $\mathbb{H}_{-}$ that contain trajectories which do not intersect $\mathbb{R} \backslash\left[\min \left\{a_{1}, a_{*}\right\}, a_{*}\right]$ are the ones marked in gray in Figure 10 so $\Omega_{-}$must be contained in these regions. A simple analysis of the sign of $\operatorname{Re} \Phi_{3}$, making use of (4.8), then shows that $\Omega_{-}$actually coincides with these marked sectors. This concludes the proof of (ii) and (iii).

Finally, for every value of $\tau$ there is a half-plane canonical domain on $\mathcal{R}_{2}$ and a half-plane canonical domain on $\mathcal{R}_{3}$, both extending to $\infty$ in the asymptotic direction $e^{-2 \pi / 3}$, containing $a_{2}$ on its boundary. Take as $\mathcal{V}$ the (non-empty) intersection of the projection of these domains onto $\mathbb{C}$. Functions $\operatorname{Re} \Phi_{2}$ and $\operatorname{Re} \Phi_{1}$ do not change sign in $\mathcal{V}$, and by $3.22,3.26$ ) and (3.27),

$$
\Phi_{1}(z)=z^{3}+\mathcal{O}(1), \quad \Phi_{2}(z)=z^{3}+\mathcal{O}(1), \quad z \in \mathcal{V}, z \rightarrow \infty,
$$

from where 4.9 follows.

For $\tau \in\left(\tau_{c}, \tau_{2}\right)$, the boundary of $\Omega_{-}$consists of the interval $\left[a_{1}, a_{*}\right]$, an arc of trajectory $\gamma_{L}$ from $a_{1}$ to a point $a_{B} \in \Delta_{2}$, a second arc of trajectory $\gamma_{R}$ from $a_{B}$ to $a_{*}$ and a third $\operatorname{arc}\left(a_{B}, a_{*}\right) \subset \Delta_{2}$ connecting $a_{B}$ to $a_{*}$. It is also a consequence of the analysis in 37 that the arc of trajectory $\gamma_{R}$ is the analytic extension of $\Delta_{2} \cap \mathbb{H}_{+}$ to the lower half plane. These quantities are displayed in Figure 11, left frame.

When $\tau>\tau_{c}$, the boundary of $\Omega_{-}$consists of $\Delta_{2} \cap \mathbb{H}_{-}$, the interval $\left[a_{1}, a_{*}\right]$ and an $\operatorname{arc} \gamma_{R}$ joining $a_{1}$ and $a_{*}$, the latter being the analytic extension of $\Delta_{2} \cap \mathbb{H}_{+}$to the lower half plane. These are displayed in Figure 11, right frame. 

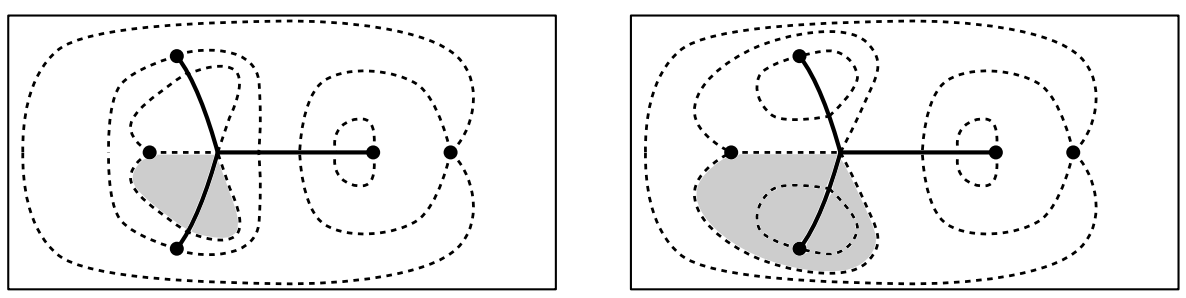

Figure 10 . The set $\Omega_{-}$is displayed in gray, together with the trajectories of $\varpi$ on the first sheet $\mathcal{R}_{1}$. The left frame corresponds to $\tau_{c}<\tau<\tau_{2}$ and the right frame corresponds to $\tau_{2}<\tau<1 / 2$.
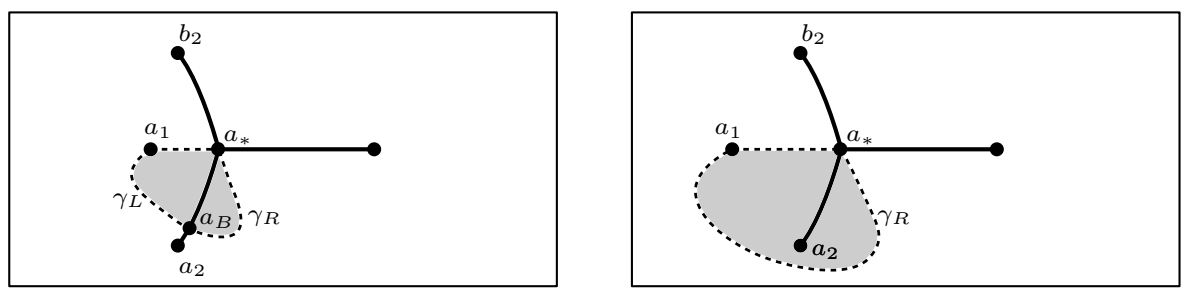

Figure 11. For the cases $\tau_{c}<\tau<\tau_{2}$ (left frame) and $\tau_{2}<\tau<$ $1 / 4$ (right frame) the set $\Omega_{-}$is displayed in gray, together with the newly introduced $\operatorname{arcs} \gamma_{L}$ and $\gamma_{B}$ and the point $a_{B}$.

Using the notation just introduced, we define the open set $\Omega_{\alpha} \subset \mathbb{H}_{-}$and the continuum $E_{\alpha} \subset \mathbb{C}$ that will play an important role in the asymptotic analysis of $B_{n, m}$ :

Definition 4.3. (i) If $\tau \in\left(0, \tau_{c}\right)$, then $\Omega_{\alpha} \stackrel{\text { def }}{=} \emptyset$ and $E_{\alpha} \stackrel{\text { def }}{=} \Delta_{2}$. Notice that on $E_{\alpha}$,

$$
\operatorname{Re}\left(\Phi_{3+}-\Phi_{3-}\right)(z)=0
$$

(ii) If $\tau \in\left(\tau_{c}, \tau_{2}\right)$, then

$$
E_{\alpha} \stackrel{\text { def }}{=} \gamma_{L} \cup \gamma_{R} \cup\left(a_{2}, a_{B}\right) \cup\left(\Delta_{2} \cap \mathbb{H}_{+}\right),
$$

where $\left(a_{2}, a_{B}\right)$ denotes the arc of $\Delta_{2}$ connecting $a_{2}$ with $a_{B}$. On $\left(a_{2}, a_{B}\right)$, identity (4.10) holds, while on $\gamma_{L} \cup \gamma_{R}, \operatorname{Re} \Phi_{3}(z)=0$. Notice also that $\overline{\gamma_{R} \cup\left(\Delta_{2} \cap \mathbb{H}_{+}\right)}$is an analytic arc joining $a_{B}$ and $b_{2}$.

Furthermore, $\Omega_{\alpha}$ is the bounded component of $\mathbb{C} \backslash\left(\mathbb{R} \cup \gamma_{L} \cup \gamma_{R}\right)$.

(iii) For $\tau \in\left(\tau_{2}, 1 / 4\right)$, the set $E_{\alpha} \stackrel{\text { def }}{=} \gamma_{L} \cup\left(\Delta_{2} \cap \mathbb{H}_{+}\right)$is a single analytic arc from $b_{2}$ to $a_{1}$, passing through $a_{*}$, and $\Omega_{\alpha}$ is the connected domain bounded by $\gamma_{L} \cup\left(a_{1}, a_{*}\right) \cup\left(\Delta_{2} \cap \mathbb{H}_{-}\right)$, that is, $\Omega_{\alpha}=\Omega_{-} \cup\left(a_{b}, a_{*}\right)$.

In any of the cases (i)-(iii) above, note also that

$$
E_{\alpha} \cap \mathbb{H}_{-}=\partial \Omega_{+} \cap \mathbb{H}_{-} .
$$

As the last step, we define the positive measure $\mu_{B}$ on the set $E_{\alpha}$ that will ultimately describe the zero distribution of $B_{n, m}$ as in Theorem 2.5 . 
Proposition 4.4. Let

$$
H(z) \stackrel{\text { def }}{=} \begin{cases}\operatorname{Re}\left(g_{2}(z)+\frac{1}{3} z^{3}-r_{1}\right), & z \in \Omega_{\alpha}, \\ \operatorname{Re}\left(g_{3}(z)+\frac{1}{3} z^{3}-r_{1}\right), & z \in \mathbb{C} \backslash\left(\Omega_{\alpha} \cup E_{\alpha}\right) .\end{cases}
$$

Then $H$ is harmonic in $\mathbb{C} \backslash E_{\alpha}$ and extends to a subharmonic function in $\mathbb{C}$. Moreover, there exists a positive measure $\mu_{B}, \operatorname{supp} \mu_{B}=E_{\alpha}$, such that

$$
H(z)=-U^{\mu_{B}}(z)+\operatorname{Re}\left(r_{3}-r_{1}\right), \quad z \in \mathbb{C},
$$

with constants $r_{1}, r_{3}$ defined in 3.22 . Measure $\mu_{B}$ satisfies $\left|\mu_{B}\right|=1-\alpha$, is absolutely continuous with respect to the arc-length measure, and

$$
\mu_{B}^{\prime}(s)= \begin{cases}\mu_{2}^{\prime}(s), & \text { for } s \in\left(\Delta_{2} \cap \mathbb{H}_{+}\right) \cup\left(\Delta_{2} \backslash \overline{\Omega_{\alpha}}\right), \\ \frac{1}{2 \pi i}\left(\xi_{2}(s)-\xi_{3}(s)\right), & \text { for } s \in \gamma_{L} \cup \gamma_{R} .\end{cases}
$$

Finally,

$$
\mu_{B}=\operatorname{Bal}\left(\mu_{2}-\mu_{3} ; E_{\alpha}\right) .
$$

Proof. The function $g_{3}$ is holomorphic on $\mathbb{C} \backslash\left(\left(-\infty, a_{*}\right) \cup \Delta_{2}\right)$, so we get that $H$ is harmonic on $\mathbb{C} \backslash\left(\left(-\infty, a_{*}\right) \cup \Delta_{2} \cup \Omega_{\alpha}\right)$. Also, $g_{3+}-g_{3-}$ is purely imaginary along $\left(-\infty, \min \left\{a_{1}, a_{*}\right\}\right)$ (see 6.6 below), so $H$ is harmonic across this interval as well. Furthermore, $g_{2+}=g_{3-}$ across $\Delta_{3}$ (see (6.9)), and this means that $H$ is harmonic across $\Delta_{3}$ as well. To conclude the harmonicity of $H$ on $\mathbb{C} \backslash E_{\alpha}$, it only remains to observe that, according to 3.19 , the function $g_{2}$ is holomorphic on the lower half plane.

Subharmonicity of $H$ in a neighborhood of $E_{\alpha} \cap \mathbb{H}_{-}$follows from the alternative representation

$$
H(z)=\max \left\{\operatorname{Re}\left(g_{2}(z)+\frac{1}{3} z^{3}-r_{1}\right), \operatorname{Re}\left(g_{3}(z)+\frac{1}{3} z^{3}-r_{1}\right)\right\},
$$

valid in a neighborhood of $E_{\alpha} \cap \mathbb{H}_{-}$, as assured by Proposition 4.2. Furthermore, using now (3.19) and (2.6) to write $H=-U^{\mu_{2}}+$ harmonic, we conclude that $H$ is subharmonic on a neighborhood of $E \cap \mathbb{H}_{+}$as well, and thus on the whole plane $\mathbb{C}$.

As a consequence of [44. Theorem II.3.3] there exists a positive measure $\mu_{B}$ on $E_{\alpha}$ and a harmonic function $h$ such that

$$
H(z)=-U^{\mu_{B}}(z)+h(z), \quad z \in \mathbb{C} .
$$

By 4.12 ,

$$
-C^{\mu_{B}}(z)+u^{\prime}(z)= \begin{cases}\xi_{2}(z)+z^{2}, & z \in \Omega_{\alpha}, \\ \xi_{3}(z)+z^{2}, & z \in \mathbb{C} \backslash\left(\Omega_{\alpha} \cup E_{\alpha}\right),\end{cases}
$$

where $u$ is an analytic function such that $\operatorname{Re} u=h$. Since by (3.1),

$$
\xi_{3}(z)+z^{2}=\frac{1-\alpha}{z}+\mathcal{O}\left(z^{-2}\right)
$$

we conclude that $\left|\mu_{B}\right|=1-\alpha$ and $h \equiv$ const.

The expression for $\mu_{B}^{\prime}$ is recovered using the Sokhotsky-Plemelj formula.

Finally, by 2.6.

$$
-C^{\mu_{2}-\mu_{3}}(z)=\xi_{3}(z)+z^{2}=-C^{\mu_{B}}(z), \quad z \in E_{\alpha},
$$

which is equivalent to 4.13. 


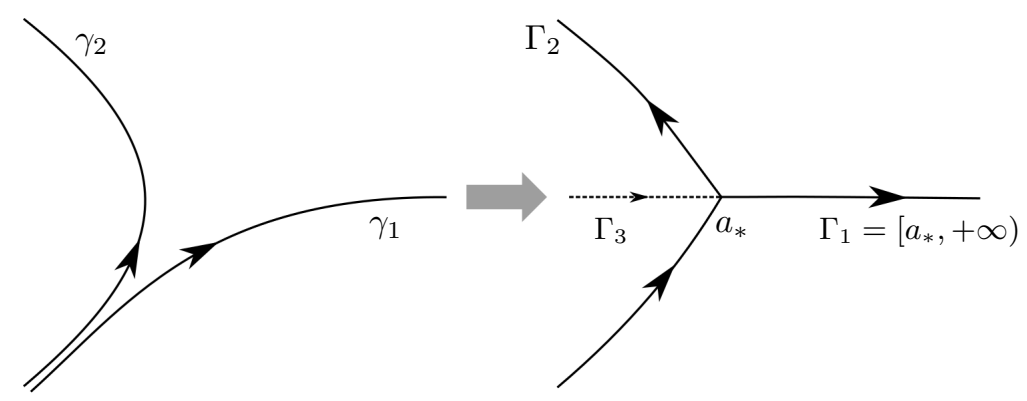

FiguRE 12. Orthogonality contours $\gamma_{1}, \gamma_{2}$ (left), and their deformation into a connected set $\Gamma_{1} \cup \Gamma_{2}$.

\section{The Riemann-Hilbert Formulation}

In this section, we characterize the polynomials defined by $(2.2)$ and $(2.3)$ in terms of a $3 \times 3$ non-commutative boundary value problem. It will be convenient to use the following matrix-related notation: for $a, b, c \in \mathbb{C}$,

$$
\operatorname{diag}(a, b, c) \stackrel{\text { def }}{=}\left(\begin{array}{ccc}
a & 0 & 0 \\
0 & b & 0 \\
0 & 0 & c
\end{array}\right) ;
$$

$\boldsymbol{e}_{1}, \boldsymbol{e}_{2}, \boldsymbol{e}_{3}$ are the column vectors of the $3 \times 3$ identity matrix $\boldsymbol{I}=\operatorname{diag}(1,1,1)$, and

$$
\boldsymbol{M}_{i j} \stackrel{\text { def }}{=} \boldsymbol{e}_{i} \boldsymbol{e}_{j}^{T} .
$$

Thus, $\boldsymbol{M}_{i j}$ is the $3 \times 3$ matrix whose only non-zero element is 1 in the position $(i, j)$. A straightforward consequence of this definition is that

$$
\boldsymbol{M}_{i j} \boldsymbol{M}_{a b}=\delta_{j a} \boldsymbol{M}_{i b},
$$

so that if $f, g$ are scalars, then for $j \neq a$,

$$
\left(\boldsymbol{I}+f \boldsymbol{M}_{i j}\right)\left(I+g \boldsymbol{M}_{a b}\right)=\boldsymbol{I}+f \boldsymbol{M}_{i j}+g \boldsymbol{M}_{a b} ;
$$

in particular, for $i \neq j$,

$$
\left(\boldsymbol{I}+f \boldsymbol{M}_{i j}\right)^{-1}=I-f \boldsymbol{M}_{i j} .
$$

Let two unbounded contours $\gamma_{1}$ and $\gamma_{2}$ extended to $\infty$ on their two ends along the directions determined by the angles $-2 \pi / 3$ and 0 , and $-2 \pi / 3$ and $2 \pi / 3$, respectively (see Figure 12, left; we assume them oriented as depicted there). For $n, m \in \mathbb{N} \cup\{0\}$, $N=n+m$, consider the following non-commutative Riemann-Hilbert problem (RHP): find a matrix-valued function $\boldsymbol{Y}: \mathbb{C} \backslash\left(\gamma_{1} \cup \gamma_{2}\right) \rightarrow \mathbb{C}^{3 \times 3}$, such that

- $\boldsymbol{Y}: \mathbb{C} \backslash\left(\gamma_{1} \cup \gamma_{2}\right) \rightarrow \mathbb{C}^{3 \times 3}$ is analytic;

- $\boldsymbol{Y}$ has continuous boundary values $\boldsymbol{Y}_{ \pm}$on $\gamma_{1} \cup \gamma_{2}$, and $\boldsymbol{Y}_{+}(z)=\boldsymbol{Y}_{-}(z) \boldsymbol{J}_{Y}(z)$ for $z \in \gamma_{1} \cup \gamma_{2}$, where

$$
\boldsymbol{J}_{Y}(z) \stackrel{\text { def }}{=} \begin{cases}I+e^{-N z^{3}} M_{12}, & z \in \gamma_{1} \\ I+e^{-N z^{3}} M_{13}, & z \in \gamma_{2} .\end{cases}
$$

- $\boldsymbol{Y}(z)=\left(I+\mathcal{O}\left(z^{-1}\right)\right) \operatorname{diag}\left(z^{N}, z^{-n}, z^{-m}\right), z \rightarrow \infty$. 


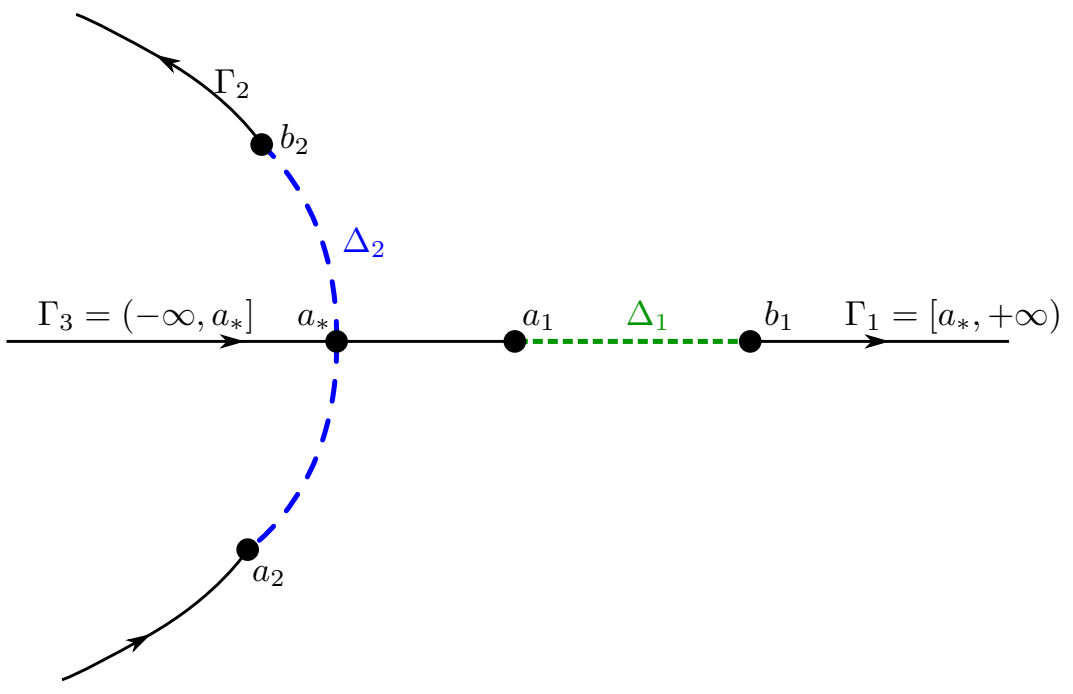

Figure 13. Pictorial representation of the sets $\Delta_{1}, \Delta_{2}, \Gamma_{1}, \Gamma_{2}$ and $\Gamma_{3}$ in the subcritical regime.

Obviously, matrix $\boldsymbol{Y}$ depends on $m$ and $n$ through the asymptotic condition at infinity, but we omit the explicit reference to $(m, n)$ from the notation whenever it cannot lead us into confusion.

Recall that for $\alpha \in[0,1 / 2]$, the typical structure of the support of the components of the critical vector measure $\vec{\mu}_{\alpha}=\left(\mu_{1}, \mu_{2}, \mu_{3}\right)$, described above, is depicted on Figure 3. More precisely, we consider three oriented sets, $\Delta_{j}=\operatorname{supp} \mu_{j}, j=1,2,3$, (which depend on $\alpha \in[0,1 / 2]$ ). Then $\Delta_{2}$ is a piece-wise analytic arc joining the complex-conjugate branch points $a_{2}, b_{2}, \operatorname{Im} a_{2}<0$, and oriented from $a_{2}$ to $b_{2}$. We denote $a_{*}=\Delta_{2} \cap \mathbb{R}$.

In the subcritical regime $\left(0<\tau<\tau_{c}\right), \Delta_{1}=\left[a_{1}, b_{1}\right] \subset \mathbb{R}$ oriented from $a_{1}$ to $b_{1}$, and set $\Delta_{3}=\emptyset$. In the supercritical regime $\left(\tau_{c}<\tau<1 / 4\right), \Delta_{1}=\left[a_{*}, b_{1}\right] \subset \mathbb{R}$ and $\Delta_{3}=\left[a_{1}, a_{*}\right] \subset \mathbb{R}$, both with the natural orientation.

Furthermore, let $\Gamma_{1}=\left[a_{*},+\infty\right) \supset \Delta_{1}, \Gamma_{3}=\left(-\infty, a_{*}\right] \supset \Delta_{3}$, and $\Gamma_{2}$ a piece-wise analytic curve extended to $\infty$ on its both ends along the directions determined by the angles $-2 \pi / 3$ and $2 \pi / 3$, and containing $\Delta_{2}$. We choose the orientation of $\Gamma_{j}$ 's consistent with those of $\Delta_{j}$; observe that $\Gamma_{1} \cup \Gamma_{2} \cup \Gamma_{3}$ is a connected set, see Figures 13 and 14

Since the orthogonality conditions in (2.3) are non-hermitian, we can deform the above mentioned contours $\gamma_{1}$ and $\gamma_{3}$ freely, preserving their asymptotic directions. In particular, we can make $\gamma_{2}$ coincide with $\Gamma_{2}$, while $\gamma_{1}$ will follow $\Gamma_{2} \cap \mathbb{H}_{-}$and $\Gamma_{1}$ (see Figure 12 , right). Thus, $\boldsymbol{Y}$ can be alternatively characterized by the following RHP:

- $\boldsymbol{Y}: \mathbb{C} \backslash \Gamma_{Y} \rightarrow \mathbb{C}^{3 \times 3}$ is analytic, where $\Gamma_{Y}=\Gamma_{1} \cup \Gamma_{2}$; 


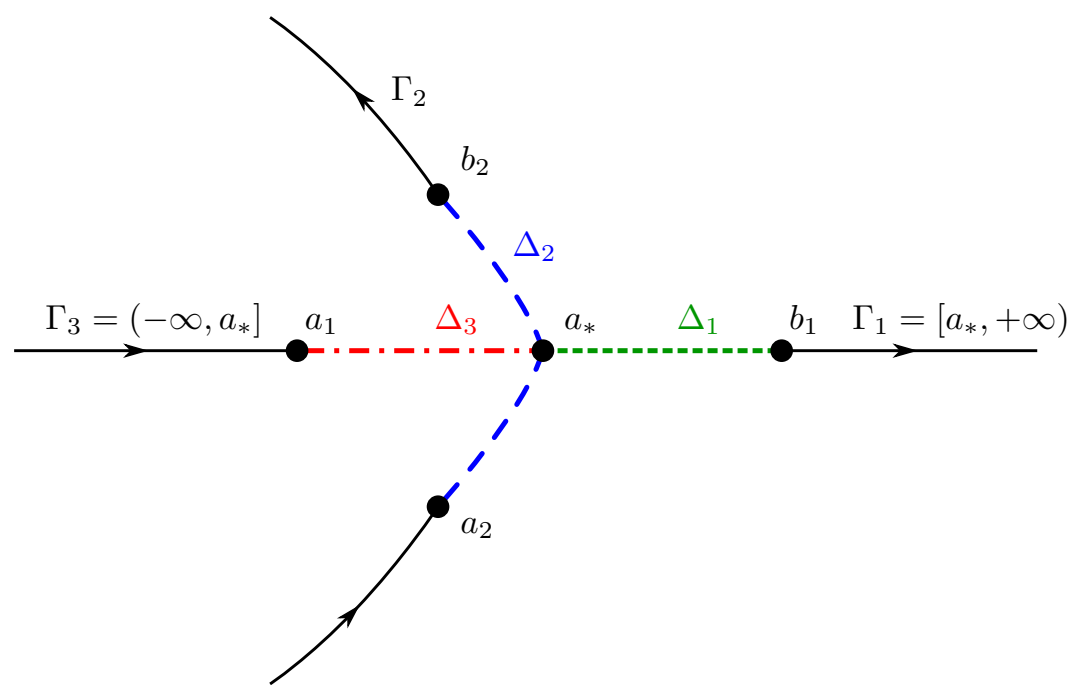

Figure 14. Pictorial representation of the sets $\Delta_{1}, \Delta_{2}, \Delta_{3}, \Gamma_{1}$, $\Gamma_{2}$ and $\Gamma_{3}$ in the supercritical regime.

- $\boldsymbol{Y}$ has continuous boundary values $\boldsymbol{Y}_{ \pm}$on $\Gamma_{Y}$, and $\boldsymbol{Y}_{+}(z)=\boldsymbol{Y}_{-}(z) \boldsymbol{J}_{Y}(z)$ for $z \in \Gamma_{Y}$, with ${ }^{1}$

$$
\boldsymbol{J}_{Y}(z) \stackrel{\text { def }}{=} \begin{cases}\boldsymbol{I}+e^{-N z^{3}} \boldsymbol{M}_{12}, & z \in \Gamma_{1} \\ \boldsymbol{I}+e^{-N z^{3}}\left(\boldsymbol{M}_{12}+\boldsymbol{M}_{13}\right), & z \in \Gamma_{2} \cap \mathbb{H}_{-}, \\ \boldsymbol{I}+e^{-N z^{3}} \boldsymbol{M}_{13}, & z \in \Gamma_{2} \cap \mathbb{H}_{+} .\end{cases}
$$

- $\boldsymbol{Y}(z)=\left(\boldsymbol{I}+\mathcal{O}\left(z^{-1}\right)\right) \operatorname{diag}\left(z^{N}, z^{-n}, z^{-m}\right), z \rightarrow \infty$.

- $\boldsymbol{Y}(z)$ is bounded as $z \rightarrow a_{*}$.

Standard arguments imply that $\operatorname{det} \boldsymbol{Y} \equiv 1$, so that $\boldsymbol{Y}$ is invertible everywhere on the plane. As shown in 21, the polynomial $P_{n, m}$ coincides with the $(1,1)$ entry of the matrix $\boldsymbol{Y}$, while polynomials $A_{n, m}$ and $B_{n, m}$ are (up to a factor $2 \pi i$ ) the $(2,1)$ and $(3,1)$ entries of the inverse matrix $\boldsymbol{Y}^{-1}$, respectively.

\section{Steepest Descent Analysis}

We now use the Riemann-Hilbert problem characterization, discussed in the previous section, as the starting point for our steepest descent analysis whose goal is to establish the detailed asymptotics of the solution $\boldsymbol{Y}$ above under the regime

$$
N=n+m \rightarrow \infty, \quad \frac{n}{N} \rightarrow \alpha \in(0,1 / 2) \backslash\left\{\alpha_{c}\right\}, \quad \frac{m}{N} \rightarrow 1-\alpha .
$$

For the moment we actually assume and additional constraint,

$$
\frac{n}{N}=\alpha, \quad \frac{m}{N}=1-\alpha,
$$

\footnotetext{
${ }^{1}$ Here and in what follows we will allow a slight abuse of notation for the sake of simplicity: whenever we speak about boundary values of a function on a set $\Gamma$, we refer to its values on $\stackrel{\circ}{\Gamma}$, where they are well defined, although frequently dropping the superscript $\circ$.
} 


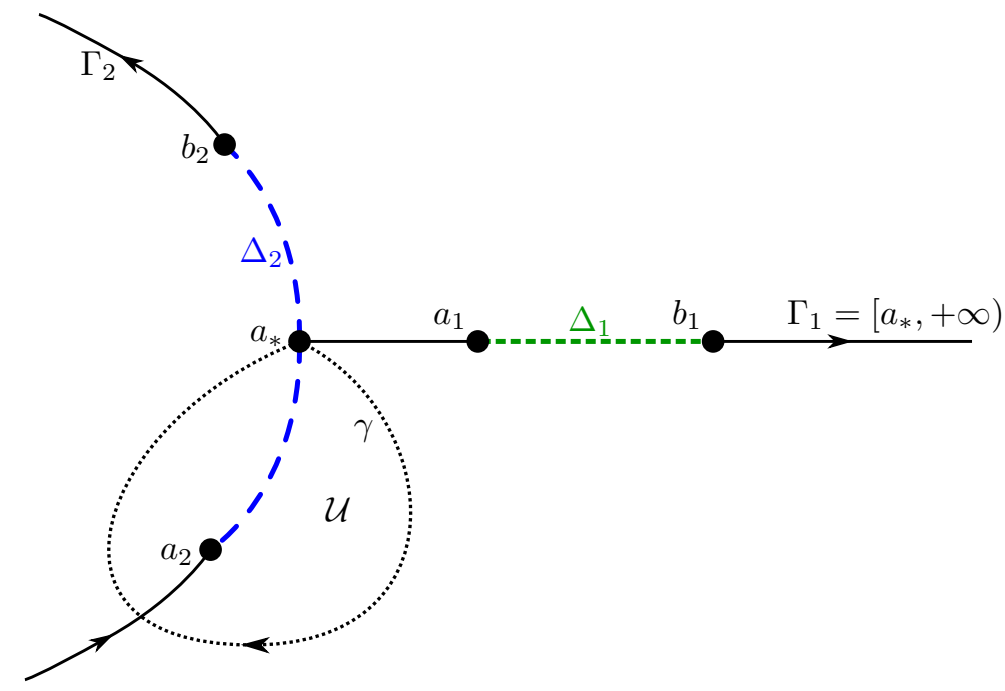

FiguRe 15. Pictorial representation of the contour $\Gamma_{X}=\Gamma_{1} \cup \Gamma_{2} \cup$ $\gamma$ and the region $\mathcal{U}$ in the subcritical regime.

so that, in particular, we are restricted to $\alpha \in \mathbb{Q}$. This restriction is only for convenience, and in Section 7 we extend our analysis to the general situation.

Recall that the Deift-Zhou nonlinear steepest descent method consists in a number of transformations of our Riemann-Hilbert characterization above in order to bring it to an equivalent "close-to-identity" problem. We will perform this analysis for the subcritical and supercritical cases in parallel.

6.1. Preliminary transformation. Our first goal is to modify slightly the structure of the jump matrix $\boldsymbol{J}_{Y}$ in (5.1) in a neighborhood of the curve $\Delta_{2}$ in the lower half plant $\AA^{2}$

To this end, we consider a closed Jordan contour $\gamma \subset \overline{\mathbb{H}}_{-}$, oriented clockwise, starting and ending at $a_{*}$ and encircling $\Delta_{2} \cap \mathbb{H}_{-}$. By Proposition 4.2, we can always take $\gamma \cap \mathbb{H}_{-}$lying in the domain $\Omega_{+}$, where $\operatorname{Re} \Phi_{3}(z)>0$, and choose $\gamma$ arbitrarily close to the set $E_{\alpha} \cap \mathbb{H}_{-}$, see Definition 4.3. We assume also that for some $\varepsilon>0$,

$$
\gamma \cap \mathbb{R}= \begin{cases}\left\{a_{*}\right\}, & \tau<\tau_{c}, \\ {\left[a_{1}-\varepsilon, a_{*}+\varepsilon\right],} & \tau>\tau_{c},\end{cases}
$$

see Figures 15 and 16 . We denote by $\mathcal{U} \supset\left(\Delta_{2} \cap \mathbb{H}_{-}\right)$the bounded domain in $\mathbb{H}_{-}$ encircled by $\gamma$.

We start with the transformation

$$
\boldsymbol{X}(z) \stackrel{\text { def }}{=} \begin{cases}\boldsymbol{Y}(z)\left(\boldsymbol{I}-\boldsymbol{M}_{32}\right), & z \in \mathcal{U}, \\ \boldsymbol{Y}(z), & \text { otherwise },\end{cases}
$$

so that $\boldsymbol{X}$ satisfies the following RHP:

$$
\text { - } \boldsymbol{X}: \mathbb{C} \backslash \Gamma_{X} \rightarrow \mathbb{C}^{3 \times 3} \text { is analytic, where } \Gamma_{X}=\Gamma_{Y} \cup \gamma \text {; }
$$

${ }^{2}$ At this stage, it might appear a technical step, but it will turn out to be linked to the essence of the problem. 


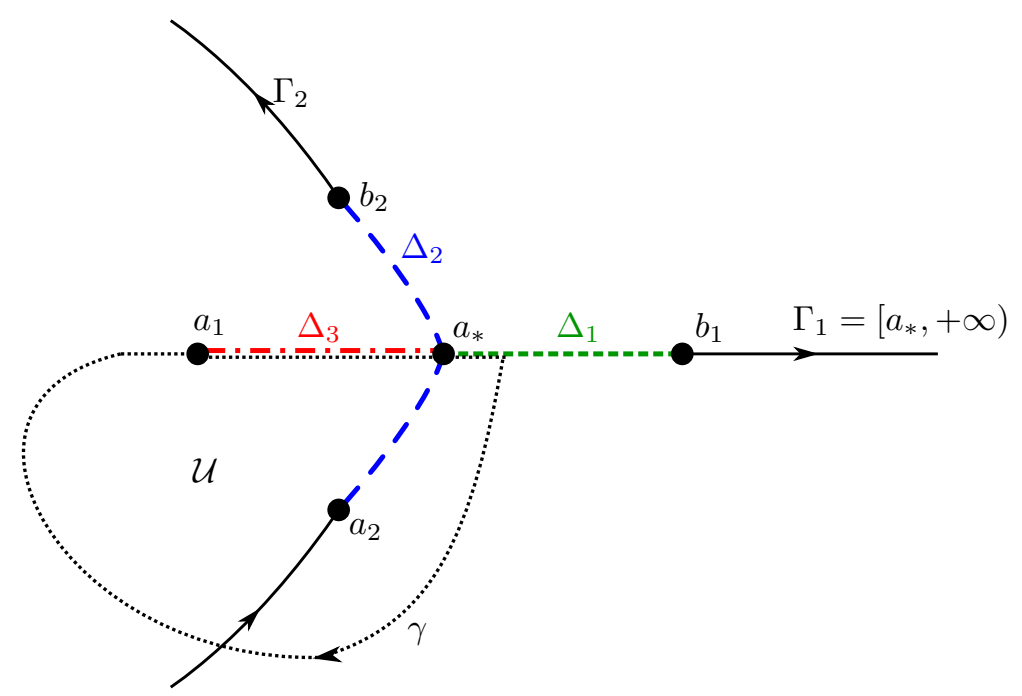

Figure 16. Pictorial representation of the contour $\Gamma_{X}=\Gamma_{1} \cup \Gamma_{2} \cup$ $\gamma$ and the region $\mathcal{U}$ in the supercritical regime.

- $\boldsymbol{X}$ has continuous boundary values $\boldsymbol{X}_{ \pm}$on $\Gamma_{X}$, and $\boldsymbol{X}_{+}(z)=\boldsymbol{X}_{-}(z) \boldsymbol{J}_{X}(z)$ for $z \in \Gamma_{X}$, with

$$
\boldsymbol{J}_{X}(z) \stackrel{\text { def }}{=} \begin{cases}\boldsymbol{I}+e^{-N z^{3}} \boldsymbol{M}_{12}, & z \in \Gamma_{1} \backslash \gamma \\ \boldsymbol{I}+e^{N z^{3}} \boldsymbol{M}_{12}+\boldsymbol{M}_{32}, & z \in \Delta_{1} \cap \gamma\left(\text { if } \tau>\tau_{c}\right), \\ \boldsymbol{I}+e^{-N z^{3}} \boldsymbol{M}_{13}, & z \in \Gamma_{2} \cap\left(\mathcal{U} \cup \mathbb{H}_{+}\right), \\ \boldsymbol{I}+e^{-N z^{3}}\left(\boldsymbol{M}_{12}+\boldsymbol{M}_{13}\right), & z \in\left(\Gamma_{2} \cap \mathbb{H}_{-}\right) \backslash \mathcal{U}, \\ \boldsymbol{I}+\boldsymbol{M}_{32}, & z \in \gamma \backslash \Delta_{1},\end{cases}
$$

- $\boldsymbol{X}(z)=\left(\boldsymbol{I}+\mathcal{O}\left(z^{-1}\right)\right) \operatorname{diag}\left(z^{N}, z^{-n}, z^{-m}\right), \quad$ as $z \rightarrow \infty$.

- $\boldsymbol{X}(z)$ is bounded at all boundary points of the jump contours.

6.2. First transformation. Now we normalize the behavior of the RHP at infinity, using functions $g_{j}$ 's and constants $r_{j}$ 's, defined in (3.19) and 3.22), respectively. Let $\Gamma_{T}=\Gamma_{X}$, and set

$$
\begin{aligned}
\boldsymbol{T}(z) \stackrel{\text { def }}{=} & \operatorname{diag}\left(e^{-N r_{1}}, e^{-N r_{2}}, e^{-N r_{3}}\right) \boldsymbol{X}(z) \\
& \times \operatorname{diag}\left(e^{N\left(g_{1}(z)-\frac{2}{3} z^{3}\right)}, e^{N\left(g_{2}(z)+\frac{1}{3} z^{3}\right)}, e^{N\left(g_{3}(z)+\frac{1}{3} z^{3}\right)}\right), \quad z \in \mathbb{C} \backslash \Gamma_{T} .
\end{aligned}
$$

A priori, $\boldsymbol{T}$ is analytic on $\mathbb{C} \backslash\left(\Gamma_{T} \cup\left(-\infty, a_{*}\right]\right)$, and by the assumption 6.1,

$$
\boldsymbol{T}(z)=\boldsymbol{I}+\mathcal{O}\left(z^{-1}\right), \quad \text { as } z \rightarrow \infty,
$$

which was the primary goal of this transformation.

We analyze the jumps of $\boldsymbol{T}$ on the curves comprising $\Gamma_{T} \cup\left(-\infty, a_{*}\right)$; it is convenient to use here the notation

$$
\operatorname{diag} e^{N \Delta g}=\operatorname{diag}\left(e^{N \Delta g_{1}}, e^{N \Delta g_{2}}, e^{N \Delta g_{3}}\right), \quad \Delta g_{j}=g_{j+}-g_{j-} .
$$


Using the value of the periods $(3.17)$, we see that on $\mathbb{R} \backslash \Gamma_{1}=\left(-\infty, a_{*}\right)$,

$$
g_{1+}-g_{1-}=\oint_{\Delta_{1} \cup \Delta_{2}} \xi_{1}=-2 \pi i .
$$

In the same vein, on $\mathbb{R} \backslash\left(\Gamma_{1} \cup \Delta_{3}\right)$,

$$
\begin{aligned}
& g_{2+}-g_{2-}=\oint_{\Delta_{1} \cup \Delta_{3}} \xi_{2}=2 \pi i \alpha, \\
& g_{3+}-g_{3-}=\oint_{\Delta_{2} \cup \Delta_{3}} \xi_{3}=2 \pi i(1-\alpha),
\end{aligned}
$$

and again, due to the crucial assumption (6.1), in 6.5 - 6.6), all expressions of the form $N \Delta g_{i}$ are integer multiples of $2 \pi i$. In particular, this shows that $\boldsymbol{T}$ is actually analytic on $\mathbb{C} \backslash \Gamma_{T}$. From the RHP for $\boldsymbol{X}$ we get that $\boldsymbol{T}_{+}(z)=\boldsymbol{T}_{-}(z) \boldsymbol{J}_{T}(z), z \in \Gamma_{T}$, with

$$
\begin{aligned}
& \boldsymbol{J}_{T}= \\
& \begin{cases}\operatorname{diag} e^{N \Delta g}+e^{N\left(g_{2+}-g_{1-}\right)} M_{12}, & z \in \Gamma_{1} \backslash \gamma, \\
\operatorname{diag} e^{N \Delta g}+e^{N\left(g_{2+}-g_{1-}\right)} M_{12}+e^{N\left(g_{2+}-g_{3-}\right)} M_{32}, & z \in \gamma \cap \Delta_{1}, \quad\left(\text { if } \tau>\tau_{c}\right), \\
\operatorname{diag} e^{N \Delta g}+e^{N\left(g_{3+}-g_{1-}\right)} M_{13}, & z \in \Gamma_{2} \cap\left(\mathcal{U} \cup \mathbb{H}_{+}\right), \\
\operatorname{diag} e^{N \Delta g}+e^{N\left(g_{2+}-g_{1-}\right)} M_{12}+e^{N\left(g_{3+}-g_{1-}\right)} M_{13}, & z \in\left(\Gamma_{2} \cap \mathbb{H}_{-}\right) \backslash \mathcal{U}, \\
\operatorname{diag} e^{N \Delta g}+e^{N\left(g_{2+}-g_{3-}\right)} M_{32}, & z \in \gamma \backslash \Delta_{1},\end{cases}
\end{aligned}
$$

where we have used the structure of cuts in the definition in (3.19).

The expression for some entries of the jump matrix $\boldsymbol{J}_{T}$ can be simplified, at least on subsets of the jump contours:

(i) On $\Gamma_{1}$, by 3.20 ,

$$
g_{2+}(z)-g_{1-}(z)=\int_{b_{1}}^{z}\left(\xi_{2+}-\xi_{1-}\right) d s+c_{2}-c_{1}=\int_{b_{1}}^{z}\left(\xi_{2+}-\xi_{1-}\right) d s .
$$

Since $\xi_{2+}=\xi_{1-}$ on $\stackrel{\circ}{\Delta}_{1}:=\Delta_{1} \backslash\left\{\max \left(a_{1}, a_{*}\right), b_{1}\right\}$ (see (3.9)), we conclude in particular that $g_{2+}(z)-g_{1-}(z)=0, z \in \Delta_{1}$.

(ii) On $\Delta_{2} \cap \mathbb{H}_{-}$, by (3.10) and (3.20),

$$
\begin{aligned}
g_{3+}(z)-g_{1-}(z) & =\int_{a_{2}}^{z}\left(\xi_{3+}-\xi_{1-}\right) d s+\int_{a_{*}}^{a_{2}}\left(\xi_{3-}-\xi_{1-}\right) d s \\
& -\int_{b_{1}}^{a_{*}} \xi_{1-} d s+c_{3}-c_{1}=\int_{a_{2}}^{z}\left(\xi_{3+}-\xi_{1-}\right) d s=0 .
\end{aligned}
$$

On the other hand, by 3.21 we have on $\Delta_{2} \cap \mathbb{H}_{+}$:

$$
g_{3+}(z)-g_{1-}(z)=c_{3}-\overline{c_{3}}-c_{1}+\overline{c_{1}} .
$$

Using (3.17) and (3.20) we get that

$$
g_{3+}(z)-g_{1-}(z)=\oint_{\Delta_{1} \cup \Delta_{2}} \xi_{1}=2 \pi i \quad \text { on } \Delta_{2} \cap \mathbb{H}_{+} .
$$

(iii) On $\Delta_{3}$ (for $\tau>\tau_{c}$ ), by $(3.9),(3.12)$ and 3.20 ,

$$
g_{2+}(z)-g_{3-}(z)=\int_{a_{*}}^{z}\left(\xi_{2+}-\xi_{3-}\right)+\int_{b_{1}}^{a_{*}}\left(\xi_{2+}-\xi_{1-}\right)=0,
$$


and analogously,

$$
g_{3+}(z)-g_{2-}(z)=\oint_{\Delta_{1} \cup \Delta_{2}} \xi_{1}=2 \pi i
$$

(iv) On $\left(a_{*}, a_{1}\right)$, for $\tau<\tau_{c}$,

$$
\begin{aligned}
& g_{1+}-g_{1-}=\oint_{\Delta_{1}} \xi_{1}=\oint_{\Delta_{1}} \xi_{2}=-2 \pi i \alpha \\
& g_{2+}-g_{2-}=\oint_{\Delta_{1}} \xi_{2}=2 \pi i \alpha .
\end{aligned}
$$

Gathering these identities, we conclude that $\boldsymbol{T}$ satisfies the following RHP (see Figures 15 and 16):

- $\boldsymbol{T}: \mathbb{C} \backslash \Gamma_{T} \rightarrow \mathbb{C}^{3 \times 3}$ is analytic;

- $\boldsymbol{T}$ has continuous boundary values $\boldsymbol{T}_{ \pm}$on $\Gamma_{T}$, and $\boldsymbol{T}_{+}(z)=\boldsymbol{T}_{-}(z) \boldsymbol{J}_{T}(z)$, $z \in \Gamma_{T}$, with

$$
\boldsymbol{J}_{T}= \begin{cases}\operatorname{diag}\left(e^{N\left(g_{1+}-g_{1-}\right)}, e^{N\left(g_{2+}-g_{2}\right)}, 1\right)+\boldsymbol{M}_{12}, & z \in \Delta_{1} \backslash \gamma, \\ \operatorname{diag}\left(e^{N\left(g_{1+}-g_{1-}\right)}, e^{N\left(g_{2+}-g_{2}-\right)}, 1\right) & \\ \quad+\boldsymbol{M}_{12}+e^{N\left(g_{2+}-g_{3-}\right)} \boldsymbol{M}_{32}, & z \in \Delta_{1} \cap \gamma\left(\text { if } \tau>\tau_{c}\right), \\ \boldsymbol{I}+e^{N\left(g_{2+}-g_{1-}\right)} \boldsymbol{M}_{12}, & z \in \Gamma_{1} \backslash \Delta_{1}, \\ \operatorname{diag}\left(e^{N\left(g_{1+}-g_{1-}\right)}, 1, e^{N\left(g_{3+}-g_{3}\right)}\right)+\boldsymbol{M}_{13}, & z \in \Delta_{2}, \\ \boldsymbol{I}+e^{N\left(g_{3}-g_{1}\right)} \boldsymbol{M}_{13}, & z \in \Gamma_{2} \cap\left(\mathcal{U} \cup \mathbb{H}_{+}\right) \backslash \Delta_{2}, \\ \boldsymbol{I}+e^{N\left(g_{2}-g_{1}\right)} \boldsymbol{M}_{12}+e^{N\left(g_{3}-g_{1}\right)} \boldsymbol{M}_{13}, & z \in\left(\Gamma_{2} \cap \mathbb{H}-\right) \backslash \mathcal{U}, \\ \operatorname{diag}\left(1, e^{N\left(g_{2+}-g_{2-}\right)}, e^{N\left(g_{3+}-g_{3-}\right)}\right)+\boldsymbol{M}_{32}, & z \in \Delta_{3}\left(\text { if } \tau>\tau_{c}\right), \\ \boldsymbol{I}+e^{N\left(g_{2}-g_{3}\right)} \boldsymbol{M}_{32}, & z \in \gamma \backslash \mathbb{R} .\end{cases}
$$

- $\boldsymbol{T}(z)=\boldsymbol{I}+\mathcal{O}\left(z^{-1}\right)$ as $z \rightarrow \infty$.

- $\boldsymbol{T}(z)$ is bounded at all end points of the analytic arcs comprising $\Gamma_{T}$.

Remark 6.1. Notice that the jump on $\left(\gamma \cap \Gamma_{3}\right) \backslash \Delta_{3}$ has the form $\boldsymbol{I}+e^{N\left(g_{2+}-g_{3-}\right)} \boldsymbol{M}_{32}$. However, due to (6.6) we can rewrite it as $\boldsymbol{I}+e^{N\left(g_{2-}-g_{3-}\right)} \boldsymbol{M}_{32}$, which justifies stating that $\boldsymbol{J}_{T}=\boldsymbol{I}+e^{N\left(g_{2}-g_{3}\right)} \boldsymbol{M}_{32}$ on $\gamma \backslash\left(\Delta_{1} \cup \Delta_{3}\right)$, understanding that on the portion of $\gamma$ along $\mathbb{R}$ we use the "-" boundary values of the entries.

An important fact about $\boldsymbol{J}_{T}$ is that not all its components are asymptotically relevant, since some of the off-diagonal entries of $\boldsymbol{J}_{T}$ exhibit an exponential decay. Indeed,

- On $\Gamma_{1} \backslash \Delta_{1}$, as we have seen,

$$
g_{2+}-g_{1-}=\int_{b_{1}}^{z}\left(\xi_{2+}-\xi_{1-}\right) d s \begin{cases}<0, & z>b_{1}, \\ <0, & a_{*}<z<a_{1}\left(\text { when } \tau<\tau_{c}\right),\end{cases}
$$

where the inequalities are a direct consequence of $(3.3)-(3.6)$.

- On $\gamma \backslash \mathbb{R}$, by (3.28),

$$
g_{2}(z)-g_{3}(z)=-\Phi_{3}(z)-2 \pi i \alpha+ \begin{cases}\int_{a_{*}}^{a_{1}}\left(\xi_{1}-\xi_{2}\right) d s+c_{2}, & 0<\tau<\tau_{c}, \\ 0, & \tau_{c}<\tau<1 / 4\end{cases}
$$


with $\Phi_{3}$ defined in 3.25 . It remains to use that $c_{2} \in i \mathbb{R}$, Proposition 4.2 , and the fact that by $(3.3), \xi_{1}<\xi_{2}<\xi_{3}$ on $\left[a_{*}, a_{1}\right)$ for $0<\tau<\tau_{c}$, in order to conclude that $\operatorname{Re}\left(g_{2}-g_{3}\right)<0$ on $\gamma \backslash \mathbb{R}$ (but uniformly up to $a_{*}$ for $\tau<\tau_{c}$ ).

- Finally, by (3.26), (3.27) and Proposition 4.2, we can always take $\Gamma_{2}$ in such a way that on $\Gamma_{2} \cap \mathbb{H}_{-} \backslash \Delta_{2}$,

$$
\begin{aligned}
& \operatorname{Re}\left(g_{2}-g_{1}\right)(z)=-\operatorname{Re} \Phi_{1}(z)<0, \\
& \operatorname{Re}\left(g_{3}-g_{1}\right)(z)=-\operatorname{Re} \Phi_{2}(z)<0 .
\end{aligned}
$$

In summary, on some contours of $\Gamma_{T}$, the jump matrix $\boldsymbol{J}_{T}$ is exponentially close to $\boldsymbol{I}$, while on others, it has entries with oscillatory behavior. The (now) standard technique to deal with these oscillations is to split the jump contour there in several new contours using the classical factorization

$$
\left(\begin{array}{cc}
e^{-A} & 1 \\
0 & e^{A}
\end{array}\right)=\left(\begin{array}{cc}
1 & 0 \\
e^{A} & 1
\end{array}\right)\left(\begin{array}{cc}
0 & 1 \\
-1 & 0
\end{array}\right)\left(\begin{array}{cc}
1 & 0 \\
e^{-A} & 1
\end{array}\right)=\boldsymbol{L C} \boldsymbol{R}
$$

with the obvious choices for the notation $\boldsymbol{L}, \boldsymbol{C}, \boldsymbol{R}$. This is the next step, in which it is convenient to treat the subcritical and supercritical cases separately.

Motivated by (6.14), we will make use of a special notation for the following $3 \times 3$ matrices:

$$
\sigma_{3 j} \stackrel{\text { def }}{=} \begin{cases}\boldsymbol{M}_{12}-\boldsymbol{M}_{21}+\boldsymbol{M}_{33}, & \text { if } j=1, \\ \boldsymbol{M}_{13}-\boldsymbol{M}_{31}+\boldsymbol{M}_{22}, & \text { if } j=2, \\ \boldsymbol{M}_{32}-\boldsymbol{M}_{23}+\boldsymbol{M}_{11}, & \text { if } j=3 .\end{cases}
$$

For instance,

$$
\boldsymbol{\sigma}_{31}=\left(\begin{array}{ccc}
0 & 1 & 0 \\
-1 & 0 & 0 \\
0 & 0 & 1
\end{array}\right)
$$

etcetera. A feature of these matrices, useful in what follows, is that $\boldsymbol{\sigma}_{3 j}^{T}=\boldsymbol{\sigma}_{3 j}^{-1}$, $j=1,2,3$.

6.3. Opening of lenses in the precritical case. Let us start with the case $0<\tau<\tau_{c}$, when 6.12$)$ simplifies to

$$
J_{T}= \begin{cases}\operatorname{diag}\left(e^{N\left(g_{1+}-g_{1-}\right)}, e^{N\left(g_{2+}-g_{2-}\right)}, 1\right)+\boldsymbol{M}_{12}, & z \in \Delta_{1}, \\ \operatorname{diag}\left(e^{N\left(g_{1+}-g_{1-}\right)}, 1, e^{N\left(g_{3+}-g_{3}\right)}\right)+\boldsymbol{M}_{13}, & z \in \Delta_{2}, \\ \boldsymbol{I}+e^{N\left(g_{2+}-g_{1-}\right)} \boldsymbol{M}_{12}, & z \in \Gamma_{1} \backslash \Delta_{1}, \\ \boldsymbol{I}+e^{N\left(g_{3}-g_{1}\right)} \boldsymbol{M}_{13}, & z \in \Gamma_{2} \cap\left(\mathcal{U} \cup \mathbb{H}_{+}\right) \backslash \Delta_{2}, \\ \boldsymbol{I}+e^{N\left(g_{2}-g_{1}\right)} \boldsymbol{M}_{12}+e^{N\left(g_{3}-g_{1}\right)} \boldsymbol{M}_{13}, & z \in\left(\Gamma_{2} \cap \mathbb{H}_{-}\right) \backslash \mathcal{U}, \\ \boldsymbol{I}+e^{N\left(g_{2}-g_{3}\right)} \boldsymbol{M}_{32}, & z \in \gamma .\end{cases}
$$

It is convenient to rewrite the jumps on $\Delta_{1}$ and $\Delta_{2}$ in terms of the functions $\Phi_{j}$ introduced in (3.23)-3.25). First, by (3.9), $g_{1 \pm}(z)=g_{2 \mp}(z)$ for $z \in \Delta_{1}$, and this gives us the identities below:

$$
\begin{aligned}
g_{1+}(z)-g_{1-}(z) & =\Phi_{1+}(z), & & z \in \Delta_{1}, \\
g_{2+}(z)-g_{2-}(z) & =\Phi_{1-}(z), & & z \in \Delta_{1}, \\
\Phi_{1+}(z)+\Phi_{1-}(z) & =0, & & z \in \Delta_{1} .
\end{aligned}
$$




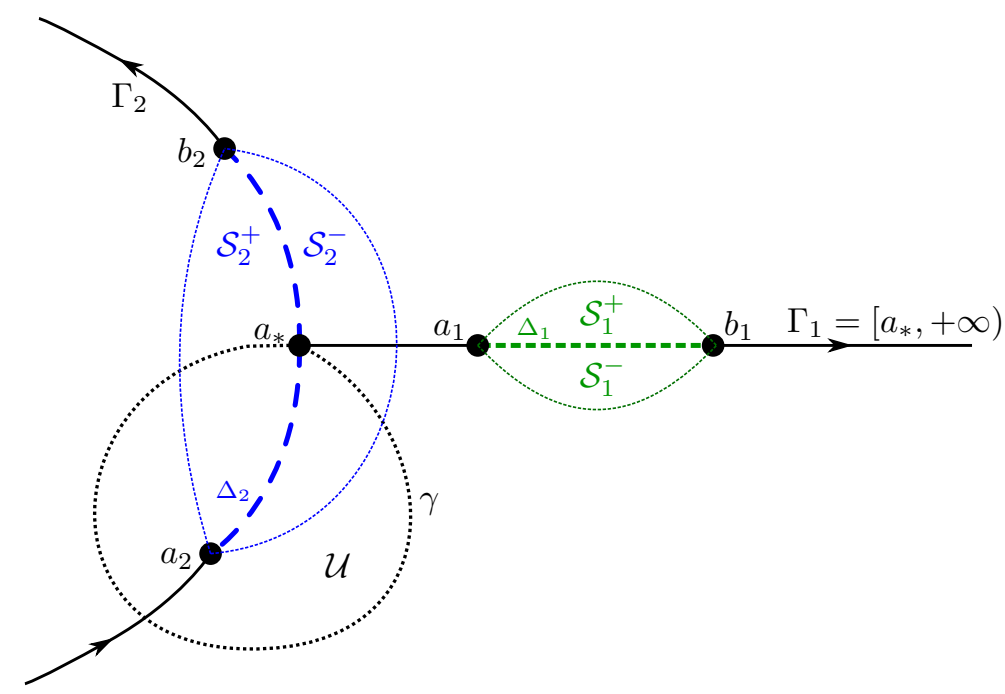

Figure 17. Pictorial representation of the contour $\Gamma_{S}=\Gamma_{X} \cup$ $\partial \mathcal{S}_{1} \cup \partial \mathcal{S}_{2}$ in the subcritical regime.

In consequence, using (6.14) and 6.16), we can rewrite the jump on $\Delta_{1}$ as

$$
\begin{aligned}
\boldsymbol{J}_{T} & =\left(\begin{array}{ccc}
e^{N \Phi_{1+}} & 1 & 0 \\
0 & e^{N \Phi_{1-}} & 0 \\
0 & 0 & 1
\end{array}\right) \\
& =\left(\begin{array}{ccc}
1 & 0 & 0 \\
e^{N \Phi_{1-}} & 1 & 0 \\
0 & 0 & 1
\end{array}\right)\left(\begin{array}{ccc}
0 & 1 & 0 \\
-1 & 0 & 0 \\
0 & 0 & 1
\end{array}\right)\left(\begin{array}{ccc}
1 & 0 & 0 \\
e^{N \Phi_{1+}} & 1 & 0 \\
0 & 0 & 1
\end{array}\right) \\
& =\left(\boldsymbol{I}+e^{N \Phi_{1-}} \boldsymbol{M}_{21}\right) \boldsymbol{\sigma}_{31}\left(\boldsymbol{I}+e^{N \Phi_{1+}} \boldsymbol{M}_{21}\right),
\end{aligned}
$$

with $\boldsymbol{\sigma}_{31}$ defined in 6.15$)$. In the same vein, by $(3.10),(6.7)$ and 6.8$)$,

$$
\begin{aligned}
g_{1+}(z)-g_{1-}(z) & \equiv \Phi_{2+}(z) \quad \bmod (2 \pi i), & z \in \Delta_{2}, \\
g_{3+}(z)-g_{3-}(z) & \equiv \Phi_{2-}(z) \quad \bmod (2 \pi i), & z \in \Delta_{2}, \\
\Phi_{2+}(z)+\Phi_{2-}(z) \equiv 0 \quad \bmod (2 \pi i), & & z \in \Delta_{2},
\end{aligned}
$$

and again (6.14) and now (6.17) yield that $J_{T}$ on $\Delta_{2}$ has the form

$$
\boldsymbol{J}_{T}=\left(\boldsymbol{I}+e^{N \Phi_{2-}} \boldsymbol{M}_{31}\right) \boldsymbol{\sigma}_{32}\left(\boldsymbol{I}+e^{N \Phi_{2+}} \boldsymbol{M}_{31}\right),
$$

where $\boldsymbol{\sigma}_{32}$ was defined in 6.15.

These factorizations of the jumps across $\Delta_{1}$ and $\Delta_{2}$ motivate to introduce new domains (or "to open lenses") $\mathcal{S}_{j}=\mathcal{S}_{j}^{+} \cup \mathcal{S}_{j}^{-}$around $\Delta_{j}, j=1,2$ (see Figure 17), and define

$$
\boldsymbol{S}(z) \stackrel{\text { def }}{=} \boldsymbol{T}(z) \times \begin{cases}\boldsymbol{I}, & z \in \mathbb{C} \backslash \mathcal{S}_{1} \cup \mathcal{S}_{2}, \\ \boldsymbol{I} \mp e^{N \Phi_{1}} \boldsymbol{M}_{21}, & z \in \mathcal{S}_{1}^{ \pm} \\ \boldsymbol{I} \mp e^{N \Phi_{2}} \boldsymbol{M}_{31}, & z \in \mathcal{S}_{2}^{ \pm} .\end{cases}
$$

Observe that on $\left(a_{*}, a_{1}\right)$ by 6.11,

$$
\Phi_{2+}(z)-\Phi_{2-}(z)=g_{1+}(z)-g_{1-}(z)=-2 \pi i \alpha, \quad z \in\left(a_{*}, a_{1}\right),
$$


so that, in particular, $e^{N \Phi_{2+}(z)}=e^{N \Phi_{2-}(z)}$ on $\left(a_{*}, a_{1}\right)$. Moreover, as a consequence of 3.26 and 6.11),

$$
g_{2+}(z)-g_{1-}(z)=-\Phi_{1+}(z)-2 \pi i \alpha, \quad z \in\left(a_{*}, a_{1}\right) .
$$

Hence, $\boldsymbol{S}$ satisfies the following RHP (see Figure 17):

- $\boldsymbol{S}: \mathbb{C} \backslash \Gamma_{S} \rightarrow \mathbb{C}^{3 \times 3}$ is analytic, where $\Gamma_{S}=\Gamma_{T} \cup \partial \mathcal{S}_{1} \cup \partial \mathcal{S}_{2}$;

- $\boldsymbol{S}$ has continuous boundary values $\boldsymbol{S}_{ \pm}$on $\Gamma_{S}$, and $\boldsymbol{S}_{+}(z)=\boldsymbol{S}_{-}(z) \boldsymbol{J}_{S}(z), z \in$ $\Gamma_{S}$, with

$$
\boldsymbol{J}_{S}= \begin{cases}\boldsymbol{I}+e^{N \Phi_{j}} \boldsymbol{M}_{j+1,1}, & z \in \partial \mathcal{S}_{j}^{ \pm}, \quad j=1,2, \\ \boldsymbol{\sigma}_{3, j}, & z \in \Delta_{j}, \quad j=1,2, \\ \boldsymbol{I}+e^{-N \Phi_{1+}}\left(\boldsymbol{M}_{12}-e^{N \Phi_{2-}} \boldsymbol{M}_{32}\right), & z \in\left(a_{*}, a_{1}\right) \cap \mathcal{S}_{2}, \\ \boldsymbol{J}_{T}, & \text { elsewhere on } \Gamma_{S} .\end{cases}
$$

- $\boldsymbol{S}(z)=I+\mathcal{O}\left(z^{-1}\right)$ as $z \rightarrow \infty$.

- $\boldsymbol{S}(z)$ is bounded at all end points of the analytic arcs comprising $\Gamma_{S}$.

Observe that the jumps on $\Delta_{1}$ and $\Delta_{2}$ now are independent of $N$.

We claim that we can select the domains $\mathcal{S}_{j}$ in such a way that the rest of the jumps are exponentially close to $\boldsymbol{I}$. Indeed, by (3.3), $\operatorname{Re} \Phi_{1+}(x)>0$ for $x \in\left[a_{*}, a_{1}\right)$. Furthermore, recall that $\mu_{j}$ from (3.18) are positive measures, and we can conclude that

$$
\begin{array}{ll}
-d \Phi_{1+}(s)=\left(\xi_{1+}(s)-\xi_{2+}(s)\right) d s \in i \mathbb{R}_{+}, & s \in \Delta_{1}, \\
-d \Phi_{2+}(s)=\left(\xi_{1+}(s)-\xi_{3+}(s)\right) d s \in i \mathbb{R}_{+}, & s \in \Delta_{2} .
\end{array}
$$

Thus,

$$
\Phi_{j \pm}(z) \in i \mathbb{R}_{\mp}, \quad z \in \Delta_{j}, \quad j=1,2 .
$$

This is enough to assure that the domains $\mathcal{S}_{j}$ can be taken such that

$$
\operatorname{Re} \Phi_{j}(z)<0, \quad z \in \overline{\mathcal{S}_{j}^{ \pm}} \backslash \Delta_{j}, \quad j=1,2 ;
$$

we assume that in what follows the inequalities $\sqrt{6.22}$ hold.

An important consequence of $(6.13), \sqrt{6.16}),(6.21)$, and $(6.22)$, as well as of our analysis at the end of Section 6.2 is that away from $\Delta_{1}$ and $\Delta_{2}$, the jump matrix $\boldsymbol{J}_{S}$ is exponentially close to $\boldsymbol{I}$. Moreover, this is true uniformly all the way up to $\Delta_{j}$ as long as we stay away from the end points $a_{j}, b_{j}$.

6.4. Opening of lenses in the supercritical case. By $\sqrt{6.2}$ and $\sqrt{6.12}$, for $\tau_{c}<\tau<1 / 4$,

$$
\boldsymbol{J}_{T}= \begin{cases}\operatorname{diag}\left(e^{N\left(g_{1+}-g_{1-}\right)}, e^{N\left(g_{2+}-g_{2-}\right)}, 1\right)+\boldsymbol{M}_{12}, & z \in \Delta_{1} \backslash \gamma=\left(a_{*}+\varepsilon, b_{1}\right), \\ \operatorname{diag}\left(e^{N\left(g_{1+}-g_{1-}\right)}, e^{N\left(g_{2+}-g_{2-}\right)}, 1\right) & \\ \quad+\boldsymbol{M}_{12}+e^{N\left(g_{2+}-g_{3-}\right)} \boldsymbol{M}_{32}, & z \in \Delta_{1} \cap \gamma=\left(a_{*}, a_{*}+\varepsilon\right), \\ \boldsymbol{I}+e^{N\left(g_{2}-g_{1}\right)} \boldsymbol{M}_{12}, & z \in \Gamma_{1} \backslash \Delta_{1}=\left(b_{1},+\infty\right), \\ \operatorname{diag}\left(e^{N\left(g_{1+}-g_{1-}\right)}, 1, e^{N\left(g_{3+}-g_{3}\right)}\right)+\boldsymbol{M}_{13}, & z \in \Delta_{2}, \\ \boldsymbol{I}+e^{N\left(g_{3}-g_{1}\right)} \boldsymbol{M}_{13}, & z \in \Gamma_{2} \cap\left(\mathcal{U} \cup \mathbb{H}_{+}\right) \backslash \Delta_{2}, \\ \boldsymbol{I}+e^{N\left(g_{2}-g_{1}\right)} \boldsymbol{M}_{12}+e^{N\left(g_{3}-g_{1}\right)} \boldsymbol{M}_{13}, & z \in\left(\Gamma_{2} \cap \mathbb{H}-\right) \backslash \mathcal{U}, \\ \operatorname{diag}\left(1, e^{N\left(g_{2+}-g_{2-}\right)}, e^{N\left(g_{3+}-g_{3-}\right)}\right)+\boldsymbol{M}_{32}, & z \in \Delta_{3}, \\ \boldsymbol{I}+e^{N\left(g_{2}-g_{3}\right)} \boldsymbol{M}_{32}, & z \in \gamma \backslash\left(\Delta_{1} \cup \Delta_{3}\right) .\end{cases}
$$


Again, we rewrite these jumps in terms of the functions $\Phi_{j}$, introduced in 3.23 6.25). On $\Delta_{3}$, by 6.5, 6.9 and 6.10 we now have

$$
\begin{aligned}
g_{2+}(z)-g_{2-}(z) & =\Phi_{3-}(z)+2 \pi i \alpha, & & z \in \Delta_{3}, \\
g_{3+}(z)-g_{3-}(z) & =\Phi_{3+}(z)+2 \pi i \alpha, & & z \in \Delta_{3}, \\
\Phi_{3+}(z)+\Phi_{3-}(z) & =2 \pi i(1-2 \alpha), & & z \in \Delta_{3} .
\end{aligned}
$$

As for $\Delta_{1}$ and $\Delta_{2}$, along with the relations $(6.16)$ and $(6.17)$, we get (using now (3.9) and (3.27),

$$
g_{2+}(z)-g_{3-}(z)=\Phi_{2-}(z)-2 \pi i, \quad z \in \Delta_{1}
$$

and

$$
\begin{aligned}
g_{1+}(z)-g_{1-}(z) & =\Phi_{2+}(z), & & z \in \Delta_{2}, \\
g_{3+}(z)-g_{3-}(z) & =\Phi_{2-}(z), & & z \in \Delta_{2}, \\
\Phi_{2+}(z)+\Phi_{2-}(z) & =0, & & z \in \Delta_{2}, \\
\Phi_{3+}(z)-\Phi_{1-}(z) & =2 \pi i(3 \alpha-1), & & z \in \Delta_{2} \cap \mathbb{H}_{+}, \\
\Phi_{3+}(z)-\Phi_{1-}(z) & =2 \pi i \alpha, & & z \in \Delta_{2} \cap \mathbb{H}_{-} ;
\end{aligned}
$$

furthermore,

$$
\begin{array}{ll}
\Phi_{2+}(z)-\Phi_{2-}(z)=-2 \pi i(2-\alpha), & z \in \gamma \cap \mathbb{R}, \\
\Phi_{2+}(z)-\Phi_{2-}(z)=-2 \pi i \alpha, & z \in\left(a_{*}, a_{1}\right) .
\end{array}
$$

All these identities are simple consequences of $3.26-(3.28)$ and of the periods (3.17).

Like in Section 6.3 , the identities above imply that

$$
\boldsymbol{J}_{T}= \begin{cases}\left(\boldsymbol{I}+e^{N \Phi_{1-}} \boldsymbol{M}_{21}\right) \boldsymbol{\sigma}_{31}\left(\boldsymbol{I}+e^{N \Phi_{1+}} \boldsymbol{M}_{21}\right), & z \in \Delta_{1} \backslash \gamma, \\ \left(\boldsymbol{I}+e^{N \Phi_{1-}} \boldsymbol{M}_{21}\right) \boldsymbol{\sigma}_{31}\left(\boldsymbol{I}+e^{N \Phi_{1+}} \boldsymbol{M}_{21}\right)\left(I+e^{N \Phi_{2-}} \boldsymbol{M}_{32}\right), & z \in \Delta_{1} \cap \gamma, \\ \left(\boldsymbol{I}+e^{N \Phi_{2-}} \boldsymbol{M}_{31}\right) \boldsymbol{\sigma}_{32}\left(\boldsymbol{I}+e^{N \Phi_{2+}} \boldsymbol{M}_{31}\right), & z \in \Delta_{2}, \\ \left(\boldsymbol{I}+e^{N \Phi_{3-}} \boldsymbol{M}_{23}\right) \boldsymbol{\sigma}_{33}\left(\boldsymbol{I}+e^{N \Phi_{3+}} \boldsymbol{M}_{23}\right), & z \in \Delta_{3},\end{cases}
$$

where $\boldsymbol{\sigma}_{33}$ was defined in 6.15). Moreover, from the fact that the measures in (3.18) are positive we conclude that

$$
\begin{aligned}
& \left(\xi_{1+}(s)-\xi_{2+}(s)\right) d s \in i \mathbb{R}_{+}, \quad s \in \Delta_{1}, \\
& \left(\xi_{1+}(s)-\xi_{3+}(s)\right) d s \in i \mathbb{R}_{+}, \quad s \in \Delta_{2}, \\
& \left(\xi_{3+}(s)-\xi_{2+}(s)\right) d s \in i \mathbb{R}_{+}, \quad s \in \Delta_{3} .
\end{aligned}
$$

As a consequence,

$$
\Phi_{j \pm}(z) \in i \mathbb{R}_{\mp}, \quad z \in \Delta_{j}, \quad j=1,2,3,
$$

which shows again that the inequalities

$$
\operatorname{Re} \Phi_{j}(z)<0
$$

hold true in a neighborhood of int $\Delta_{j}, j=1,2,3$.

These considerations motivate to open the lens $\mathcal{S}_{j}=\mathcal{S}_{j}^{+} \cup \mathcal{S}_{j}^{-}$around $\Delta_{j}$ so that 6.27) holds true on $\partial \mathcal{S}_{j}^{ \pm} \backslash\left\{a_{1}, a_{2}, b_{1}, b_{2}\right\}$. The geometry of the lens is shown in Figure 18. We can impose an additional requirement,

$$
\partial \mathcal{S}_{2}^{-} \cap S_{1}^{-}=\gamma \cap S_{1}^{-} \text {. }
$$




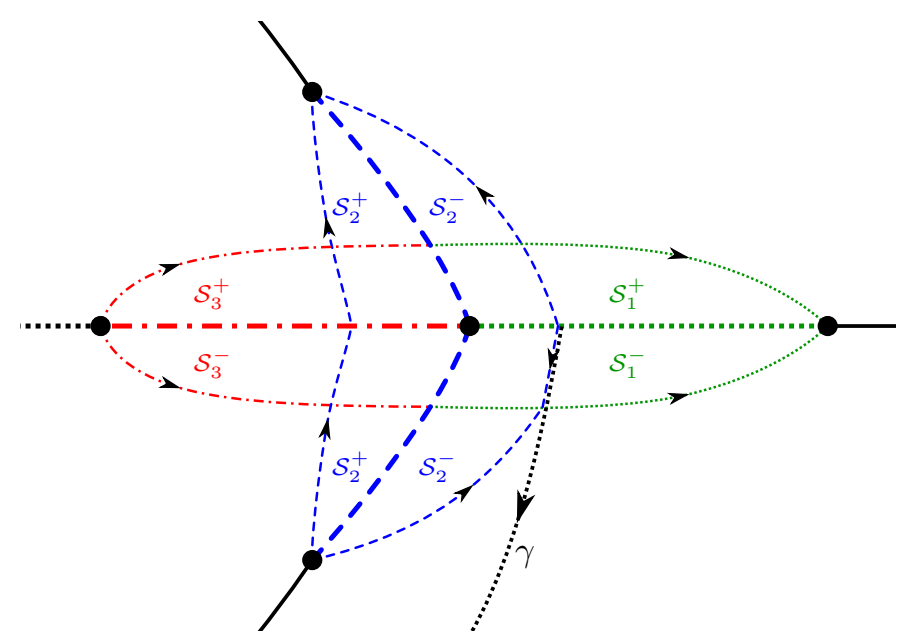

FIGURE 18. The opening of lenses in the supercritical case.

In other words, we define

$$
\boldsymbol{S}(z)=\boldsymbol{T}(z) \times \begin{cases}\boldsymbol{I}, & z \in \mathbb{C} \backslash \mathcal{S}_{1} \cup \mathcal{S}_{2} \cup \mathcal{S}_{3}, \\ \boldsymbol{I} \pm e^{N \Phi_{1}} \boldsymbol{M}_{21}, & z \in \mathcal{S}_{1}^{\mp} \backslash \mathcal{S}_{2}, \\ \boldsymbol{I} \pm e^{N \Phi_{2}} \boldsymbol{M}_{31}, & z \in \mathcal{S}_{2}^{\mp} \backslash\left(\mathcal{S}_{1} \cup \mathcal{S}_{3}\right), \\ \boldsymbol{I} \pm e^{N \Phi_{3}} \boldsymbol{M}_{23}, & z \in \mathcal{S}_{3}^{\mp} \backslash \mathcal{S}_{2} . \\ \left(\boldsymbol{I}+e^{N \Phi_{2}} \boldsymbol{M}_{31}\right)\left(\boldsymbol{I}-e^{N \Phi_{1}} \boldsymbol{M}_{21}\right), & z \in \mathcal{S}_{1}^{+} \cap \mathcal{S}_{2}^{-}, \\ \left(\boldsymbol{I}-e^{N \Phi_{2}} \boldsymbol{M}_{31}\right)\left(\boldsymbol{I}-e^{N \Phi_{3}} \boldsymbol{M}_{23}\right), & z \in \mathcal{S}_{2}^{+} \cap \mathcal{S}_{3}^{+}, \\ \left(\boldsymbol{I}-e^{N \Phi_{2}} \boldsymbol{M}_{31}\right)\left(\boldsymbol{I}+e^{N \Phi_{3}} \boldsymbol{M}_{23}\right), & z \in \mathcal{S}_{2}^{+} \cap \mathcal{S}_{3}^{-}, \\ \left(\boldsymbol{I}+e^{N \Phi_{2}} \boldsymbol{M}_{31}\right)\left(\boldsymbol{I}+e^{N \Phi_{1}} \boldsymbol{M}_{21}\right), & z \in \mathcal{S}_{1}^{-} \cap \mathcal{S}_{2}^{+} .\end{cases}
$$

With this choice, the jump matrix $\boldsymbol{J}_{S}$ for $\boldsymbol{S}$ becomes

$$
\boldsymbol{J}_{S}= \begin{cases}\boldsymbol{\sigma}_{3 j}, & z \in \Delta_{j}, j=1,2,3 \\ \boldsymbol{I}+e^{N \Phi_{1}} \boldsymbol{M}_{21}, & z \in \partial \mathcal{S}_{1} \\ \boldsymbol{I}+e^{N \Phi_{2}} \boldsymbol{M}_{31}, & z \in \partial \mathcal{S}_{2} \backslash\left(\gamma \cup \mathcal{S}_{3}\right) \\ \boldsymbol{I}+e^{N \Phi_{3}} \boldsymbol{M}_{23}, & z \in \partial \mathcal{S}_{3} \\ \boldsymbol{I}+e^{N \Phi_{2}}\left(\boldsymbol{M}_{31}+e^{N \Phi_{3}} \boldsymbol{M}_{21}\right), & z \in \partial \mathcal{S}_{2} \cap \mathcal{S}_{3}^{+} \\ \boldsymbol{I}+e^{N \Phi_{2}}\left(\boldsymbol{M}_{31}-e^{N \Phi_{3}} \boldsymbol{M}_{21}\right), & z \in \partial \mathcal{S}_{2} \cap \mathcal{S}_{3}^{-} \\ \boldsymbol{I}+e^{N \Phi_{2}}\left(\boldsymbol{M}_{32}+\left(e^{N \Phi_{1}}-1\right) \boldsymbol{M}_{31}\right), & z \in \partial \mathcal{S}_{2} \cap \gamma \\ \boldsymbol{J}_{T}, & \text { elsewhere. }\end{cases}
$$

Still, $\boldsymbol{S}(z)=I+\mathcal{O}\left(z^{-1}\right)$ as $z \rightarrow \infty$, and $\boldsymbol{S}(z)$ is bounded at all end points of the analytic arcs comprising $\Gamma_{S}=\Gamma_{T} \cup \partial \mathcal{S}_{1} \cup \partial \mathcal{S}_{2} \cup \partial \mathcal{S}_{3}$.

As before, we conclude that away from $\Delta_{j}$ 's the jump matrix $\boldsymbol{J}_{S}$ is exponentially close to $\boldsymbol{I}$, and this is true uniformly all the way up to $\Delta_{j}$ 's as long as we stay away from the end points $a_{j}, b_{j}$. 
6.5. Construction of the global parametrix. In order to deal with the jumps $\boldsymbol{J}_{S}=\boldsymbol{\sigma}_{3 j}$ on $\Delta_{j}, j=1,2,3$, see 6.29 , we will use a model matrix ("global parametrix") $\boldsymbol{F}$ solving the following RHP 3 .

- $\boldsymbol{F}: \mathbb{C} \backslash \Gamma_{F} \stackrel{\text { def }}{=} \Delta_{1} \cup \Delta_{2} \cup \Delta_{3} \rightarrow \mathbb{C}^{3 \times 3}$ is analytic;

- $\boldsymbol{F}_{+}(z)=\boldsymbol{F}_{-}(z) \boldsymbol{J}_{F}(z), z \in \Gamma_{F}$, where $\boldsymbol{J}_{F}=\boldsymbol{\sigma}_{3, j}$ on $\Delta_{j}$;

- $\boldsymbol{F}(z)=\mathcal{O}\left((z-q)^{-1 / 4}\right)$, as $z \rightarrow q \in\left\{a_{1}, a_{2}, b_{1}, b_{2}\right\}$.

- $\boldsymbol{F}(z)=\boldsymbol{I}+\mathcal{O}\left(z^{-1}\right)$ as $z \rightarrow \infty$.

The uniqueness of the solution of this problem follows from standard arguments. We construct the global parametrix using the general approach with meromorphic differentials and abelian integrals on the Riemann surface $\mathcal{R}$, see e.g. 31. Namely, for $i=1,2,3$, let $\eta_{i}$ be the meromorphic differential on $\mathcal{R}$ uniquely defined by the following conditions:

- its only poles (all simple) are the branch points $a_{1}^{(1)}, a_{2}^{(1)}, b_{1}^{(1)}, b_{2}^{(1)}$ and the points at infinity $\infty^{(j)}, j \in\{1,2,3\}, j \neq i$;

- the residues at these poles are

$$
\begin{aligned}
& \operatorname{Res}\left(\eta_{i}, a_{j}^{(1)}\right)=\operatorname{Res}\left(\eta_{i}, b_{j}^{(1)}\right)=-\frac{1}{2}, \quad j=1,2 . \\
& \operatorname{Res}\left(\eta_{i}, \infty^{(j)}\right)=1, \quad j \in\{1,2,3\}, \quad j \neq i .
\end{aligned}
$$

Since the sum of residues is zero and the genus of $\mathcal{R}$ is also zero, conditions above determine each meromorphic differential $\eta_{i}$ uniquely. Moreover, in virtue of $(6.30)-$ 6.31 we get that for any loop $\zeta \subset \mathcal{R}_{j}, j=1,2,3$, the differential $\eta$ satisfies

$$
\int_{\zeta} \eta_{i} \equiv 0 \bmod (2 \pi i)
$$

We define the corresponding abelian integrals

$$
u_{i}(p) \stackrel{\text { def }}{=} \int_{\infty^{(i)}}^{p} \eta_{i}, \quad p \in \mathcal{R}, \quad i=1,2,3
$$

specifying in each case the path of integration is as follow 4

(i) For $i=1,2,3$, if $p \in \mathcal{R}_{i}$, then for $u_{i}(p)$ we integrate along any path entirely contained in $\mathcal{R}_{i}$

(ii) For $u_{1}(p)$,

- if $p \in \mathcal{R}_{2}$, then the path starting in $\mathcal{R}_{1}$, enters $\mathcal{R}_{2}$ through the cut $\Delta_{1-}^{(1)}$ and stays in $\mathcal{R}_{2}$;

- if $p \in \mathcal{R}_{3}$, then the path starting in $\mathcal{R}_{1}$, enters $\mathcal{R}_{3}$ through the cut $\Delta_{2-}^{(1)}$ and stays in $\mathcal{R}_{3}$;

(iii) For $u_{2}(p)$,

- if $p \in \mathcal{R}_{1}$, then the path starting in $\mathcal{R}_{2}$, enters $\mathcal{R}_{1}$ through the cut $\Delta_{1+}^{(2)}$ and stays in $\mathcal{R}_{1}$;

- if $p \in \mathcal{R}_{3}$, then the path used to define $u_{2}(p)$ on $\mathcal{R}_{1}$, is continued, enters $\mathcal{R}_{3}$ from $\mathcal{R}_{1}$ through the cut $\Delta_{2-}^{(1)}$ and stays in $\mathcal{R}_{3} ;$

(iv) For $u_{3}(p)$,

\footnotetext{
${ }^{3}$ Recall that $\Delta_{1}$ and $\Delta_{3}$ have the orientation of $\mathbb{R}$, while $\Delta_{2}$ is oriented upwards, from $a_{2}$ to $b_{2}$.

${ }^{4}$ For the sheet structure of $\mathcal{R}$, see Figure 7
} 
- if $p \in \mathcal{R}_{1}$, then the path starting in $\mathcal{R}_{3}$, enters $\mathcal{R}_{1}$ through the cut $\Delta_{2+}^{(3)}$ and stays in $\mathcal{R}_{1}$;

- if $p \in \mathcal{R}_{2}$, then the path used to define $u_{3}(p)$ on $\mathcal{R}_{1}$, is continued, enters $\mathcal{R}_{2}$ from $\mathcal{R}_{1}$ the cut $\Delta_{1-}^{(1)}$ and stays in $\mathcal{R}_{2}$.

The selection of paths is determined by the structure of the jump matrix $\boldsymbol{J}_{F}$. Observe that in virtue of 6.32 functions $u_{i}$ are well defined modulo $2 \pi i$. Furthermore, they satisfy, for instance, for $p \in \Delta_{j}^{(1)}$ and $j=1,2$,

$$
\begin{array}{rlrl}
u_{1+}(p)-u_{1-}(p) & \equiv 2 \pi i \operatorname{Res}\left(\eta_{1}, b_{j}^{(1)}\right) & & \bmod (2 \pi i) \\
& \equiv \pi i \quad \bmod (2 \pi i), \quad i=1,2,3,
\end{array}
$$

with analogous additive jump conditions for $u_{2}$ on $\Delta_{1} \cap \Delta_{3}$ and $u_{3}$ on $\Delta_{2} \cap \Delta_{3}$. Moreover, from 6.30 we get that they admit the (multivalued) expansion

$$
u_{i}(z)=-\frac{1}{4} \log (z-q)+\mathcal{O}(1), \quad z \rightarrow q \in\left\{a_{1}, a_{2}, b_{1}, b_{2}\right\} .
$$

Now we define

$$
f_{i}(p) \stackrel{\text { def }}{=} e^{u_{i}(p)}, \quad p \in \mathcal{R},
$$

and

$$
\left.f_{i j} \stackrel{\text { def }}{=} f_{i}\right|_{\mathcal{R}_{j}}, \quad i, j=1,2,3 .
$$

Notice that by construction, $f_{i 1}, f_{i 2}$ and $f_{i 3}$ are holomorphic on $\mathbb{C} \backslash\left(\Delta_{1} \cup \Delta_{2}\right)$, $\mathbb{C} \backslash\left(\Delta_{1} \cup \Delta_{3}\right)$ and $\mathbb{C} \backslash\left(\Delta_{2} \cup \Delta_{3}\right)$, respectively. Furthermore, in virtue of $(6.33)$ they satisfy the relations

$$
\begin{aligned}
& \left(f_{i 1}\right)_{+}(z)=-\left(f_{i, j+1}\right)_{-}(z), \quad z \in \Delta_{j}, j=1,2, \\
& \left(f_{i 3}\right)_{+}(z)=\left(f_{i, 2}\right)_{-}(z), \quad z \in \Delta_{3},
\end{aligned}
$$

and from their definition it follows that also

$$
\begin{aligned}
& \left(f_{i 1}\right)_{-}(z)=\left(f_{i, j+1}\right)_{+}(z), \quad z \in \Delta_{j}, j=1,2, \\
& \left(f_{i 3}\right)_{+}(z)=\left(f_{i, 3}\right)_{-}(z), \quad z \in \Delta_{1}, \\
& \left(f_{i 2}\right)_{+}(z)=\left(f_{i, 2}\right)_{-}(z), \quad z \in \Delta_{2}, \\
& \left(f_{i 1}\right)_{+}(z)=\left(f_{i, 1}\right)_{-}(z), \quad z \in \Delta_{3}, \\
& \left(f_{i 3}\right)_{-}(z)=\left(f_{i, 2}\right)_{+}(z), \quad z \in \Delta_{3} .
\end{aligned}
$$

Moreover, 6.34 gives us

$$
f_{i j}(z)=(z-q)^{-1 / 4}(1+o(1)), \quad z \rightarrow q,
$$

whenever $q \in\left\{a_{1}, a_{2}, b_{1}, b_{2}\right\}$ is a singularity of $f_{i j}, j=1,2,3$. Finally, from the definition of $u_{i}$ 's we conclude that

$$
f_{i i}(z)=1+\mathcal{O}\left(z^{-1}\right), \quad z \rightarrow \infty, \quad i=1,2,3,
$$

while from 6.31) we get also that

$$
f_{i j}(z)=\frac{1}{z}+\mathcal{O}\left(z^{-2}\right), \quad z \rightarrow \infty, \quad i, j \in\{1,2,3\}, \quad i \neq j .
$$

Summarizing, conditions 6.35-6.37, show that

$$
\boldsymbol{F} \stackrel{\text { def }}{=}\left(f_{i j}\right)_{i, j=1}^{3}
$$

is the unique solution of the RHP stated at the beginning of this section. 


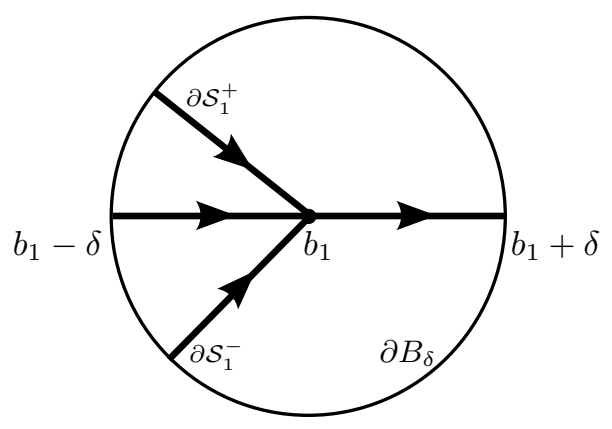

Figure 19. Local analysis at $b_{1}$.

Remark 6.2. Notice that the $\mathrm{RH}$ problem for $\boldsymbol{F}$ easily implies that $\operatorname{det}(\boldsymbol{F}(z)) \equiv 1$ on $\mathbb{C}$, so that $\boldsymbol{F}$ is invertible everywhere on the plane (which is used in the standard argument proving the uniqueness of the solution $\boldsymbol{F}$ ). Moreover, by (6.15),

$$
\sigma_{3, j}^{-1}=\sigma_{3, j}^{T}, \quad j=1,2,3,
$$

so that $\left(\boldsymbol{F}^{-1}\right)^{T}$ satisfies the same RHP than $\boldsymbol{F}$. By uniqueness, we conclude that

$$
\boldsymbol{F}^{-1}(z)=\boldsymbol{F}^{T}(z), \quad z \in \mathbb{C} .
$$

6.6. Parametrices near branch points. Our construction shows that the matrixvalued function $\boldsymbol{F}$ defined in the previous section is invertible on $\mathbb{C} \backslash \Gamma_{S}$, and $\boldsymbol{F}^{-1} \boldsymbol{S}$ is analytic in $\mathbb{C} \backslash \Gamma_{S}$ and has jumps exponentially close to $\boldsymbol{I}$ everywhere on $\Gamma_{S}$ except at the branch points $a_{1}, a_{2}, b_{1}, b_{2}$. In order to get this proximity to $\boldsymbol{I}$ uniform on all contours we have to replace $\boldsymbol{F}$ by a different model, a local parametrix. Its construction, nowadays standard, is done in terms of Airy functions.

We describe here only the construction at the end point $b_{1}$, since for the rest of branch points it is similar.

Consider a small fixed disk, $B_{\delta}=B_{\delta}\left(b_{1}\right) \stackrel{\text { def }}{=}\left\{z \in \mathbb{C}:\left|z-b_{1}\right|<\delta\right\}$, of center at $b_{1}$ and radius $\delta>0$ small enough so that $B_{\delta}$ does not contain any other branch point of $\mathcal{R}$ or $a_{*}$ (see Figure 19p. We look for $\boldsymbol{P}\left(\cdot ; b_{1}\right)$ holomorphic in $B_{\delta} \backslash\left(\mathbb{R} \cup \partial \mathcal{S}_{1}^{ \pm}\right)$, such that $\boldsymbol{P}_{+}\left(z ; b_{1}\right)=\boldsymbol{P}_{-}\left(z ; b_{1}\right) \boldsymbol{J}_{S}(z)$, where, by 3.26$)$ and $(6.29)$,

$$
\begin{aligned}
& \boldsymbol{J}_{S}(x)=\left(\begin{array}{ccc}
0 & 1 & 0 \\
-1 & 0 & 0 \\
0 & 0 & 1
\end{array}\right), \quad x \in B_{\delta} \cap \Delta_{1}=\left(b_{1}-\delta, b_{1}\right), \\
& \boldsymbol{J}_{S}(x)=\left(\begin{array}{ccc}
1 & e^{-N \Phi_{1}(x)} & 0 \\
0 & 1 & 0 \\
0 & 0 & 1
\end{array}\right), \quad x \in\left(b_{1}, b_{1}+\delta\right), \\
& \boldsymbol{J}_{S}(z)=\left(\begin{array}{ccc}
1 & 0 & 0 \\
e^{N \Phi_{1}(z)} & 1 & 0 \\
0 & 0 & 1
\end{array}\right), \quad z \in B_{\delta} \cap \partial \mathcal{S}_{1}^{ \pm},
\end{aligned}
$$

and $\boldsymbol{P}\left(\cdot ; b_{1}\right)$ is bounded as $z \rightarrow b_{1}, z \in \mathbb{C} \backslash\left(\mathbb{R} \cup \partial \mathcal{S}_{1}^{ \pm}\right)$.

With these conditions, by (6.16), matrix

$$
\widehat{\boldsymbol{P}}\left(z ; b_{1}\right) \stackrel{\text { def }}{=} \boldsymbol{P}\left(z ; b_{1}\right) \operatorname{diag}\left(e^{-N \Phi_{1}(z) / 2}, e^{N \Phi_{1}(z) / 2}, 1\right)
$$


has constant jumps, $\widehat{\boldsymbol{P}}_{+}\left(z ; b_{1}\right)=\widehat{\boldsymbol{P}}_{-}\left(z ; b_{1}\right) \boldsymbol{J}_{\widehat{P}}(z)$, with

$$
\begin{aligned}
& \boldsymbol{J}_{\widehat{P}}(x)=\left(\begin{array}{ccc}
0 & 1 & 0 \\
-1 & 0 & 0 \\
0 & 0 & 1
\end{array}\right), \quad x \in B_{\delta} \cap \Delta_{1}=\left(b_{1}-\delta, b_{1}\right), \\
& \boldsymbol{J}_{\widehat{P}}(x)=\left(\begin{array}{lll}
1 & 1 & 0 \\
0 & 1 & 0 \\
0 & 0 & 1
\end{array}\right), \quad x \in\left(b_{1}, b_{1}+\delta\right), \\
& \boldsymbol{J}_{\widehat{P}}(z)=\left(\begin{array}{lll}
1 & 0 & 0 \\
1 & 1 & 0 \\
0 & 0 & 1
\end{array}\right), \quad z \in B_{\delta} \cap \partial \mathcal{S}_{1}^{ \pm}
\end{aligned}
$$

and again $\widehat{\boldsymbol{P}}$ is bounded as $z \rightarrow b_{1}, z \in \mathbb{C} \backslash\left(\mathbb{R} \cup \partial \mathcal{S}_{1}^{ \pm}\right)$.

Since we additionally need that, as $n \rightarrow \infty$,

$$
\boldsymbol{P}\left(z ; b_{1}\right)=(\boldsymbol{I}+\mathcal{O}(1 / N)) \boldsymbol{F}(z) \quad z \in \partial B_{\delta} \backslash\left(\mathbb{R} \cup \partial \mathcal{S}_{1}^{ \pm}\right),
$$

we follow a well-known scheme, and build $\widehat{\boldsymbol{P}}\left(\cdot ; b_{1}\right)$ in the form

$$
\widehat{\boldsymbol{P}}\left(z ; b_{1}\right)=\boldsymbol{E}(z) \boldsymbol{\Psi}\left(N^{2 / 3} \varphi(z)\right),
$$

where

$$
\boldsymbol{E}(z)=\boldsymbol{F}(z)\left(\begin{array}{ccc}
\sqrt{\pi} & -\sqrt{\pi} & 0 \\
-i \sqrt{\pi} & -i \sqrt{\pi} & 0 \\
0 & 0 & 1
\end{array}\right)\left(\begin{array}{ccc}
n^{1 / 6} \varphi^{1 / 4}(z) & 0 & 0 \\
0 & n^{-1 / 6} \varphi^{-1 / 4}(z) & 0 \\
0 & 0 & 1
\end{array}\right)
$$

and

$$
\varphi(z)=\left[\frac{3}{2} \Phi_{1}(z)\right]^{2 / 3}
$$

is a biholomorphic (conformal) map of a neighborhood of $b_{1}$ onto a neighborhood of the origin such that $\varphi(z)$ is real and positive for $z>b_{1}$, see (3.4)-3.6) and (3.23).

We may deform the contours $\mathcal{S}_{1}^{ \pm}$near $b_{1}$ in such a way that $\varphi$ maps $\mathcal{S}_{1}^{ \pm} \cap B_{\delta}$ onto the rays with angles $\frac{2 \pi}{3}$ and $-\frac{2 \pi}{3}$, respectively.

Matrix $\boldsymbol{\Psi}$ is built using a Riemann-Hilbert problem for the Airy functions, as described for instance in [28, page 253]. We recall the main steps for later reference.

First consider a $2 \times 2$ matrix $\boldsymbol{K}$ solving the following RHP.

- $\boldsymbol{K}: \mathbb{C} \backslash \Gamma_{K} \rightarrow \mathbb{C}^{2 \times 2}$ is analytic, where $\Gamma_{K}=\mathbb{R} \cup\left[0, e^{i 2 \pi i / 3} \infty\right) \cup\left[0, e^{-2 \pi i / 3} \infty\right)$;

- $\boldsymbol{K}$ has continuous boundary values $\boldsymbol{\Psi}_{ \pm}$on $\Gamma_{\Psi}$, and $\boldsymbol{\Psi}_{+}(z)=\boldsymbol{\Psi}_{-}(z) \boldsymbol{J}_{\Psi}(z)$, $z \in \Gamma_{\Psi}$, with

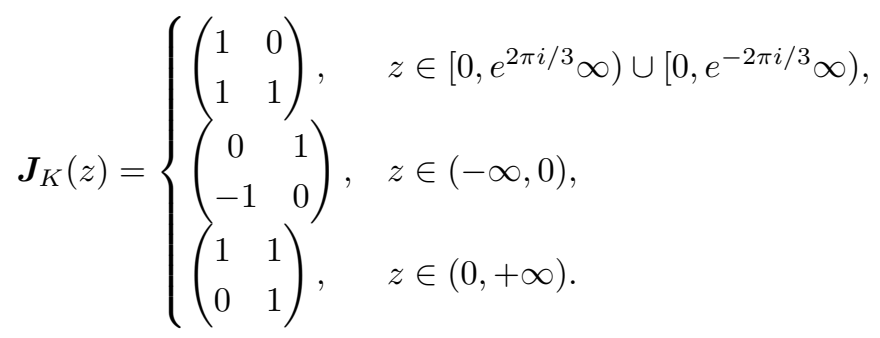

- As $z \rightarrow \infty$ with $-\pi<\arg z<\pi$,

$\boldsymbol{K}(z)=\left(I+\mathcal{O}\left(z^{-1}\right)\right) \operatorname{diag}\left(z^{-1 / 4}, z^{1 / 4}\right) \frac{1}{\sqrt{2}}\left(\begin{array}{cc}1 & i \\ i & 1\end{array}\right) \operatorname{diag}\left(e^{-\frac{2}{3} z^{3 / 2}}, e^{\frac{2}{3} z^{3 / 2}}\right) ;$ 
- $\boldsymbol{K}$ remains bounded as $z \rightarrow \infty$ along $\mathbb{C} \backslash \Gamma_{\Psi}$.

We put

$$
y_{0}(s)=\operatorname{Ai}(s), \quad y_{1}(s)=\omega \operatorname{Ai}(\omega s), \quad y_{2}(s)=\omega^{2} \operatorname{Ai}\left(\omega^{2} s\right), \quad \omega=e^{2 \pi i / 3},
$$

where Ai is the usual Airy function. Then $\boldsymbol{K}$ is obtained explicitly as

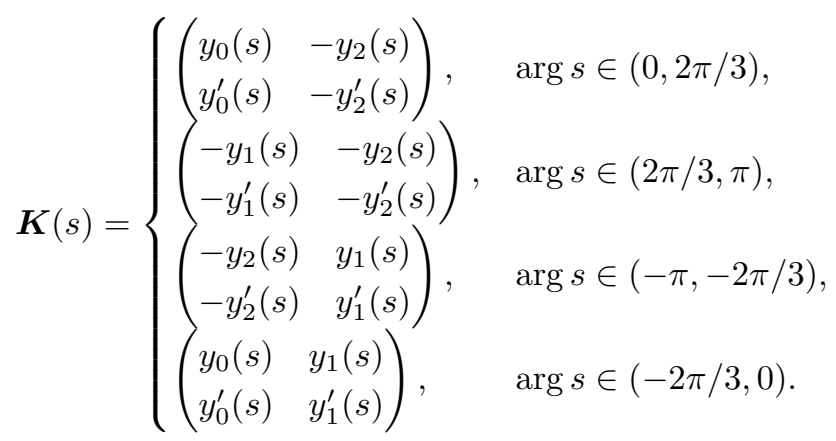

Then we take the $3 \times 3$ matrix $\boldsymbol{\Psi}$ as

$$
\boldsymbol{\Psi}(s)=\left(\begin{array}{c:c}
\boldsymbol{K}(s) & \mathbf{0} \\
\hdashline \mathbf{0} & 1^{-}
\end{array}\right) .
$$

As we said, the construction of the rest of the local parametrices $\boldsymbol{P}(\cdot ; q), q \in$ $\left\{a_{1}, a_{2}, b_{2}\right\}$, follows the same scheme, nowadays standard.

6.7. Final transformation. We define the matrix valued function $\boldsymbol{R}$ as

$\boldsymbol{R}(z) \stackrel{\text { def }}{=} \begin{cases}\boldsymbol{S}(z) \boldsymbol{P}^{-1}(z ; q), & \text { in the neighborhoods } B_{\delta}(q) \text { around } q \in\left\{a_{1}, a_{2}, b_{1}, b_{2}\right\}, \\ \boldsymbol{S}(z) \boldsymbol{F}^{-1}(z), & \text { elsewhere. }\end{cases}$

The jump matrices of $\boldsymbol{S}$ and $\boldsymbol{F}$ coincide on $\Delta_{1} \cup \Delta_{2} \cup \Delta_{3}$ and the jump matrices of $\boldsymbol{S}$ and $\boldsymbol{P}$ coincide inside the four disks $B_{\delta}(q), q \in\left\{a_{1}, a_{2}, b_{1}, b_{2}\right\}$. It follows that $\boldsymbol{R}$ has an analytic continuation to the complex plane minus the contours $\Gamma_{R}$ shown in Figure 20. The matching conditions 6.39 shows that $\boldsymbol{P} \boldsymbol{F}^{-1}=\boldsymbol{I}+\mathcal{O}(1 / N)$ as $N \rightarrow \infty$, uniformly on $\bigcup_{q} \partial B_{\delta}(q)$, Thus

$\boldsymbol{R}_{+}(z)=\boldsymbol{R}_{-}(z)(\boldsymbol{I}+\mathcal{O}(1 / N)), \quad$ as $N \rightarrow \infty$, uniformly on $\bigcup_{q \in\left\{a_{1}, a_{2}, b_{1}, b_{2}\right\}} \partial B_{\delta}(q)$, while on the rest of the contours of $\Gamma_{R}$, for some $c>0$,

$$
\boldsymbol{R}_{+}(z)=\boldsymbol{R}_{-}(z)\left(\boldsymbol{I}+\mathcal{O}\left(e^{-c N}\right)\right), \quad \text { as } N \rightarrow \infty .
$$

Since $\boldsymbol{R}(z) \rightarrow \boldsymbol{I}$ as $z \rightarrow \infty$, standard arguments yield that

$$
\boldsymbol{R}(z)=\boldsymbol{I}+\mathcal{O}(1 / N) \text { as } N \rightarrow \infty,
$$

uniformly in $z \in \mathbb{C} \backslash \Gamma_{R}$.

\section{Steepest DESCEnt ANALysis For General $\alpha$}

All transformation described in Section 6 were carried out under the assumption 6.1. Now we extend our conclusions to arbitrary sequences of $\alpha_{N}$ satisfying

$$
\alpha_{N} \rightarrow \alpha_{0} \in(0,1 / 2) \backslash\left\{\alpha_{c}\right\} .
$$

We also assume that $N$ is chosen large enough so that $\alpha_{N}$ and $\alpha_{0}$ belong to the same connected component of $(0,1 / 2) \backslash\left\{\alpha_{c}\right\}$. 


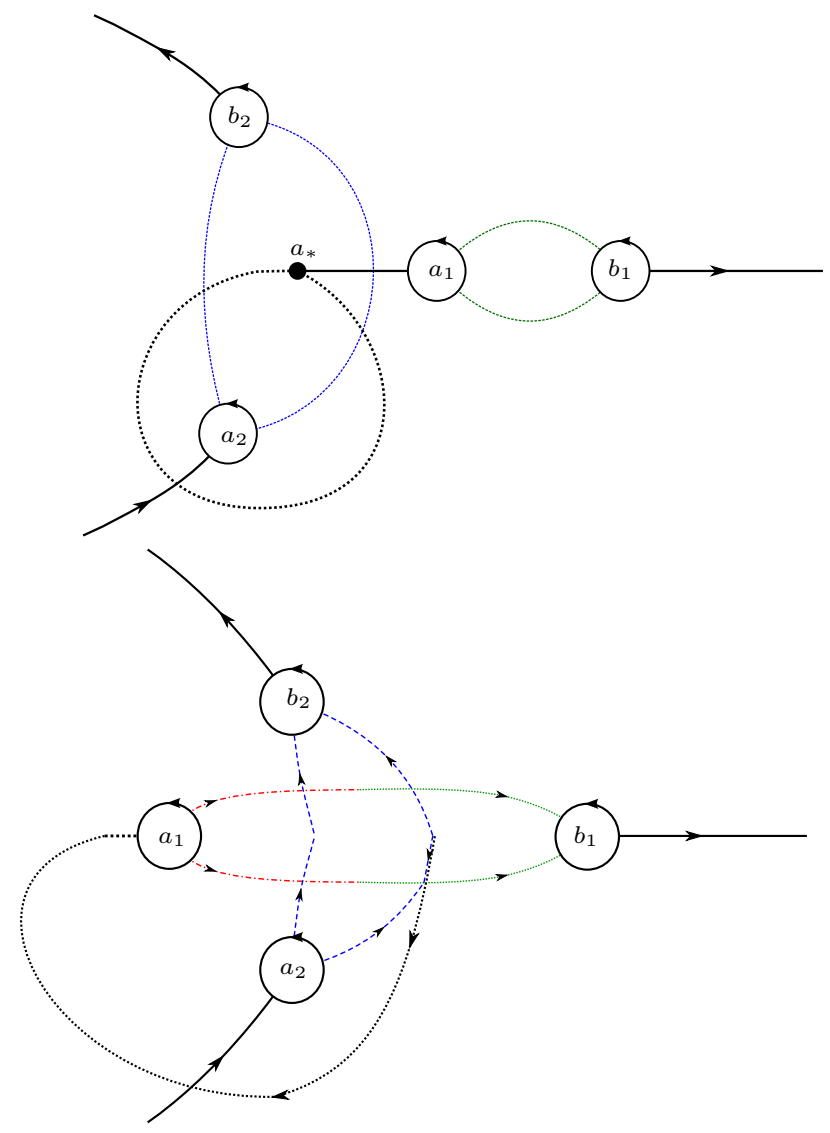

Figure 20. Pictorial representation of the contours $\Gamma_{R}$ in the sub(top) and super-critical (bottom) regimes.

Starting with the first Riemann-Hilbert problem $\boldsymbol{Y}=\boldsymbol{Y}_{\alpha_{N}}$, we make the first transformation $\boldsymbol{Y}_{\alpha_{N}} \mapsto \boldsymbol{X}_{\alpha_{N}}$ as in 6.1.

The second transformation $\boldsymbol{X}_{\alpha_{N}} \mapsto \boldsymbol{T}_{\alpha_{N}}$ is also as before, that is, as in 6.4), taking into account that all required quantities will depend on $\alpha_{N}$. That is, for the given $\alpha_{N}$, we define $\xi_{k}=\xi_{k, \alpha_{N}}$ as the solutions of the spectral curve (2.7) and asymptotics (3.1) with $\alpha=\alpha_{N}$, then we define the corresponding $g$-functions $g_{k}=g_{k, \alpha_{N}}$ and $r$-constants $r_{k}=r_{k, \alpha_{N}}$ as in (3.19) and (3.22), respectively.

With such choices, all the identities used for the $g$-functions remain valid, taking into account that $\alpha=\alpha_{N}$ and also that the $\Phi$-functions in $3.23-(3.25)$ will be $\alpha_{N}$-dependent.

At this stage, the jump matrix $\boldsymbol{J}_{T}$ remains as in $(6.12)$, stressing once again that the $g$-functions now depend on $\alpha_{N}$. Thus, we can open lenses as in 6.18) (in the subcritical case) or as in 6.28 (in the supercritical case). At this stage, we also fix sufficiently small disks $B_{\delta}\left(a_{k}\right), B_{\delta}\left(b_{k}\right)$ for $a_{k}=a_{k}\left(\alpha_{0}\right)$ and $b_{k}=b_{k}\left(\alpha_{0}\right)$ and denote their union by $B$. The precise choice of $\delta$ is determined later in the construction of the local parametrix, but we anticipate that it will be possible to choose $\delta$ to be depending on $\alpha_{0}$ but not on $\alpha_{N}$. 
The shape of the lenses $\mathcal{S}_{j}^{ \pm}$does also depend on $\alpha_{N}$. However, continuity and convergence of all the $\alpha_{N}$-dependent quantities guarantee that we can choose the lenses so that

- the endpoints $a_{1}=a_{1}\left(\alpha_{N}\right)$ (in the subcritical case), $a_{2}=a_{2}\left(\alpha_{N}\right), b_{1}=$ $b_{1}\left(\alpha_{N}\right)$ and $b_{2}=b_{2}\left(\alpha_{N}\right)$ all belong to $B$,

- the sets $\mathcal{S}_{j}^{ \pm} \backslash B$ are independent of $\alpha_{N}$,

and in such a way that the uniform version of the inequalities 6.22 (in the subcritical case) or 6.27) (in the supercritical case) hold true on the lenses away from the endpoints, namely

$$
\operatorname{Re} \Phi_{j, \alpha_{0}}(z) \leq-\rho<0, \quad \operatorname{Re} \Phi_{j, \alpha_{N}}(z) \leq-\rho<0, \quad z \in \partial \mathcal{S}_{j}^{ \pm} \backslash B,
$$

for every $n, N$ sufficiently large and some fixed $\rho>0$ that does not depend on the parameters $\alpha_{N}$ but only on $\alpha_{0}$. We stress that the sets $\mathcal{S}_{j}^{ \pm} \backslash B$ appearing above were chosen to be $\alpha_{N}$-independent.

After the opening of lenses, the obtained matrix $\mathbf{S}$ has jumps as in 6.20 (in the subcritical case) or (6.29) (in the supercritical case). In virtue of (7.1), at this stage we can be sure that $\boldsymbol{J}_{S}$ decays exponentially to the identity uniformly away from $B$ and from the sets $\Delta_{k}=\Delta_{k}\left(\alpha_{N}\right)$.

For the construction of the parametrix, we observe that $\boldsymbol{J}_{S}$ has constant jumps on $\Delta_{k}\left(\alpha_{N}\right)$. Thus, for any $\alpha_{N}$ the global matrix $\boldsymbol{F}$ we consider is the one constructed in Section 6.5. but for the choice $\alpha=\alpha_{0}$. Although $\boldsymbol{J}_{F}$ does not coincide with $\boldsymbol{J}_{S}$ close to the varying endpoints $a_{k}\left(\alpha_{N}\right)$ and $b_{k}\left(\alpha_{N}\right)$, these points are assumed to be on $B$ (by the construction of $B$ ), so this local inconsistency of $\boldsymbol{J}_{F}$ with $\boldsymbol{J}_{S}$ will anyway be solved by the local parametrix.

In each of the connected components of $B$, we need to construct the local parametrix $\boldsymbol{P}$ that will depend on $\alpha_{N}$. We focus on the construction connected component $B\left(b_{1}\right)$, recalling that $b_{1}=b_{1}\left(\alpha_{0}\right)$. The overall method is the same as the one explained in Section 6.6. but carried out for $\alpha=\alpha_{N}$. The main differences are explained next.

The neighborhood $B\left(b_{1}\right)$ can be chosen to be a disc centered at $b_{1}=b_{1}\left(\alpha_{0}\right)$ but not at $b_{1}\left(\alpha_{N}\right)$. This imposes no extra difficulty.

The conformal map $\varphi=\varphi_{\alpha_{N}}$ in 6.42 is now varying with $\alpha_{N}$. At any rate, it is easy to see that

$$
\varphi_{\alpha_{N}} \rightarrow \varphi_{\alpha_{0}}
$$

locally uniformly. The function $\Phi_{1}=\Phi_{1, \alpha_{N}}$ satisfies

$$
\Phi_{1, \alpha_{N}}(z)=z^{3}+\mathcal{O}(1), \quad z \rightarrow \infty
$$

as can be seen from 3.22 and 3.26 .

From the expansion above, the following claim follows. For each $\alpha_{N}$, there exists an $\operatorname{arc} \Gamma\left(\alpha_{N}\right)$ starting at the origin, extending to $\infty$ with angle $2 \pi / 3$, and such that $\varphi_{\alpha_{N}}$ maps $\partial \mathcal{S}^{+} \cap B\left(b_{1}\right)$ onto $\Gamma_{N}$, and $\Gamma\left(\alpha_{0}\right)$ reduces to a half line ray.

Having the construction above in mind, we consider the RHP for $\boldsymbol{K}$, replacing in 6.43 the arcs $\left[0, e^{2 \pi i / 3} \infty\right)$ and $\left[0, e^{-2 \pi i / 3} \infty\right)$ by $\Gamma\left(\alpha_{N}\right)$ and its symmetric $\Gamma\left(\alpha_{N}\right)^{*}$ under complex conjugation. The solution $\boldsymbol{K}=\boldsymbol{K}_{\alpha_{N}}$ to this RHP can be constructed in a formula very much like (6.44), replacing the angular sectors by the sectors determined by $\Gamma\left(\alpha_{N}\right)$, its complex conjugate and the real axis. In particular, as it follows from their explicit formulas in terms of Airy functions, the 
convergence

$$
\boldsymbol{K}_{\alpha_{N}} \rightarrow \boldsymbol{K}_{\alpha_{0}}
$$

holds true uniformly in compacts of $\mathbb{C} \backslash\left(\mathbb{R} \cup\left[0, e^{2 \pi i / 3} \infty\right) \cup\left[0, e^{-2 \pi i / 3} \infty\right)\right)$.

Once the matrix $K_{\alpha_{N}}$ is constructed, we then obtain $\boldsymbol{\Phi}_{\alpha_{N}}$ as in 6.45 , consider the parametrix $\widehat{\boldsymbol{P}}_{\alpha_{N}}$ as in 6.40, and then obtain $\boldsymbol{P}_{\alpha_{N}}$ through 6.39), where we stress that $\boldsymbol{F}=\boldsymbol{F}_{\alpha_{0}}$ does not depend on $\alpha_{N}$.

Once the parametrices $\boldsymbol{P}_{\alpha_{N}}$ and $\boldsymbol{F}_{\alpha_{0}}$ are constructed, we define $R=R_{\alpha_{N}}$ in a similar way as in 6.46 , taking now the form

$$
\boldsymbol{R}_{\alpha_{N}}(z) \stackrel{\text { def }}{=} \begin{cases}\boldsymbol{S}_{\alpha_{N}}(z) \boldsymbol{P}_{\alpha_{N}}^{-1}(z), & z \in B, \\ \boldsymbol{S}_{\alpha_{N}}(z) \boldsymbol{F}_{\alpha_{0}}^{-1}(z), & \text { elsewhere. }\end{cases}
$$

and the analysis follows exactly as in Section 6.7 in particular leading to

$$
\boldsymbol{R}_{\alpha_{N}}(z)=I+\mathcal{O}(1 / N) \text { as } N \rightarrow \infty,
$$

uniformly for $z \in \mathbb{C} \backslash \Gamma_{R}$, where we also call the reader's attention to the fact that $\Gamma_{R}$ does not depend on $\alpha_{N}$.

\section{Asymptotics}

Now we can unravel all transformations and find the desired asymptotic expressions.

Proof of Theorem 2.7. For $z \in \mathbb{C} \backslash\left(\mathcal{S}_{1}^{ \pm} \cup \mathcal{S}_{2}^{ \pm} \cup \mathcal{U}\right)$, away from the contours $\Gamma_{R}$ depicted on Figure 20, we have

$$
\begin{aligned}
\boldsymbol{Y}(z)=\operatorname{diag}\left(e^{N r_{1}}, e^{N r_{2}}, e^{N r_{3}}\right)(\boldsymbol{I}+\mathcal{O}(1 / N)) \boldsymbol{F}(z) \\
\quad \times \operatorname{diag}\left(e^{-N\left(g_{1}(z)-\frac{2}{3} z^{3}\right)}, e^{-N\left(g_{2}(z)+\frac{1}{3} z^{3}\right)}, e^{-N\left(g_{3}(z)+\frac{1}{3} z^{3}\right)}\right) .
\end{aligned}
$$

Since the polynomial $P_{n, m}$ coincides with the $(1,1)$ entry of the matrix $\boldsymbol{Y}$, we have

$$
\begin{aligned}
P_{n, m}(z) & =\left(\begin{array}{lll}
1 & 0 & 0
\end{array}\right) \boldsymbol{Y}(z)\left(\begin{array}{l}
1 \\
0 \\
0
\end{array}\right) \\
& =e^{N\left(-g_{1}(z)+\frac{2}{3} z^{3}+r_{1}\right)}\left(\begin{array}{lll}
1 & 0 & 0
\end{array}\right)(\boldsymbol{I}+\mathcal{O}(1 / N)) \boldsymbol{F}(z)\left(\begin{array}{l}
1 \\
0 \\
0
\end{array}\right) \\
& =f_{11}(z) e^{N\left(-g_{1}(z)+\frac{2}{3} z^{3}+r_{1}\right)}(1+\mathcal{O}(1 / N)),
\end{aligned}
$$

which gives us the expression of Theorem 2.7 for $z \in \mathbb{C} \backslash\left(\mathcal{S}_{1}^{ \pm} \cup \mathcal{S}_{2}^{ \pm} \cup \mathcal{U}\right)$. Note that in the varying case $\alpha_{N}=n / N$, the formula above (and all the calculations below) are still the same with $g_{1}=g_{\alpha_{N}, 1}$, and the global parametrix is constructed with $f_{1 j}$ 's for the limiting value $\alpha=\lim \alpha_{N}$.

Furthermore, for $z \in \mathcal{U} \backslash\left(\mathcal{S}_{1}^{ \pm} \cup \mathcal{S}_{2}^{ \pm}\right)$,

$$
\begin{aligned}
\boldsymbol{Y}(z)=\operatorname{diag}\left(e^{N r_{1}}, e^{N r_{2}}, e^{N r_{3}}\right)(\boldsymbol{I}+\mathcal{O}(1 / N)) \boldsymbol{F}(z) \\
\quad \times \operatorname{diag}\left(e^{-N\left(g_{1}(z)-\frac{2}{3} z^{3}\right)}, e^{-N\left(g_{2}(z)+\frac{1}{3} z^{3}\right)}, e^{-N\left(g_{3}(z)+\frac{1}{3} z^{3}\right)}\right)\left(\boldsymbol{I}+\boldsymbol{M}_{32}\right),
\end{aligned}
$$

and we clearly obtain the same asymptotic expression for $P_{n, m}$ as before. Because the lenses $\mathcal{S}_{1}^{ \pm}$and $\mathcal{S}_{2}^{ \pm}$can be taken arbitrarily close to $\Delta_{1}$ and $\Delta_{2}$, this is enough to conclude Theorem 2.7 
Proof of Theorem 2.4 for $P_{n, m}$. Since $f_{11}$ does not vanish in $\mathbb{C} \backslash\left(\Delta_{1} \cup \Delta_{2}\right)$, we conclude from Theorem 2.7 that the zeros of $P_{n, m}$ asymptotically belong to $\Delta_{1} \cup \Delta_{2}$. Moreover, since domains $\mathcal{S}_{1}^{ \pm}$and $\mathcal{S}_{2}^{ \pm}$can be taken arbitrarily small, we conclude from 8.1) that

$$
\lim _{N \rightarrow \infty}\left|P_{n, m}(z)\right|^{1 / N}=e^{-\operatorname{Re}\left(g_{1}(z)-\frac{2}{3} z^{3}-r_{1}\right)}
$$

locally uniformly on any compact subset of $\mathbb{C} \backslash\left(\Delta_{1} \cup \Delta_{2}\right)$, where on the right-hand side above we use $g_{1}=g_{\alpha, 1}=\lim _{N} g_{\alpha_{N}, 1}$, the latter limit taken pointwise. Let us show that

$$
\operatorname{Re}\left(g_{1}(z)-\frac{2}{3} z^{3}-r_{1}\right)=U^{\mu_{1}+\mu_{2}}(z), \quad \mathbb{C} \backslash\left(\Delta_{1} \cup \Delta_{2}\right) .
$$

First of all, it is easy to observe that by definition 3.19 and asymptotics 3.22 ,

$$
g_{1}^{\prime}(z)-2 z^{2}=\xi_{1}(z)-2 z^{2}=-\frac{1}{z}+\mathcal{O}\left(1 / z^{2}\right), \quad z \rightarrow \infty .
$$

On the other hand, by (3.9)-3.10 and the definition of $\mu_{j}$ 's in 3.18,

$$
g_{1+}^{\prime}(z)-g_{1-}^{\prime}(z)=\xi_{1+}(z)-\xi_{1-}(z)= \begin{cases}2 \pi i \mu_{1}^{\prime}(z), & \text { if } z \in \Delta_{1}, \\ 2 \pi i \mu_{2}^{\prime}(z), & \text { if } z \in \Delta_{2},\end{cases}
$$

from which we conclude that

$$
g_{1}^{\prime}(z)-2 z^{2}=C^{\mu_{1}+\mu_{2}}(z), \quad \mathbb{C} \backslash\left(\Delta_{1} \cup \Delta_{2}\right) .
$$

Finally, 8.3 follows from the asymptotic formula 3.22 .

In particular, 8.2 can be written as

$$
\lim _{N \rightarrow \infty}\left|P_{n, m}(z)\right|^{1 / N}=e^{-U^{\mu_{1}+\mu_{2}}(z)}, \quad \mathbb{C} \backslash\left(\Delta_{1} \cup \Delta_{2}\right) .
$$

Since $\Delta_{1} \cup \Delta_{2}$ have empty interior and connected complement, this proves (see e.g. [44. Theorem 4.1]) the statement of Theorem 2.4 for the weak limit of $\nu\left(P_{n, m}\right)$.

Next we turn to the asymptotic analysis of the type I multiple orthogonal polynomials $A_{n, m}$ and $B_{n, m}$, satisfying the Definition 2.1. Recall that

$$
\begin{aligned}
& A_{n, m}(z)=\frac{1}{2 \pi i}\left(\begin{array}{lll}
0 & 1 & 0
\end{array}\right) \boldsymbol{Y}^{-1}(z)\left(\begin{array}{l}
1 \\
0 \\
0
\end{array}\right), \\
& B_{n, m}(z)=\frac{1}{2 \pi i}\left(\begin{array}{lll}
0 & 0 & 1
\end{array}\right) \boldsymbol{Y}^{-1}(z)\left(\begin{array}{l}
1 \\
0 \\
0
\end{array}\right),
\end{aligned}
$$

check out the comments after 5.1).

Proof of Theorem 2.8. For $z \in \mathbb{C} \backslash\left(\mathcal{S}_{1}^{ \pm} \cup \mathcal{S}_{2}^{ \pm} \cup \mathcal{S}_{2}^{ \pm} \cup \mathcal{U}\right)$, away from the contours $\Gamma_{R}$ depicted on Figure 20, we have

$$
\begin{aligned}
\boldsymbol{Y}^{-1}(z)=\operatorname{diag}\left(e^{N\left(g_{1}(z)-\frac{2}{3} z^{3}\right)}, e^{N\left(g_{2}(z)+\frac{1}{3} z^{3}\right)}, e^{N\left(g_{3}(z)+\frac{1}{3} z^{3}\right)}\right) \boldsymbol{F}^{T}(z) \\
\times(\boldsymbol{I}+\mathcal{O}(1 / N)) \operatorname{diag}\left(e^{-N r_{1}}, e^{-N r_{2}}, e^{-N r_{3}}\right),
\end{aligned}
$$


where we have used 6.38), so that

$$
\begin{aligned}
& 2 \pi i A_{n, m}(z)=e^{N\left(g_{2}(z)+\frac{1}{3} z^{3}-r_{1}\right)}\left(\begin{array}{lll}
0 & 1 & 0
\end{array}\right) \boldsymbol{F}^{T}(z)(\boldsymbol{I}+\mathcal{O}(1 / N))\left(\begin{array}{l}
1 \\
0 \\
0
\end{array}\right) \\
& =f_{12}(z) e^{N\left(g_{2}(z)+\frac{1}{3} z^{3}-r_{1}\right)}(1+\mathcal{O}(1 / N)) .
\end{aligned}
$$

On the other hand, for $z \in \mathcal{U} \backslash\left(\mathcal{S}_{1}^{ \pm} \cup \mathcal{S}_{2}^{ \pm} \cup \mathcal{S}_{3}^{ \pm}\right)$,

$$
\begin{array}{r}
\boldsymbol{Y}^{-1}(z)=\left(\boldsymbol{I}-\boldsymbol{M}_{32}\right) \operatorname{diag}\left(e^{N\left(g_{1}(z)-\frac{2}{3} z^{3}\right)}, e^{N\left(g_{2}(z)+\frac{1}{3} z^{3}\right)}, e^{N\left(g_{3}(z)+\frac{1}{3} z^{3}\right)}\right) \boldsymbol{F}^{T}(z) \\
\times(\boldsymbol{I}+\mathcal{O}(1 / N)) \operatorname{diag}\left(e^{-N r_{1}}, e^{-N r_{2}}, e^{-N r_{3}}\right),
\end{array}
$$

from where

$$
\begin{aligned}
2 \pi i A_{n, m}(z) & =e^{N\left(g_{2}(z)+\frac{1}{3} z^{3}-r_{1}\right)}\left(\begin{array}{lll}
0 & 1 & 0) \boldsymbol{F}^{T}(z)(\boldsymbol{I}+\mathcal{O}(1 / N)) \\
& =f_{12}(z) e^{N\left(g_{2}(z)+\frac{1}{3} z^{3}-r_{1}\right)}(1+\mathcal{O}(1 / N)), \\
0 \\
0
\end{array}\right)
\end{aligned}
$$

which coincides with the formula in 8 8.6. Again, as $\mathcal{S}_{1}^{ \pm}, \mathcal{S}_{2}^{ \pm}, \mathcal{S}_{3}^{ \pm}$can be made arbitrarily close to $\Delta_{1}, \Delta_{2}$ and $\Delta_{3}$, we conclude Theorem 2.8 for $z \in \mathbb{C} \backslash\left(\Delta_{1} \cup \Delta_{2} \cup\right.$ $\left.\Delta_{3}\right)$.

The functions

$$
\frac{A_{n, m}(z)}{f_{11}(z)} e^{-N\left(g_{2}(z)+\frac{1}{3} z^{3}-r_{1}\right)}
$$

are holomorphic on compact subsets of $\mathbb{C} \backslash\left(\Delta_{1} \cup \Delta_{3}\right)$, and by (8.6), 8.8) and Montel's Theorem, they form a normal family. This proves that

$$
\lim _{N \rightarrow \infty} \frac{A_{n, m}(z)}{f_{11}(z)} e^{-N\left(g_{2}(z)+\frac{1}{3} z^{3}-r_{1}\right)}=1
$$

also on $\Delta_{2} \backslash \mathbb{R}$, concluding the proof of Theorem 2.8 .

Proof of Theorem 2.4 for $A_{n, m}$. By construction $f_{12}$ does not vanish in $\mathbb{C} \backslash\left(\Delta_{1} \cup \Delta_{3}\right)$, so by Theorem 2.8 we conclude that all zeros of $A_{n, m}$ asymptotically belong to $\Delta_{1} \cup \Delta_{3}$, and

$$
\lim _{N \rightarrow \infty}\left|A_{n, m}(z)\right|^{1 / N}=e^{-\operatorname{Re}\left(-g_{2}(z)-\frac{1}{3} z^{3}+r_{1}\right)}
$$

locally uniformly on any compact subset of $\mathbb{C} \backslash\left(\Delta_{1} \cup \Delta_{3}\right)$. But by definition 3.19) and asymptotics 3.22,

$$
-g_{2}^{\prime}(z)-z^{2}=-\xi_{2}(z)-z^{2}=-\frac{\alpha}{z}+\mathcal{O}\left(1 / z^{2}\right), \quad z \rightarrow \infty .
$$

On the other hand, by $3.9-3.10$ and the definition of $\mu_{j}$ 's in 3.18,

$$
-g_{2+}^{\prime}(z)+g_{2-}^{\prime}(z)=\xi_{2-}(z)-\xi_{2+}(z)= \begin{cases}2 \pi i \mu_{1}^{\prime}(z), & \text { if } z \in \Delta_{1}, \\ 2 \pi i \mu_{3}^{\prime}(z), & \text { if } z \in \Delta_{3} \text { and } \tau>\tau_{c},\end{cases}
$$

from which

$$
-g_{2}^{\prime}(z)-z^{2}=C^{\mu_{1}+\mu_{3}}(z), \quad \mathbb{C} \backslash\left(\Delta_{1} \cup \Delta_{3}\right) .
$$


Thus, by the asymptotic formula 3.22 ,

$$
\operatorname{Re}\left(-g_{2}(z)-\frac{1}{3} z^{3}+r_{1}\right)=r_{1}-r_{2}+U^{\mu_{1}+\mu_{3}}(z), \quad \mathbb{C} \backslash\left(\Delta_{1} \cup \Delta_{3}\right),
$$

and

$$
\lim _{N \rightarrow \infty} \log \left|A_{n, m}(z)\right|^{1 / N}=r_{2}-r_{1}-U^{\mu_{1}+\mu_{3}}(z), \quad \mathbb{C} \backslash\left(\Delta_{1} \cup \Delta_{3}\right) .
$$

Since $\Delta_{1} \cup \Delta_{3}$ has empty interior and connected complement, this proves the statement of Theorem 2.4 for the weak limit of $\nu\left(A_{n, m}\right)$.

We now turn our attention to the asymptotic results for $B_{n, m}$, which require more care.

Proof of Theorem 2.9. For $z \in \mathbb{C} \backslash\left(\mathcal{S}_{1}^{ \pm} \cup \mathcal{S}_{2}^{ \pm} \cup \mathcal{S}_{3}^{ \pm} \cup \mathcal{U}\right)$, away from the contours $\Gamma_{R}$ depicted on Figure 20, we use (8.4) and (8.5) to arrive at

$$
\begin{aligned}
2 \pi i B_{n, m}(z) & =e^{N\left(g_{3}(z)+\frac{1}{3} z^{3}-r_{1}\right)}\left(\begin{array}{lll}
0 & 0 & 1
\end{array}\right) \boldsymbol{F}^{T}(z)(\boldsymbol{I}+\mathcal{O}(1 / N))\left(\begin{array}{l}
1 \\
0 \\
0
\end{array}\right) \\
& =f_{13}(z) e^{N\left(g_{3}(z)+\frac{1}{3} z^{3}-r_{1}\right)}(1+\mathcal{O}(1 / N)) .
\end{aligned}
$$

Recall that according to the construction at the beginning of Section 6.1. the contour $\gamma=\partial \mathcal{U}$ can be chosen arbitrarily within $\Omega_{+}$, so by 4.11 we can take it as close to $E_{\alpha} \cap \mathbb{H}_{-}$as we want. Also, the lenses $\mathcal{S}_{j}^{ \pm}$can be chosen arbitrarily close to $\Delta_{j}$. Thus, the asymptotic formula 8.9 is actually valid uniformly on compacts of $\mathbb{C} \backslash\left(\Delta_{1} \cup \Delta_{2} \cup \Delta_{3} \cup \Omega_{-}\right)$.

In a similar way, for $z \in \mathcal{U} \backslash\left(\mathcal{S}_{1}^{ \pm} \cup \mathcal{S}_{2}^{ \pm} \cup \mathcal{S}_{3}^{ \pm}\right)$we use (8.7) to get

$$
\begin{aligned}
2 \pi i B_{n, m}(z) & =\left(\begin{array}{lll}
0 & -1 & 1
\end{array}\right) \operatorname{diag}\left(e^{N\left(g_{1}(z)-\frac{2}{3} z^{3}\right)}, e^{N\left(g_{2}(z)+\frac{1}{3} z^{3}\right)}, e^{N\left(g_{3}(z)+\frac{1}{3} z^{3}\right)}\right) \\
& \times \boldsymbol{F}^{-1}(z)(\boldsymbol{I}+\mathcal{O}(1 / N)) \operatorname{diag}\left(e^{-N r_{1}}, e^{-N r_{2}}, e^{-N r_{3}}\right)\left(\begin{array}{c}
1 \\
0 \\
0
\end{array}\right) \\
& =\left(\begin{array}{lll}
0 & -e^{N\left(g_{2}(z)+\frac{1}{3} z^{3}-r_{1}\right)} & e^{N\left(g_{3}(z)+\frac{1}{3} z^{3}-r_{1}\right)}
\end{array}\right) \boldsymbol{F}^{T}(z)\left(\begin{array}{c}
1+\mathcal{O}(1 / N) \\
\mathcal{O}(1 / N) \\
\mathcal{O}(1 / N)
\end{array}\right)
\end{aligned}
$$

and thus,

$$
\begin{aligned}
2 \pi i B_{n, m}(z)= & f_{13}(z) e^{N\left(g_{3}(z)+\frac{1}{3} z^{3}-r_{1}\right)}(1+\mathcal{O}(1 / N)) \\
& -f_{12}(z) e^{N\left(g_{2}(z)+\frac{1}{3} z^{3}-r_{1}\right)}(1+\mathcal{O}(1 / N)),
\end{aligned}
$$

uniformly on compacts of $\mathcal{U} \backslash \Delta_{2}$

Obviously, which one of these two terms is dominant depends on whether we are in the domain $\Omega_{\alpha}$, see Definition 4.3 , or not. Indeed, we can summarize 8.9 and 8.10 by

$$
2 \pi i B_{n, m}(z)= \begin{cases}f_{13}(z) e^{N\left(g_{3}(z)+\frac{1}{3} z^{3}-r_{1}\right)}(1+\mathcal{O}(1 / N)), & z \in \mathbb{C} \backslash\left(\mathcal{S}_{j}^{ \pm} \cup \overline{\Omega_{\alpha}}\right), \\ -f_{12}(z) e^{N\left(g_{2}(z)+\frac{1}{3} z^{3}-r_{1}\right)}(1+\mathcal{O}(1 / N)), & z \in \Omega_{\alpha} \backslash \Delta_{2}=\Omega_{-}\end{cases}
$$

Due to analiticity of $g_{3}$ and $f_{13}$ across $\Delta_{1}$ (see (3.19) and (6.36), and of $g_{2}$ and $f_{12}$ across $\Delta_{2}$ (see 3.10 and $(6.36)$ ), we can extend the first of these formulas to 
compacts of $\mathbb{C} \backslash\left(\Omega_{\alpha} \cup \Delta_{2}\right)$, and the second of these formulas to the whole domain $\Omega_{\alpha}$, which proves the assertion of Theorem 2.9

Proof of Theorem 2.5. We turn now to Theorem 2.5.

With the definition of $H$ in Proposition 4.4, by 8.11 and Theorem 2.9 we conclude that

$$
\begin{aligned}
\lim _{N \rightarrow \infty} \log \left|B_{n, m}(z)\right|^{1 / N} & =H(z) \\
& =-U^{\mu_{B}}(z)+\operatorname{Re}\left(r_{3}-r_{1}\right), \quad z \in \mathbb{C} \backslash E_{\alpha},
\end{aligned}
$$

and this is enough to conclude Theorem 2.5

\section{Numerical EXPERIMENTS AND EMPIRICAL OBSERVATIONS}

Figures 16 are results of numerical experiments that we explain and further explore next.

The supports of the measures $\mu_{1}, \mu_{2}, \mu_{3}$ and $\mu_{B}$ coincide with critical trajectories of the canonical quadratic differential $\varpi$ in 4.1 . The numerical evaluation of these trajectories is explored and explained in our previous work [37, Appendix C].

To compute the zeros of the polynomials $A_{n, m}, B_{n, m}$ and $P_{n, m}$ we proceed as follows. Given the moments (2.1), we create the matrix of mixed moments

$$
H_{n, m}=\left(\begin{array}{cccccc}
\mathfrak{f}_{1}^{(0)} & \cdots & \mathfrak{f}_{1}^{(n-1)} & \mathfrak{f}_{2}^{(0)} & \cdots & \mathfrak{f}_{2}^{(m-1)} \\
\mathfrak{f}_{1}^{(1)} & \cdots & \mathfrak{f}_{1}^{(n)} & \mathfrak{f}_{2}^{(1)} & \cdots & \mathfrak{f}_{2}^{(m)} \\
& \vdots & & & \vdots & \\
\mathfrak{f}_{1}^{(m+n-1)} & \cdots & \mathfrak{f}_{1}^{(m+2 n-2)} & \mathfrak{f}_{2}^{(m+n-1)} & \cdots & \mathfrak{f}_{2}^{(2 m+n-2)}
\end{array}\right)
$$

We put the coefficients of $A_{n, m}$ and $B_{n, m}$ into column vectors $\vec{A}_{n, m}$ and $\vec{B}_{n, m}$ in increasing order. Then $\vec{A}_{n, m}$ and $\vec{B}_{n, m}$ solve the linear system 39 , Section 23.1]

$$
H_{n, m}\left(\begin{array}{l}
\vec{A}_{n, m} \\
\vec{B}_{n, m}
\end{array}\right)=\left(\begin{array}{c}
0 \\
\vdots \\
0 \\
1
\end{array}\right)
$$

Similarly, the coefficients of $P_{n, m}$ also solve a linear system, but with coefficients matrix given by the transpose of $H_{n, m}$.

Because the moments (2.1) are given explicitly, the linear system above can be solved numerically with high precision using any standard numerical solver. For our figures, we used the linear solver provided by Mathematica, working with 400 digits precision.

In Figures 2122 we display the zeros of $P_{n, m}, A_{n, m}$ and $B_{n, m}$ for the values $\alpha=1 / 5<\alpha_{c}, \alpha=3 / 10 \in\left(\alpha_{c}, \alpha_{2}\right)$ and $\alpha=3 / 8>\alpha_{2}$, and various choices of $n$ and $m$.

The same approach carries over to a more general situation: given an integer $K>2$, consider the type II monic multiple orthogonal polynomial $Q_{n, m}$ of degree 
$n+m$ defined through the orthogonality conditions

$$
\begin{aligned}
& \int_{\gamma_{\ell_{1} \cup \gamma_{\kappa_{1}}^{-}}} Q_{n, m}(z) z^{j} e^{-z^{K}} d z=0, \quad j=0, \ldots, n-1, \\
& \int_{\gamma_{\ell_{2}} \cup \gamma_{\kappa_{2}}^{-}} Q_{n, m}(z) z^{j} e^{-z^{K}} d z=0, \quad j=0, \ldots, m-1,
\end{aligned}
$$

where $\gamma_{\kappa}$ is the oriented ray from 0 to $\infty e^{2 \pi i \kappa / K}, \kappa=0, \ldots, K-1$, and $\gamma_{\kappa}^{-}$is the result of reversing the orientation of $\gamma_{\kappa}$.

Similarly, we define the type I MOPs $C_{n, m}$ and $D_{n, m}$ as polynomials of degrees at most $n-1$ and $m-1$, respectively, that satisfy

$$
\begin{aligned}
& \int_{\gamma_{\ell_{1}} \cup \gamma_{\kappa_{1}}^{-}} C_{n, m}(z) z^{j} e^{-z^{K}} d z+\int_{\gamma_{\ell_{2} \cup \gamma_{\kappa_{2}}^{-}}} D_{n, m}(z) z^{j} e^{-z^{K}} d z=0, \quad j \leq n+m-2, \\
& \int_{\gamma_{\ell_{1}} \cup \gamma_{\kappa_{1}}^{-}} C_{n, m}(z) z^{j} e^{-z^{K}} d z+\int_{\gamma_{\ell_{2}} \cup \gamma_{\kappa_{2}}^{-}} D_{n, m}(z) z^{j} e^{-z^{K}} d z=1, \quad j=n+m-1 .
\end{aligned}
$$

In the formulas above, we assume $\ell_{j} \neq \kappa_{j}, j=1,2$, and $\left\{\ell_{1}, \kappa_{1}\right\} \neq\left\{\ell_{2}, \kappa_{2}\right\}$ to ensure non-triviality. From

$$
\int_{0}^{\infty} x^{j} e^{-x^{K}} d x=\frac{1}{K} \Gamma\left(\frac{j+1}{K}\right),
$$

where $\Gamma(\cdot)$ is the Gamma function, and we can actually compute

$$
\int_{\gamma_{\ell} \cup \gamma_{\kappa}^{-}} Q_{n, m}(z) z^{j} e^{-z^{K}} d z=\left(e^{\frac{2 \pi i \ell(j+1)}{K}}-e^{\frac{2 \pi i \kappa(j+1)}{K}}\right) \frac{1}{K} \Gamma\left(\frac{j+1}{K}\right) .
$$

Using these moments and the linear system equivalent to (9.1), in Figures 2427 we plot the appropriately rescaled zeros of the polynomials $C_{n, m}, D_{n, m}$ and $Q_{n, m}$, for several values of $n$ and $m$, weights $e^{-z^{5}}$ and $e^{-z^{7}}$ and various choices of contours $\gamma_{l} \cup \gamma_{k}^{-}$. We devote the last paragraphs of this paper to some empirical discussion motivated by the results of these experiments.

According to Theorem 2.4. the limiting density of zeros for $A_{n, m}$ and $P_{n, m}$ coincide on $\Delta_{1}$. But Figures 21 22 allow us to conjecture that a stronger statement is true: for any $n, m$, the zeros of $A_{n, m}$ and $P_{n, m}$ on $\Delta_{1}$ interlace. This same interlacing appears for the real zeros of $Q_{n, m}$ and $C_{n, m}$ displayed in Figures 2425.

As one can see in Figures 21, 23, the zeros of $B_{n, m}$ are not on the real line. Nevertheless, they seem to display a "quasi-interlacing" pattern with the zeros of $P_{n, m}$ on the set where $\mu_{B}$ and $\mu_{2}$ coincide. A similar phenomenon can be observed in Figures 2427

The zeros of $P_{n, m}$ outside the real line seem to quickly approach on supp $\mu_{2}$, even for very small values of $n$ and $m$. The same is true for the zeros of $B_{n, m}$ on $\operatorname{supp} \mu_{B} \cap \operatorname{supp} \mu_{2}$, but its zeros on $\operatorname{supp} \mu_{B} \cap \mathbb{H}_{-}$seem to converge slower to their limiting support. Furthermore, the plots indicate that the measures $\mu_{3}$ and $\mu_{B}$ have very small mass near $a_{1}$ for $\alpha>\alpha_{c}$.

The contours of orthogonality in Figures 2425 were chosen in such a way that the polynomial $P_{n, m}$ is real and has some real zeros. As one can observe in these figures, for such choice of contours the zeros display some transitions similar to the ones rigorously described in this paper for the weight $e^{-z^{3}}$. 
In Figures 2427 indicate that for small values of $\alpha$ a portion of zeros of $P_{n, m}$ coincide, in the large degree limit, with the zeros of $C_{n, m}$, and the remaining zeros of $P_{n, m}$ coincide with the zeros of $B_{n, m}$. However, when $\alpha$ increases, zeros of $C_{n, m}$ and $D_{n, m}$ get closer to each other, and they seem to display some sort of repulsion. At this time, some of the zeros of $P_{n, m}$ do not follow zeros of $C_{n, m}$ or $D_{n, m}$ anymore.

\section{ACKNowledgements}

The first author was partially supported by the Spanish Government together with the European Regional Development Fund (ERDF) under grants MTM201453963-P and MTM2017-89941-P (from MINECO), by Junta de Andalucía (the Excellence Grant P11-FQM-7276 and the research group FQM-229), and by Campus de Excelencia Internacional del Mar (CEIMAR) of the University of Almería. 

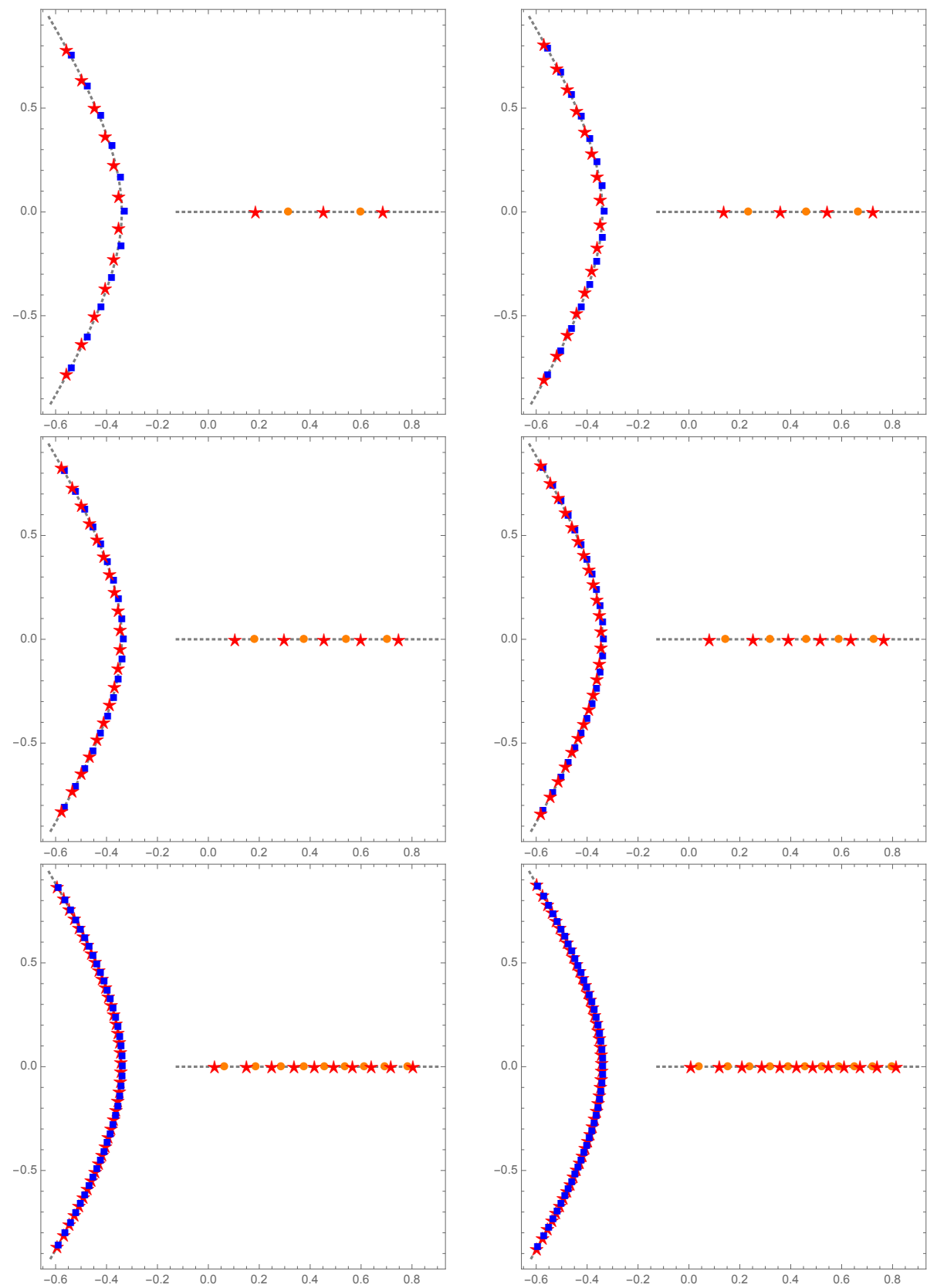

Figure 21. Zeros of $P_{n, m}$ (stars), $A_{n, m}$ (dots) and $B_{n, m}$ (squares), all corresponding to the same value $\alpha=1 / 5<$ $\alpha_{c}$. The dashed lines are the supports of $\mu_{1}$ and $\mu_{2}=$ $\mu_{B}$. From left to right, top to bottom: $(n, m)=$ $(3,12),(4,16),(5,20),(6,24),(10,24),(12,48)$. 

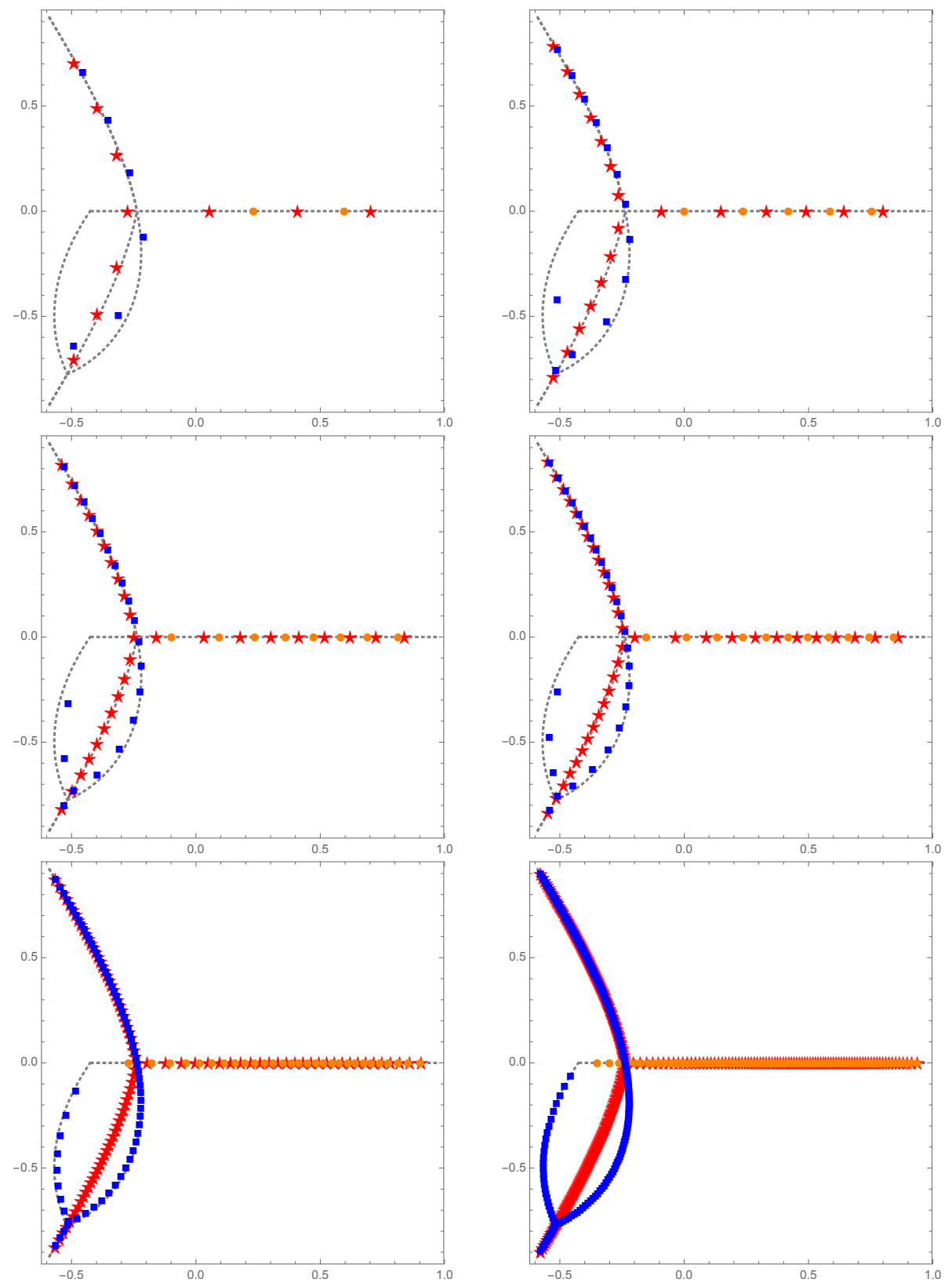

Figure 22. Zeros of $P_{n, m}$ (stars), $A_{n, m}$ (dots) and $B_{n, m}$ (squares), all corresponding to the same value $\alpha=3 / 10 \in$ $\left(\alpha_{c}, \alpha_{2}\right)$. The dashed lines are the supports of $\mu_{1}, \mu_{2}, \mu_{3}$ and $\mu_{B}$. From left to right, top to bottom: $(n, m)=$ $(3,7),(6,14),(9,21),(12,28),(30,70),(90,210)$. 

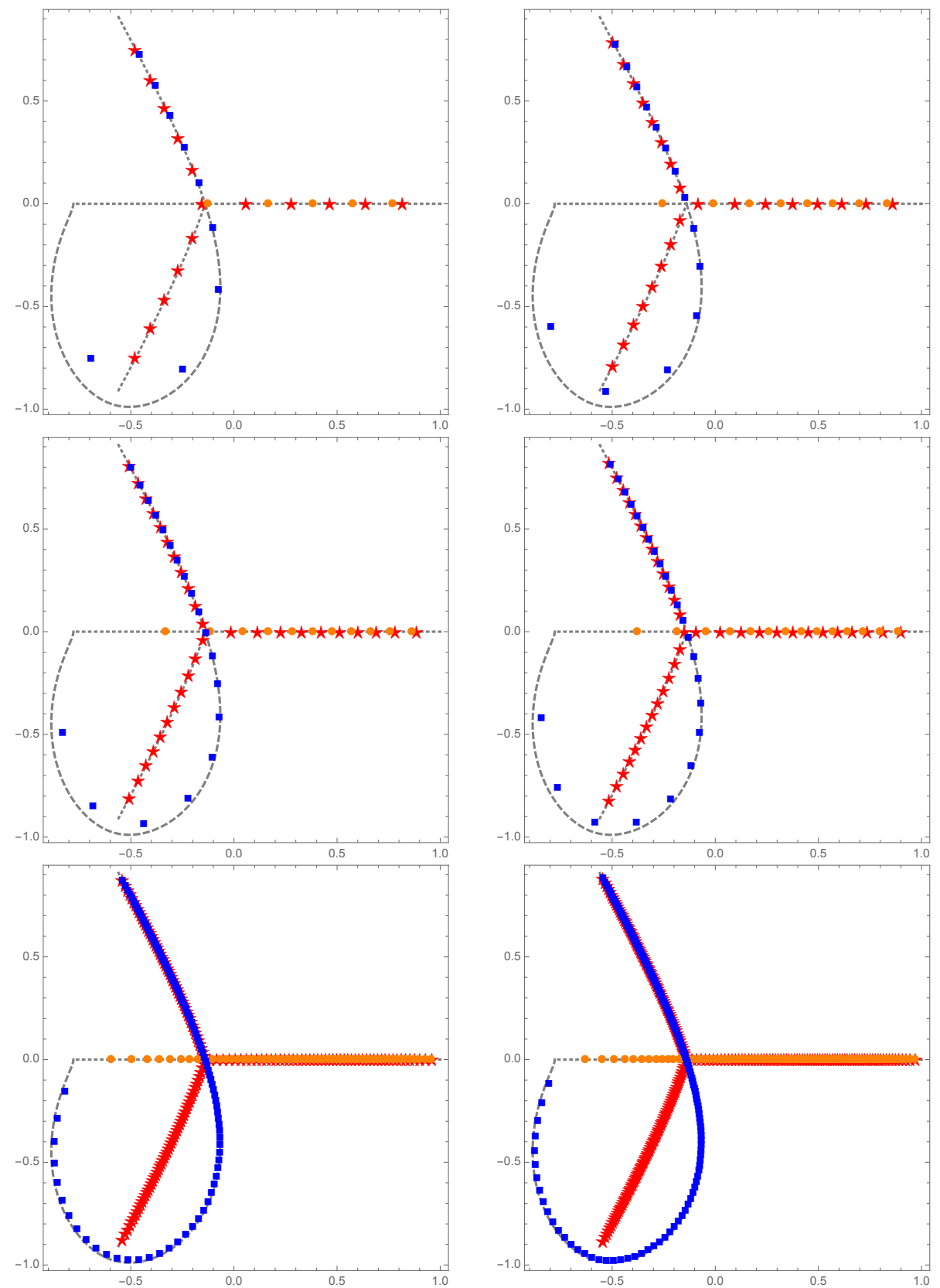

Figure 23. Zeros of $P_{n, m}$ (stars), $A_{n, m}$ (dots) and $B_{n, m}$ (squares), all corresponding to the same value $\alpha=3 / 8>$ $\alpha_{2}$. The dashed lines are the supports of $\mu_{1}, \mu_{2}, \mu_{3}$ and $\mu_{B}$. From left to right, top to bottom: $(n, m)=$ $(6,10),(9,15),(12,20),(15,25),(60,100),(90,150)$. 

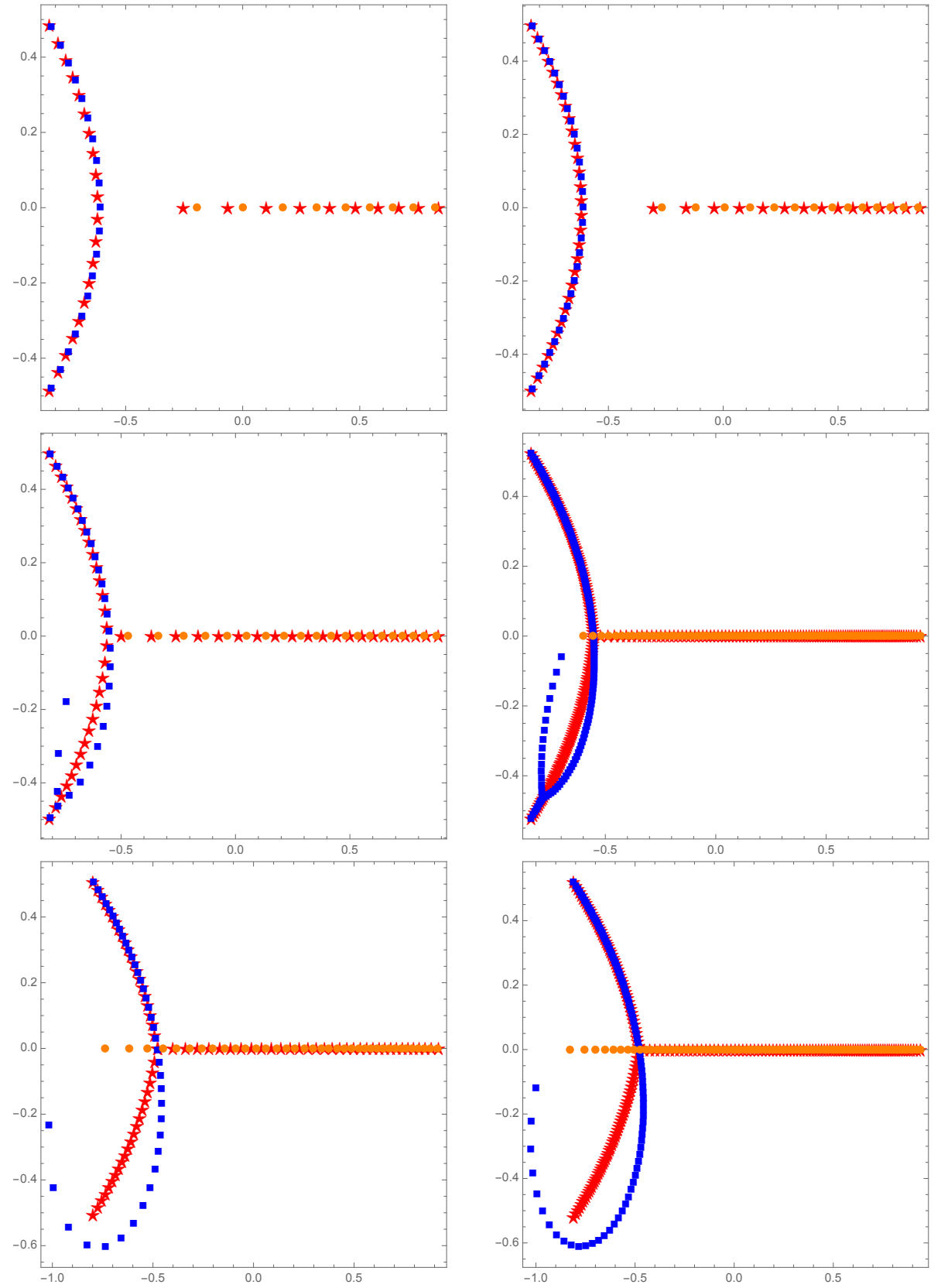

Figure 24. Rescaled zeros of $Q_{n, m}$ (stars), $C_{n, m}$ (dots) and $D_{n, m}$ (squares) when the weight is $e^{-z^{5}}$. In all the cases the orthogonality contour for $n$ is the union of rays $\left(e^{-\frac{4 \pi i}{5}} \infty, 0\right] \cup[0,+\infty)$, and for $m$ is the union $\left(e^{-\frac{4 \pi i}{5}} \infty, 0\right] \cup\left[0, e^{-\frac{4 \pi i}{5}} \infty\right)$. From left to right, top to bottom: $(n, m)=(10,20)$ and $(15,30)(\alpha=1 / 3) ;(n, m)=(21,30)$ and $(105,150)(\alpha=7 / 17) ;(n, m)=(38,40)$ and $(95,100)(\alpha=$ $19 / 39)$. 
56

A. MARTÍNEZ-FINKELSHTEIN AND G. SILVA
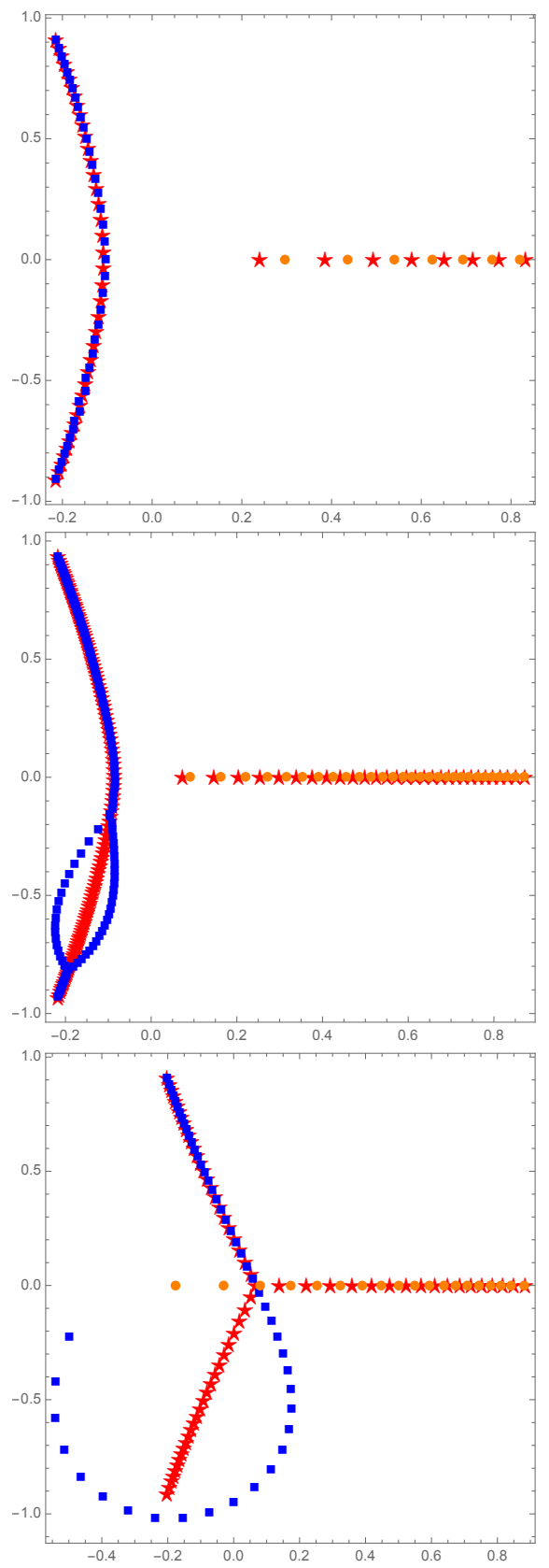
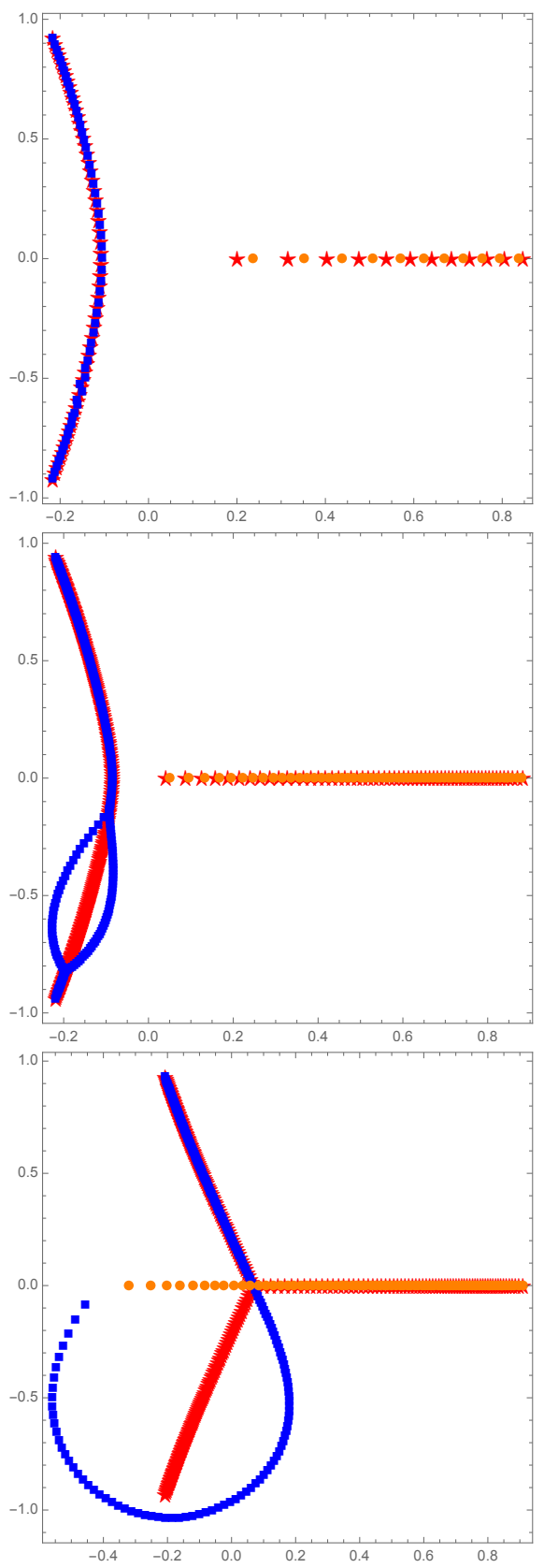

Figure 25. Rescaled zeros of $Q_{n, m}$ (stars), $C_{n, m}$ (dots) and $D_{n, m}$ (squares) when the weight is $e^{-z^{5}}$. In all the cases the orthogonality contour for $n$ is the union of rays $\left(e^{-\frac{4 \pi i}{7}} \infty, 0\right] \cup[0,+\infty)$, and for $m$ is the union $\left(e^{-\frac{4 \pi i}{7}} \infty, 0\right] \cup\left[0, e^{-\frac{4 \pi i}{7}} \infty\right)$. From left to right, top to bottom: $(n, m)=(8,40)$ and $(12,60)(\alpha=1 / 6) ;(n, m)=$ $(30,130)$ and $(60,260)(\alpha=3 / 16) ;(n, m)=(20,50)$ and $(80,200)$ $(\alpha=1 / 4)$. 

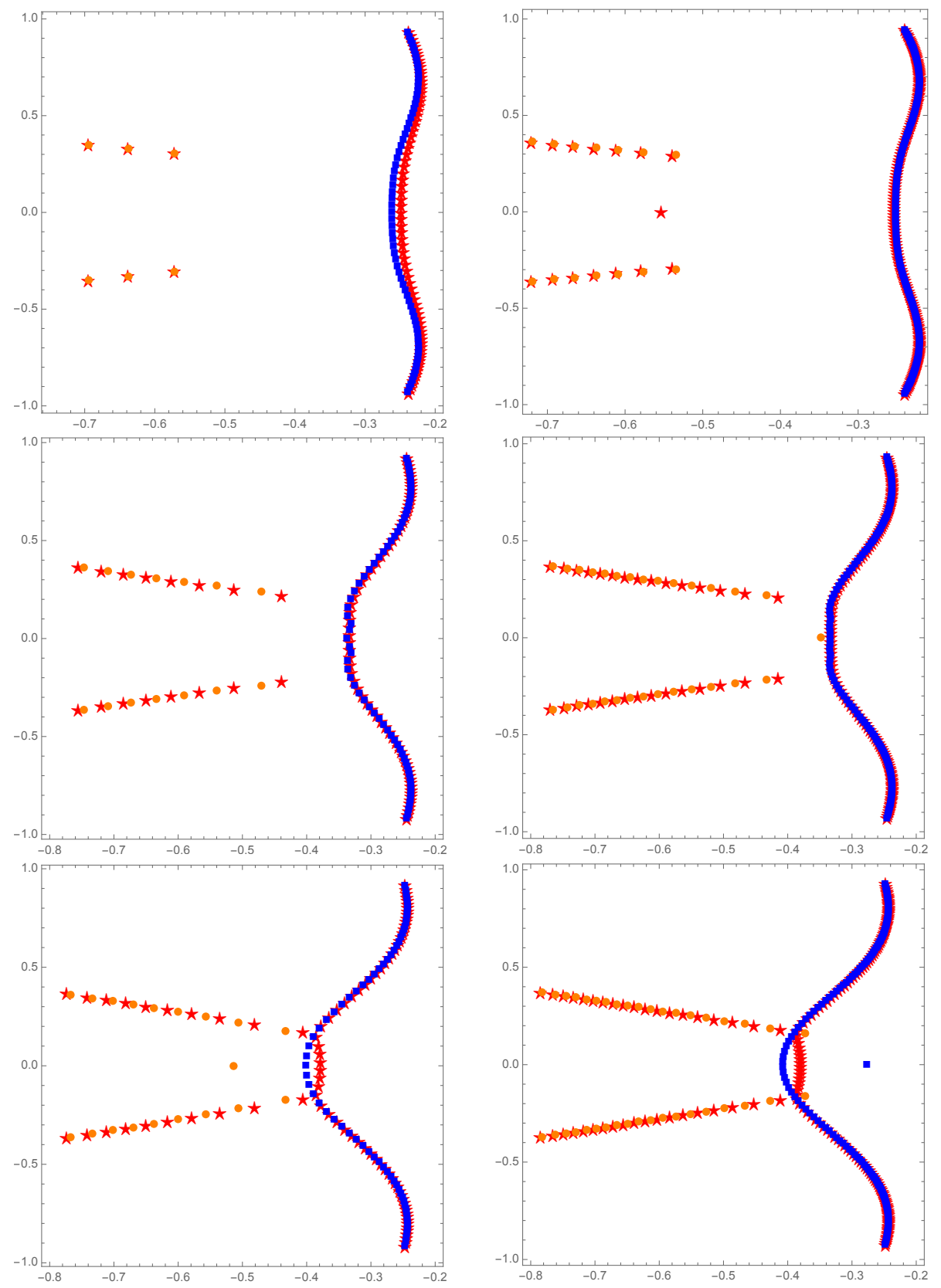

Figure 26. Rescaled zeros of $Q_{n, m}$ (stars), $C_{n, m}$ (dots) and $D_{n, m}$ (squares) when the weight is $e^{-z^{7}}$. In all the cases the orthogonality contour for $n$ is the union of rays $\left(e^{-\frac{6 \pi i}{7}} \infty, 0\right] \cup\left[0, e^{\frac{6 \pi i}{7}} \infty\right)$, and for $m$ is the union $\left(e^{-\frac{4 \pi i}{7}} \infty, 0\right] \cup\left[0, e^{\frac{4 \pi i}{7}} \infty\right)$. From left to right, top to bottom: $(n, m)=(7,77)$ and $(15,165)(\alpha=1 / 12) ;(n, m)=$ $(16,72)$ and $(30,135)(\alpha=2 / 11) ;(n, m)=(20,70)$ and $(40,140)$ $(\alpha=2 / 9)$. 

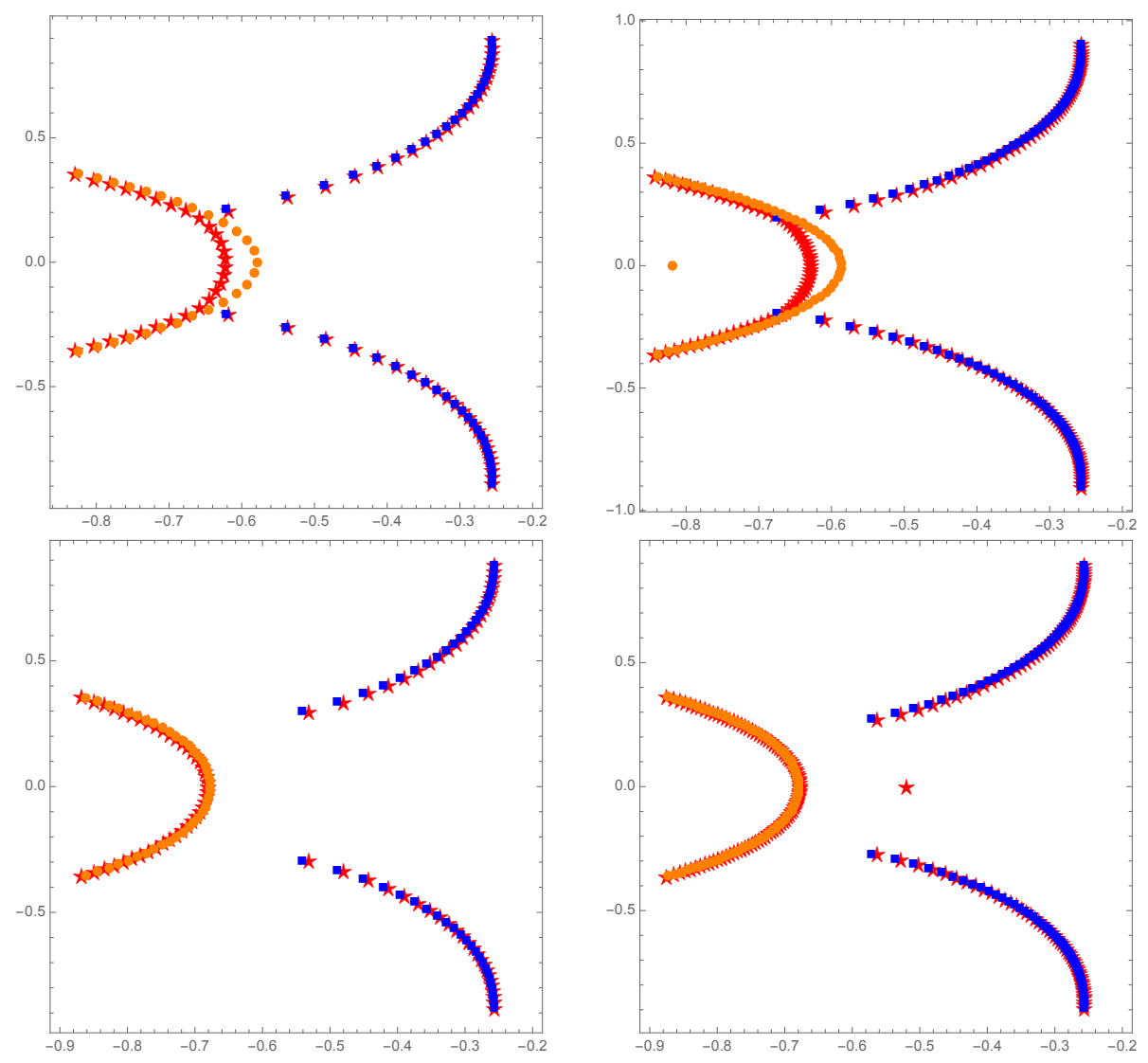

FiguRE 27. Rescaled zeros of $Q_{n, m}$ (stars), $C_{n, m}$ (dots) and $D_{n, m}$ (squares) when the weight is $e^{-z^{7}}$. In all the cases the orthogonality contour for $n$ is the union of rays $\left(e^{-\frac{6 \pi i}{7}} \infty, 0\right] \cup\left[0, e^{\frac{6 \pi i}{7}} \infty\right)$, and for $m$ is the union $\left(e^{-\frac{4 \pi i}{7}} \infty, 0\right] \cup\left[0, e^{\frac{4 \pi i}{7}} \infty\right)$. From left to right, top to bottom: $(n, m)=(28,49)$ and $(60,105)(\alpha=4 / 11) ;(n, m)=$ $(42,49)$ and $(90,105)(\alpha=6 / 13)$. 


\section{REFERENCES}

[1] G. Álvarez, L. Martínez-Alonso and E. Medina Determination of S-curves with applications to the theory of non-Hermitian orthogonal polynomials, J. Stat. Mech. Theory Exp. (2013), no. 6, P06006, 28.

[2] G. Álvarez, L. Martínez-Alonso and E. Medina Partition functions and the continuum limit in Penner matrix models, J. Phys. A 47 (2014), no. 31, 315205, 29.

[3] A. I. Aptekarev, Asymptotics of Hermite-Padé approximants for a pair of functions with branch points, Dokl. Akad. Nauk 422 (2008), no. 4, 443-445.

[4] A. I. Aptekarev, V. I. Buslaev, A. Martínez-Finkelshtein and S. P. Suetin, Padé approximants, continued fractions, and orthogonal polynomials, Uspekhi Mat. Nauk 66 (2011), no. 6(402), 37-122, translation in Russian Math. Surveys 66 (2011), no. 6, 1049-1131.

[5] A. I. Aptekarev and A. B. È. Kolèlaars, Hermite-Padé approximations and ensembles of multiple orthogonal polynomials, Uspekhi Mat. Nauk 66 (2011), no. 6(402), 123-190.

[6] A. I. Aptekarev, A. B. J. Kuijlaars and W. Van Assche, Asymptotics of Hermite-Padé rational approximants for two analytic functions with separated pairs of branch points (case of genus 0), Int. Math. Res. Pap. IMRP (2008), Art. ID rpm007, 128.

[7] A. I. Aptekarev, V. G. Lysov and D. N. Tulyakov, Random matrices with an external source and the asymptotics of multiple orthogonal polynomials, Mat. Sb. (2011), no. 2(202), 3-56.

[8] A. I. Aptekarev, V. G. Lysov and D. N. Tulyakov, The global eigenvalue distribution regime of random matrices with an anharmonic potential and an external source, Teoret. Mat. Fiz. (2009), no. 1(159), 34-57.

[9] A. I. Aptekarev, W. Van Assche and M. L. Yatsselev, Hermite-Padé approximants for a pair of Cauchy transforms with overlapping symmetric supports, Commun. Pure Appl. Math. 70 (2017), no. 3, 444-510.

[10] M. Bertola, M. Gekhtman and J. Szmigielski, Strong asymptotics for Cauchy biorthogonal polynomials with application to the Cauchy two-matrix model, J. Math. Phys. 54 (2013), no. $4,043517,25$.

[11] P. M. Bleher, S. Delvaux and A. B. J. Kuijlaars, Random matrix model with external source and a constrained vector equilibrium problem, Comm. Pure Appl. Math. 64 (2011), no. 1, $116-160$.

[12] P. M. Bleher and A. B. J. Kuijlaars, Large $n$ limit of Gaussian random matrices with external source.I, Comm. Math. Phys. 252 (2004), no. 1-3, 43-76.

[13] P. M. Bleher and A. B. J. Kuijlaars, Random matrices with external source and multiple orthogonal polynomials, Int. Math. Res. Not. (2004), no. 3, 109-129.

[14] P. M. Bleher and A. B. J. Kuijlaars, Orthogonal polynomials in the normal matrix model with a cubic potential, Adv. Math. 230 (2012), no. 3, 1272-1321.

[15] A. Deaño, D. Huybrechs and A. B. J. Kuijlaars, Asymptotic zero distribution of complex orthogonal polynomials associated with Gaussian quadrature, J. Approx. Theory 162 (2010), no. $12,2202-2224$.

[16] M. Duits, D. Geudens and A. B. J. Kuijlaars, A vector equilibrium problem for the two-matrix model in the quartic/quadratic case, Nonlinearity 24 (2011), no. 3, 951-993.

[17] M. Duits and A. B. J. Kuijlaars, Universality in the two-matrix model: a Riemann-Hilbert steepest-descent analysis, Comm. Pure Appl. Math. 62 (2009), no. 8, 1076-1153.

[18] M. Duits, A. B. J. Kuijlaars and M. Y. Mo, The Hermitian two matrix model with an even quartic potential, Mem. Amer. Math. Soc. 217 (2012), no. 1022, v+105.

[19] U. Fidalgo Prieto and G. López Lagomasino, Nikishin systems are perfect, Constr. Approx. 34 (2011), no. 3, 297-356.

[20] G. Filipuk, W. Van Assche and L. Zhang, Multiple orthogonal polynomials associated with an exponential cubic weight, J. Approx. Theory 190 (2015), 1-37.

[21] J. S. Geronimo, A. B. J. Kuijlaars and W. Van Assche, Riemann-Hilbert problems for multiple orthogonal polynomials, Special functions 2000: current perspective and future directions (Tempe, AZ), NATO Sci. Ser. II Math. Phys. Chem., vol. 30, Kluwer Acad. Publ., Dordrecht, 2001, pp. 23-59.

[22] A. A. Gonchar and E. A. Rakhmanov, On the convergence of simultaneous Padé approximants for systems of functions of Markov type, Trudy Mat. Inst. Steklov. 157 (1981), 31-48, 234, Number theory, mathematical analysis and their applications. 
[23] A. A. Gonchar and E. A. Rakhmanov, The equilibrium problem for vector potentials, Uspekhi Mat. Nauk 40 (1985), no. 4(244), 155-156.

[24] A. A. Gonchar and E. A. Rakhmanov, Equilibrium distributions and the rate of rational approximation of analytic functions, Mat. Sb. (N.S.) 134(176) (1987), no. 3, 306-352, 447.

[25] Ch. Hermite, Sur la fonction exponentielle, C. R. Acad. Sci. Paris 77 (1873), 18-24, 74-79, 226-233, 285-293.

[26] N. R. Ikonomov, R. K. Kovacheva, and S. P. Suetin, On the limit zero distribution of type I Hermite-Padé polynomials, preprint arXiv:1506.08031

[27] N. R. Ikonomov, R. K. Kovacheva, and S. P. Suetin, Zero Distribution of Hermite-Padé polynomials and convergence properties of Hermite approximants for multivalued analytic functions, preprint arXiv:1603.03314

[28] A. B. J. Kuijlaars, A. Martínez-Finkelshtein and F. Wielonsky, Non-intersecting squared Bessel paths and multiple orthogonal polynomials for modified Bessel weights, Comm. Math. Phys. 286 (2009), no. 1, 217-275.

[29] A. B. J. Kuijlaars, W. Van Assche and F. Wielonsky, Quadratic Hermite-Padé approximation to the exponential function: a Riemann-Hilbert approach, Constr. Approx. 21 (2005), no. 3, $351-412$.

[30] A. B. J. Kuijlaars and A. López-García, The normal matrix model with a monomial potential, a vector equilibrium problem, and multiple orthogonal polynomials on a star, Nonlinearity 28 (2015), no. 2, 347-406.

[31] A. B. J. Kuijlaars and M. Y. Mo, The global parametrix in the Riemann-Hilbert steepets descent analysis for orthogonal polynomials, Comput. Methdos Funct. Theory 11 (2011), no. $1,161-178$.

[32] A. B. J. Kuijlaars and G. L. F. Silva, S-curves in polynomial external fields, J. Approx. Theory 191 (2015), 1-37.

[33] M. Leurs and W. Van Assche, Jacobi-Angelesco multiple orthogonal polynomials on an r-star, preprint arXiv:1804.07512

[34] A. Markoff, Deux démonstrations de la convergence de certaines fractions continues, Acta Math. 19:1 (1895), 93-104.

[35] A. Martínez-Finkelshtein and E. A. Rakhmanov, Critical measures, quadratic differentials, and weak limits of zerosof Stieltjes polynomials, Comm. Math. Phys. 302 (2011), no. 1, $53-111$.

[36] A. Martínez-Finkelshtein and E. B. Saff, Asymptotic properties of Heine-Stieltjes and Van Vleck polynomials, J. Approx. Theory 118 (2002), no. 1, 131-151.

[37] A. Martínez-Finkelshtein and G. L. F. Silva, Critical measures for vector energy: Global structure of trajectories of quadratic differentials, Adv. Math. 302 (2016), 1137-1232.

[38] A. Martínez-Finkelshtein and E. A. Rakhmanov, On asymptotic behavior of Heine-Stieltjes and Van Vleck polynomials, Recent trends in orthogonal polynomials and approximation theory, Contemp. Math., vol. 507, Amer. Math. Soc., Providence, RI, 2010, pp. 209-232.

[39] I. Mourad, Classical and Quantum Orthogonal Polynomials in One Variable, Encyclopedia of Mathematics and its Applications, vol. 98, Cambridge University Press, Cambridge, UK, 2005.

[40] E. M. Nikishin and V. N. Sorokin, Rational approximations and orthogonality, Translations of Mathematical Monographs, vol. 92, American Mathematical Society, Providence, RI, 1991, Translated from the Russian by R. P. Boas.

[41] E. A. Rakhmanov, On the asymptotics of Hermite-Padé polynomials for two Markov functions, Mat. Sb. 202 (2011), no. 1, 133-140.

[42] E. A. Rakhmanov, Orthogonal polynomials and S-curves, Contemp. Math., vol. 578, Amer. Math. Soc., Providence, RI, 2012.

[43] E. A. Rakhmanov, The asymptotics of Hermite-Padé polynomials for two Markov-type functions, Sbornik: Mathematics 202 (2011), no. 1, 127.

[44] E. B. Saff and V. Totik, Logarithmic potentials with external fields, Springer-Verlag, Berlin, 1997.

[45] H. Stahl, Orthogonal polynomials with complex-valued weight function. I,II, Constr. Approx. 2 (1986), no. 3, 225-240, 241-251.

[46] H. Stahl, Orthogonal polynomials with respect to complex-valued measures, Orthogonal polynomials and their applications (Erice, 1990), IMACS Ann. Comput. Appl. Math., vol. 9, Baltzer, Basel, 1991, pp. 139-154. 
(AMF) Department of Mathematics, Baylor University, TX, USA, and Departamento de Matemáticas, Universidad de Almería, Spain

E-mail address, AMF: A_Martinez-Finkelshtein@baylor.edu

E-mail address, AMF: andrei@ual.es

(GS) University of Michigan, Ann Arbor - Mi, USA

E-mail address: silvag@umich.edu 